University of Tennessee Health Science Center

UTHSC Digital Commons

$12-2007$

\title{
Cardiopulmonary Predicators of Dysfunctional Ventilator Weaning Response after Coronary Artery Bypass Graft
}

Annapoorna Mary

University of Tennessee Health Science Center

Follow this and additional works at: https://dc.uthsc.edu/dissertations

Part of the Cardiovascular Diseases Commons, Perioperative, Operating Room and Surgical Nursing Commons, and the Surgery Commons

\section{Recommended Citation}

Mary, Annapoorna, "Cardiopulmonary Predicators of Dysfunctional Ventilator Weaning Response after Coronary Artery Bypass Graft" (2007). Theses and Dissertations (ETD). Paper 334. http://dx.doi.org/ 10.21007/etd.cghs.2007.0016.

This Dissertation is brought to you for free and open access by the College of Graduate Health Sciences at UTHSC Digital Commons. It has been accepted for inclusion in Theses and Dissertations (ETD) by an authorized administrator of UTHSC Digital Commons. For more information, please contact jwelch30@uthsc.edu. 


\title{
Cardiopulmonary Predicators of Dysfunctional Ventilator Weaning Response after Coronary Artery Bypass Graft
}

\begin{abstract}
Although the majority of coronary artery bypass graft (CABG) surgery patients are extubated within 6 to 8 hours following surgery, $20 \%$ to $40 \%$ of patients remain intubated 12 hours after surgery due to dysfunctional ventilator weaning response (DVWR). DVWR associated with increased morbidity and mortality (30\% to $43 \%$ ) following CABG surgery. Finding significant antecedence to predict DVWR could help to identify and prevent the complications from DVWR after CABG surgery. Literature review revealed that there is an association between cardiopulmonary indicators (CPI) and DVWR after CABG surgery. Cardiopulmonary indicators are the selected hemodynamic parameters that have an association with DVWR. The association of CPI with DVWR may be used to predict DVWR. Therefore, this study set out to find a predictive model for DVWR using CPI and significant antecedence.
\end{abstract}

The purposes of this research study were to describe the characteristics of $\mathrm{CPI}$ among patients with normal ventilator weaning response (NVWR) and dysfunctional ventilator weaning response (DVWR) after coronary artery bypass graft (CABG) surgery, to find the differences in characteristics of cardiopulmonary indicators between patients with NVWR and DVWR after CABG surgery, and to build a prediction model for DVWR with significant antecedence.

A retrospective case control study with time series design was utilized. An inclusion criteria guided purposive sampling technique was used to recruit 300 subjects from a retrospective audit of electronic medical records of patients who underwent CABG surgery between May 2003 and February 2006. Among the 300 subjects, 100 subjects constituted the case group and 200 constituted the control group. This study utilized descriptive and inferential statistical analysis, which was performed through SAS programs including PROC UNIVARIATE, PROC FREQ, PROC GLM, PROC REG, PROC MIXED REPEATED MEASURE ANOVA, and PROC LOGISTIC.

The study included such demographic variables as age and sex and clinical variables COPD, CHF, renal failure, number of grafts, and BSA, which were used for the description of the study sample as well as included in the analysis as covariates. The outcome variables of this study were DVWR and NVWR. The independent variable of the study was CPI, which included heart rate (HR), mean arterial pressure (MAP), central venous pressure (CVP), cardiac output (CO), respiratory rate (RR), mixed venous oxygen saturation $\left(\mathrm{SVO}_{2}\right)$, oxygen saturation $\left(\mathrm{SPO}_{2}\right)$, pulmonary artery diastolic pressure (PAD) and pulmonary artery systolic pressure (PASP). An hourly time series measurement of selected CPI for 12 consecutive hours after CABG surgery was used to predict DVWR.

Findings revealed that several antecedence including COPD, CHF, MAP, RR, CO, PAD, and PASP were significantly associated with DVWR. In addition, findings revealed that the odds in favor of DVWR for patients with COPD were 5.466 times higher as compared to patients without COPD, holding all other variables constant. The odds in favor of DVWR for patients with CHF were 3.930 times higher than for patients without $\mathrm{CHF}$, holding all other variables constant. The odds in favor of DVWR for patients with decrease $10 \mathrm{~mm} / \mathrm{Hg}$ mean MAP were 1.915 times the probability of NVWR, holding all other variables constant. This implies that hypotension increases risk of developing DVWR after CABG surgery. The odds in favor of DVWR for patients with decrease 5 points of mean RR were 2.978 times the probability of NVWR, holding all other variables constant. This implies that patients with lower RR are at risk of developing DVWR after CABG surgery. The odds in favor of DVWR for patients with decrease in mean CO by 2 points were 1.943 times the probability of NVWR, holding all other variables constant. This implies that patients with low $\mathrm{CO}$ are at the risk of developing DVWR after CABG surgery. The odds in favor of DVWR for patients with increase in mean PAD by $5 \mathrm{~mm} / \mathrm{hg}$ were 3.640 times the probability of NVWR, 
holding all other variables constant. This implies that patients with high PAD pressure are at risk of developing DVWR after CABG surgery. The odds in favor of DVWR for patients with decrease in mean PASP by $10 \mathrm{~mm} / \mathrm{hg}$ were 3.053 times the probability of NVWR, holding all other variables constant. This implies that the patients with low PASP are at risk of developing DVWR after CABG surgery.

In conclusion, the results of this study revealed significant antecedence to predict DVWR after CABG surgery, including COPD, CHF, MAP, RR, CO, PAD, and PASP. Therefore, this study concluded that the above-mentioned significant antecedence may be used to predict DVWR after CABG surgery in critical care. The implications from the conclusion are that the weaning protocols after CABG surgery may be tailored using these significant predictors. In addition, the study findings imply that patients with a history of COPD and CHF have significant risk of developing DVWR after CABG surgery. Therefore, this researcher recommends that weaning criteria be developed considering the above risk factors for high risk patients.

\section{Document Type}

Dissertation

\section{Degree Name}

Doctor of Philosophy (PhD)

\section{Program}

Nursing

\section{Research Advisor}

Carol Lynn Thompson, PhD

\section{Keywords}

Dysfunctional Ventilator Weaning Response, Cardiopulmonary Predictors, Cardiopulmonary Indicators, Coronary Artery Bypass Graft

\section{Subject Categories}

Cardiovascular Diseases | Diseases | Medicine and Health Sciences | Nursing | Perioperative, Operating Room and Surgical Nursing | Surgery 


\title{
Cardiopulmonary Predicators of Dysfunctional Ventilator Weaning Response after Coronary Artery Bypass Graft
}

\author{
A Dissertation \\ Presented to \\ The Graduate Studies Council \\ The University of Tennessee \\ Health Science Center
}

\author{
In Partial Fulfillment \\ Of the Requirements for the Degree \\ Doctor of Philosophy \\ From The University of Tennessee
}

\author{
By \\ Annapoorna Mary \\ December 2007
}


Copyright (C) 2007 by Annapoorna Mary

All rights reserved 


\section{DEDICATION}

This dissertation is dedicated to the divine wisdom and the ever-loving presence of God in the most Holy Eucharistic Adoration of the Blessed Sacrament with a joyful heart for the gift of wisdom, knowledge, resources, strength, endurance, and success. 


\section{ACKNOWLEDGEMENTS}

I would like to thank my parents for the gift of life, education, guidance, support, sacrifices, and love that helped me pursue the career in nursing. I would like to express

my heart-felt gratitude to my husband, whose sacrifice and love motivated and supported my higher educational endeavors. I also thank my children, Noel and Joel, for their constant support and motivation that helped me to accomplish this research.

I would like to extend gratitude, in addition, to Dr. Carol Lynn Thompson. Dr. Thompson served as the dissertation chair for this research. In her role, Dr. Thompson demonstrated expert resourcefulness, kind mentorship, inspirational leadership, and student advocacy. I acknowledge the all-round development and transformation I underwent to become a researcher under her guidance.

I also thank dissertation committee members Dr. Veronica Engle, Dr. Ann Cashion, Dr. Wendy Likes, and Dr. Bryan Williams for their valuable contributions to this research through their expertise and support.

A very special thanks goes to Dr. Mona Wicks, Dr. Donna Hathaway, and Dr. Carolyn Graff for their guidance and expert direction. In addition, I express gratitude to all my teachers, mentors, and benefactors whose expert contributions transformed the researcher to accomplish this degree. I remember and salute all the architects of my academic achievements from grade school to the doctorate degree, including sisters of Seven Dollars (St. Antony's Middle School), Sr. Michelin (Holy Cross High School); Sr. Pepin (St. Thomas Hr. Secondary); Mother Elizabeth Ann, Sr. Emmanuel (St. Ann's School of Nursing); Dr. Roslind Convey; Dr. Jaya Mohan Raj; Dr. Rajeswari Vaithea 
Nathan (Madras Medical College), Mr. P. V. Ramachandran (Sri. Ramachandra Medical College \& Research Institute), Dr. Carol Thompson, Dr. Mona Wicks, Dr. Donna Hathaway, Dr. Veronica Engle, Dr. Ann Cashion, Dr. Wendy Likes, and Dr. Bryan Williams (University of Tennessee Health Science Center).

I would also like to thank the Mid-South hospital administration for their approval to conduct the study in the Mid-South - Memphis hospital. Lastly, I would like to acknowledge the support and cooperation rendered by the IRB and Health Information Management Systems Department of Mid-South Hospital, which was a critical contribution for the accomplishment of this research study.

I would like to extend my appreciation to Shirley Hancock and Dr. Mary McNealy and for their editorial guidance. 


\begin{abstract}
Although the majority of coronary artery bypass graft (CABG) surgery patients are extubated within 6 to 8 hours following surgery, $20 \%$ to $40 \%$ of patients remain intubated 12 hours after surgery due to dysfunctional ventilator weaning response (DVWR). DVWR associated with increased morbidity and mortality (30\% to $43 \%$ ) following CABG surgery. Finding significant antecedence to predict DVWR could help to identify and prevent the complications from DVWR after CABG surgery. Literature review revealed that there is an association between cardiopulmonary indicators (CPI) and DVWR after CABG surgery. Cardiopulmonary indicators are the selected hemodynamic parameters that have an association with DVWR. The association of CPI with DVWR may be used to predict DVWR. Therefore, this study set out to find a predictive model for DVWR using CPI and significant antecedence.

The purposes of this research study were to describe the characteristics of CPI among patients with normal ventilator weaning response (NVWR) and dysfunctional ventilator weaning response (DVWR) after coronary artery bypass graft (CABG) surgery, to find the differences in characteristics of cardiopulmonary indicators between patients with NVWR and DVWR after CABG surgery, and to build a prediction model for DVWR with significant antecedence.
\end{abstract}

A retrospective case control study with time series design was utilized. An inclusion criteria guided purposive sampling technique was used to recruit 300 subjects from a retrospective audit of electronic medical records of patients who underwent CABG surgery between May 2003 and February 2006. Among the 300 subjects, 100 
subjects constituted the case group and 200 constituted the control group. This study utilized descriptive and inferential statistical analysis, which was performed through SAS programs including PROC UNIVARIATE, PROC FREQ, PROC GLM, PROC REG, PROC MIXED REPEATED MEASURE ANOVA, and PROC LOGISTIC.

The study included such demographic variables as age and sex and clinical variables $\mathrm{COPD}, \mathrm{CHF}$, renal failure, number of grafts, and BSA, which were used for the description of the study sample as well as included in the analysis as covariates. The outcome variables of this study were DVWR and NVWR. The independent variable of the study was CPI, which included heart rate (HR), mean arterial pressure (MAP), central venous pressure (CVP), cardiac output (CO), respiratory rate (RR), mixed venous oxygen saturation $\left(\mathrm{SVO}_{2}\right)$, oxygen saturation $\left(\mathrm{SPO}_{2)}\right.$, pulmonary artery diastolic pressure (PAD) and pulmonary artery systolic pressure (PASP). An hourly time series measurement of selected CPI for 12 consecutive hours after CABG surgery was used to predict DVWR.

Findings revealed that several antecedence including COPD, CHF, MAP, RR, CO, PAD, and PASP were significantly associated with DVWR. In addition, findings revealed that the odds in favor of DVWR for patients with COPD were 5.466 times higher as compared to patients without COPD, holding all other variables constant. The odds in favor of DVWR for patients with CHF were 3.930 times higher than for patients without CHF, holding all other variables constant. The odds in favor of DVWR for patients with decrease $10 \mathrm{~mm} / \mathrm{Hg}$ mean MAP were 1.915 times the probability of NVWR, holding all other variables constant. This implies that hypotension increases risk of developing DVWR after CABG surgery. The odds in favor of DVWR for patients with decrease 5 points of mean RR were 2.978 times the probability of NVWR, holding all other 
variables constant. This implies that patients with lower RR are at risk of developing DVWR after CABG surgery. The odds in favor of DVWR for patients with decrease in mean CO by 2 points were 1.943 times the probability of NVWR, holding all other variables constant. This implies that patients with low $\mathrm{CO}$ are at the risk of developing DVWR after CABG surgery. The odds in favor of DVWR for patients with increase in mean PAD by $5 \mathrm{~mm} / \mathrm{hg}$ were 3.640 times the probability of NVWR, holding all other variables constant. This implies that patients with high PAD pressure are at risk of developing DVWR after CABG surgery. The odds in favor of DVWR for patients with decrease in mean PASP by $10 \mathrm{~mm} / \mathrm{hg}$ were 3.053 times the probability of NVWR, holding all other variables constant. This implies that the patients with low PASP are at risk of developing DVWR after CABG surgery.

In conclusion, the results of this study revealed significant antecedence to predict DVWR after CABG surgery, including COPD, CHF, MAP, RR, CO, PAD, and PASP. Therefore, this study concluded that the above-mentioned significant antecedence may be used to predict DVWR after CABG surgery in critical care. The implications from the conclusion are that the weaning protocols after CABG surgery may be tailored using these significant predictors. In addition, the study findings imply that patients with a history of COPD and CHF have significant risk of developing DVWR after CABG surgery. Therefore, this researcher recommends that weaning criteria be developed considering the above risk factors for high risk patients. 
TABLE OF CONTENTS

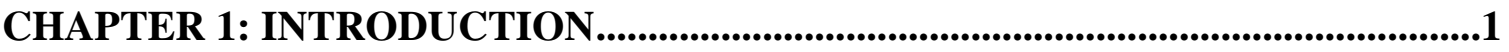

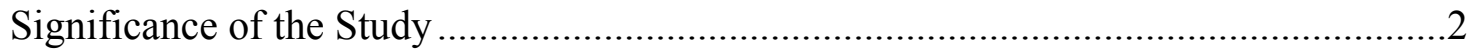

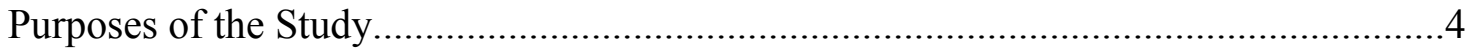

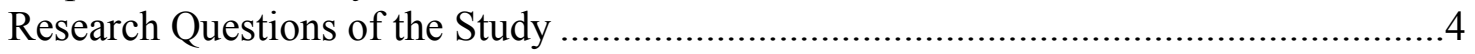

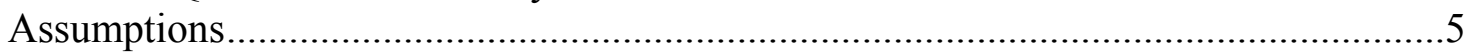

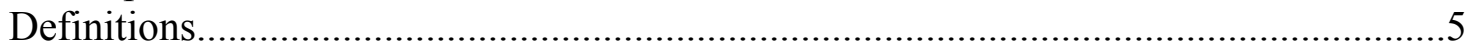

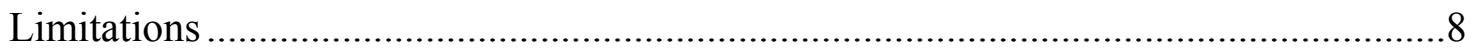

CHAPTER 2: REVIEW OF LITERATURE ...........................................................9

Definition of Dysfunctional Ventilator Weaning Response ...........................................9

Defining Characteristics of Dysfunctional Ventilator Weaning Response.....................10

Prevalence and Risk Factors .................................................................................10

Prediction in Prevention of Dysfunctional Ventilator Weaning Response.....................12

Cardiopulmonary Indicators in Prediction of Dysfunctional........................................14

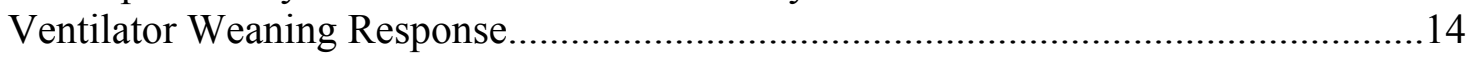

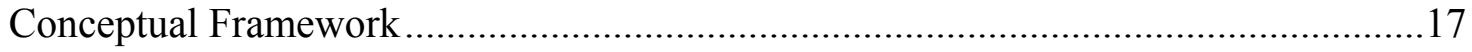

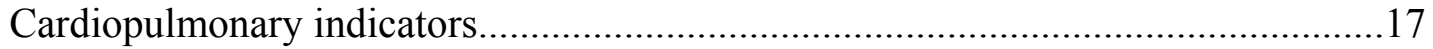

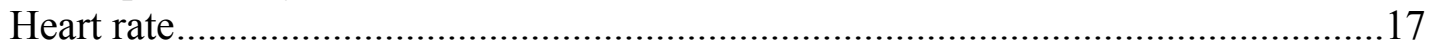

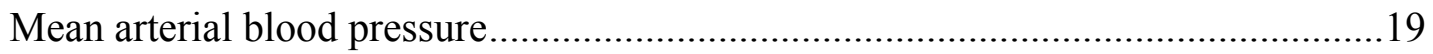

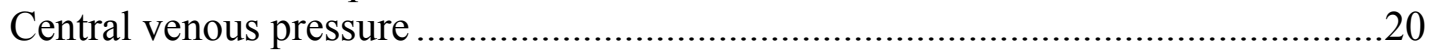

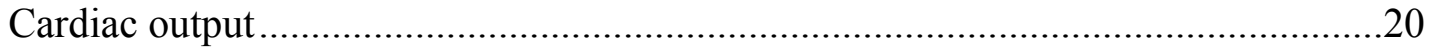

Mixed venous oxygen saturation ..................................................................21

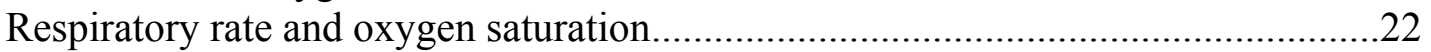

Pulmonary artery diastolic pressure and pulmonary artery systolic pressure............22

Effects of comorbidity in alteration of cardiopulmonary indicators...........................24

Cardiopulmonary monitoring............................................................................26

Dysfunctional ventilator weaning response ............................................................28

Perioperative risk factors of coronary artery bypass graft surgery associated with dysfunctional ventilator weaning response .............................................................28

Pathophysiology of dysfunctional ventilator weaning response..................................30

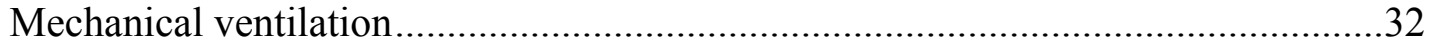

Weaning from mechanical ventilation...................................................................33

Conceptual model of predictive association of cardiopulmonary indicators with dysfunctional ventilator weaning response ..............................................................36

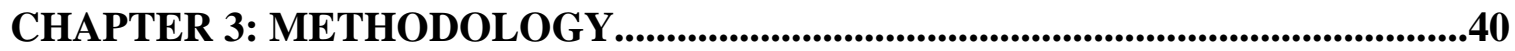

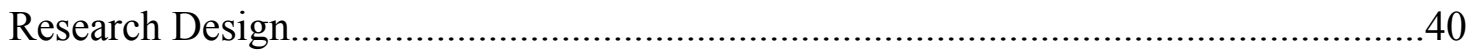

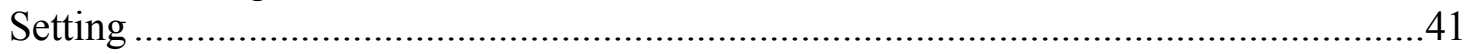

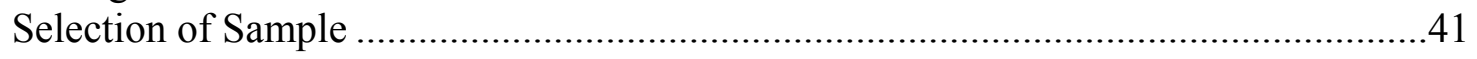

Selection of cases and controls ........................................................................42

Sample size and sampling technique ................................................................42

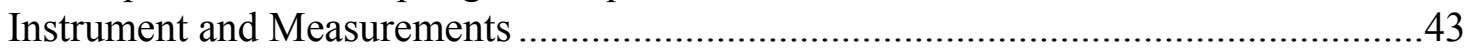




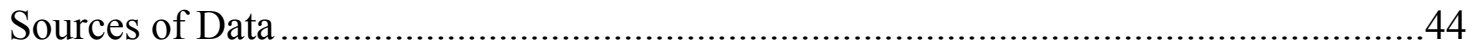

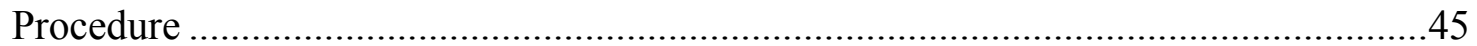

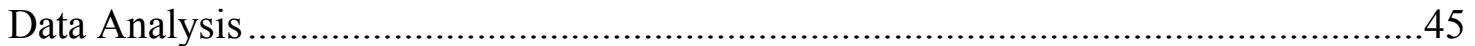

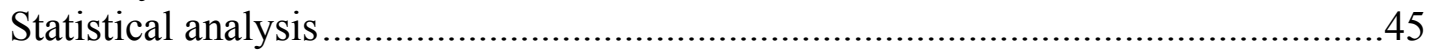

Testing overall model fitness ..............................................................................

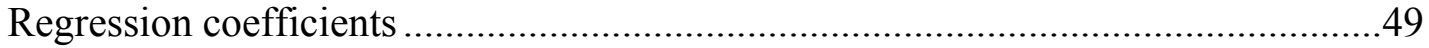

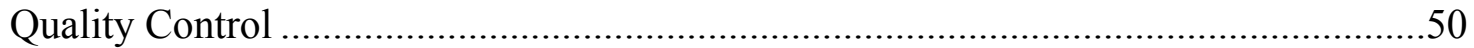

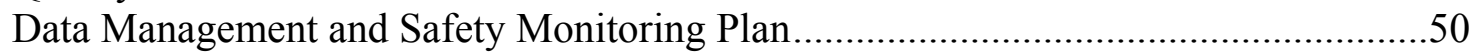

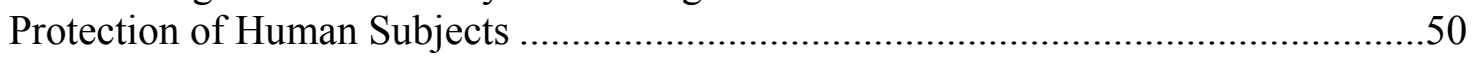

CHAPTER 4: RESULTS .........................................................................................52

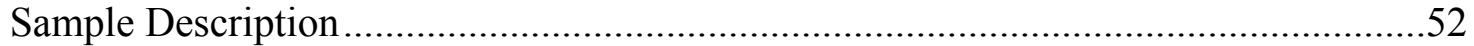

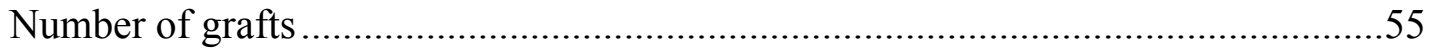

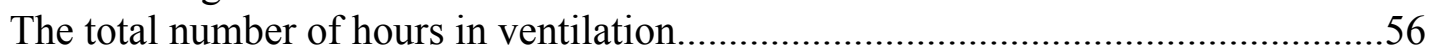

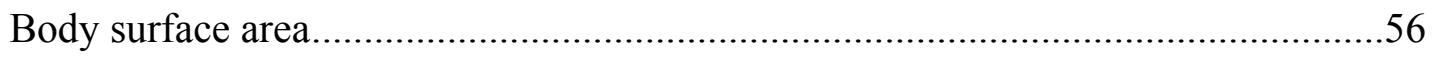

Post operative ventilator settings and medications ………....................................56

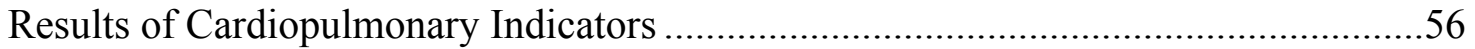

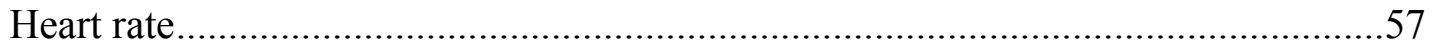

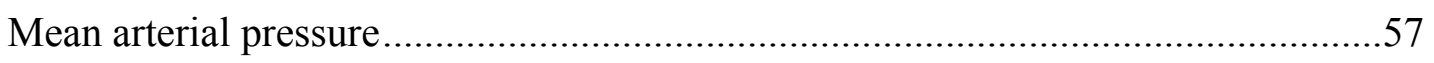

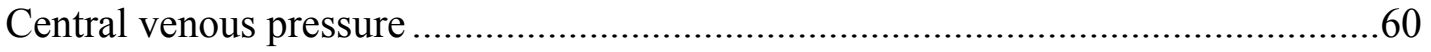

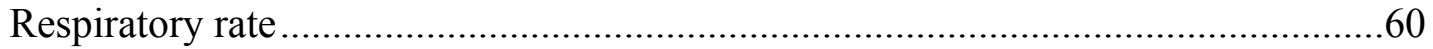

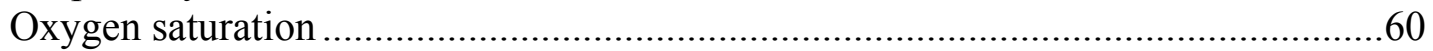

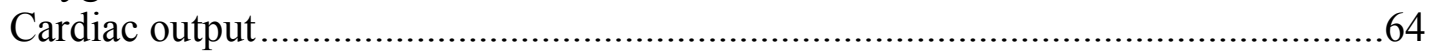

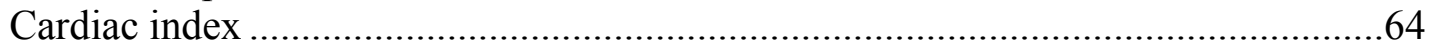

Pulmonary artery diastolic pressure ................................................................64

Pulmonary artery systolic pressure ……………………...................................68

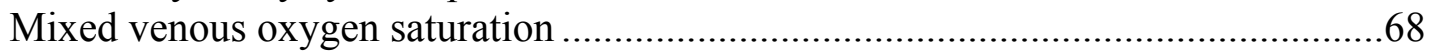

Differences in the Characteristics of Cardiopulmonary Indicators...............................71

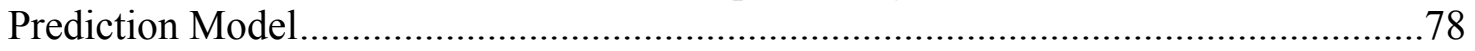

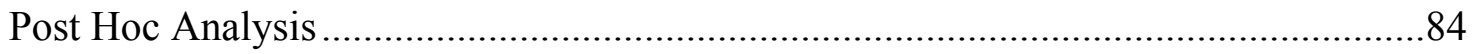

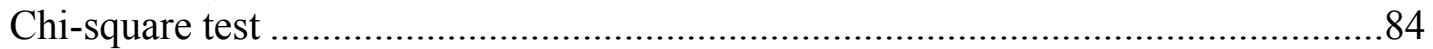

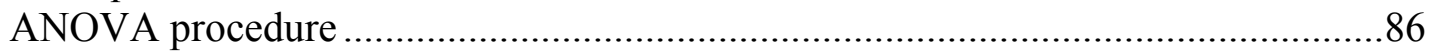

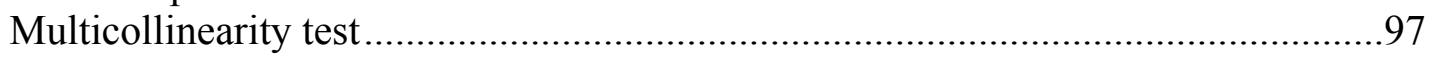

Logistic regression procedure ……………………….......................................99

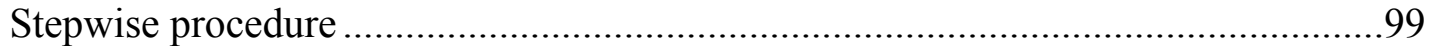

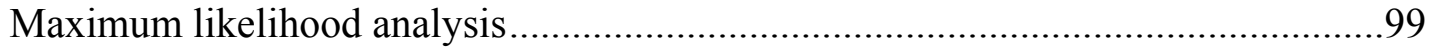

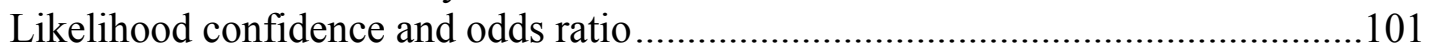

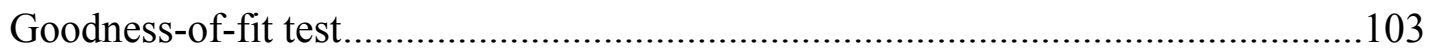

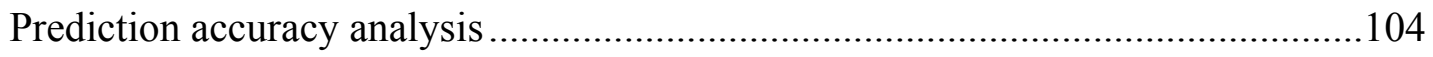

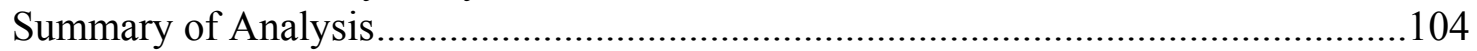

CHAPTER 5: CONCLUSION..........................................................................108

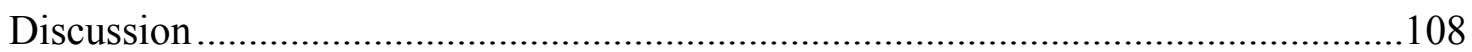

Discussion of Study Variables and Findings ...............................................................109

Cardiopulmonary Indicators and Dysfunctional Ventilator Weaning Response .........110

Heart rate and dysfunctional ventilator weaning response ......................................110 
Mean arterial pressure and dysfunctional ventilator weaning response

Central venous pressure and dysfunctional ventilator weaning response

Respiratory rate and dysfunctional ventilator weaning response

Oxygen saturation and dysfunctional ventilator weaning response........................114

Cardiac output dysfunctional ventilator weaning response

Cardiac index and dysfunctional ventilator weaning response

Pulmonary artery diastolic pressure and dysfunctional ventilator weaning response.

Pulmonary artery systolic pressure and dysfunctional ventilator weaning

response.

Mixed venous oxygen saturation and dysfunctional ventilator weaning response..119

Discussion of Demographic and Clinical Variables ....

Age and dysfunctional ventilator weaning response

Sex and dysfunctional ventilator weaning response.

Renal failure and dysfunctional ventilator weaning response.

Chronic obstructive pulmonary disease and dysfunctional ventilator weaning response.

Congestive heart failure and dysfunctional ventilator weaning response.

Number of grafts and dysfunctional ventilator weaning response.

Body surface area and dysfunctional ventilator weaning response

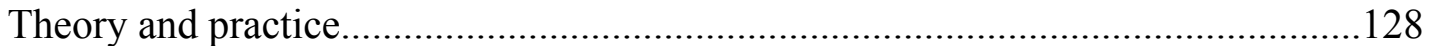

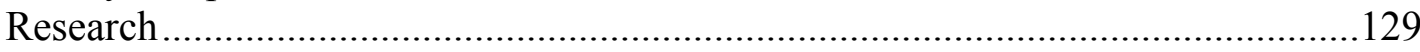

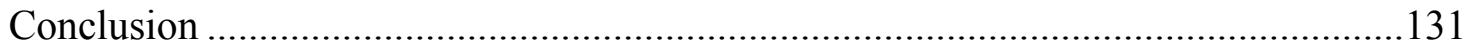

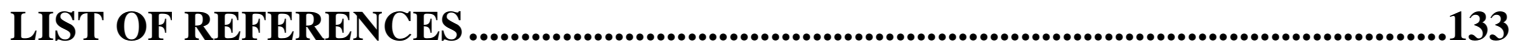

APPENDIX A: UNIVERSITY OF TENNESSEE HEALTH SCIENCE CENTER

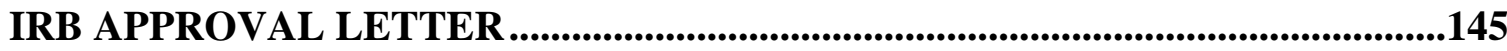

APPENDIX B: ST. FRANCIS HOSPITAL, MEMPHIS, IRB APPROVAL

VITA 


\section{LIST OF TABLES}

Table 4.1. Frequency and Percentage of Subjects in Demographic Categories. ........54

Table 4.2. Means and Standard Deviations of Heart Rate (HR) for Cases and

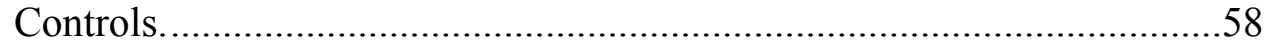

Table 4.3. Means and Standard Deviations of Mean Arterial Pressure for Cases and Controls....................................................................................59

Table 4.4. Means and Standard Deviations of Central Venous Pressure for Cases and Controls.......................................................................................61

Table 4.5. Means and Standard Deviations of Respiratory Rate for Cases and Controls.

Table 4.6. Means and Standard Deviations of Oxygen Saturation for Cases and Controls.....................................................................................6 63

Table 4.7. Means and Standard Deviations of Cardiac Output for Cases and Controls.....

Table 4.8. Means and Standard Deviations of Cardiac Index for Cases and Controls. .66

Table 4.9. Means and Standard Deviation Pulmonary Artery Diastolic Pressure for Cases and Controls.

Table 4.10. Means and Standard Deviations of Pulmonary Artery Systolic Pressure for Cases and Controls.

Table 4.11. Means and Standard Deviations of Mixed Venous Oxygen Saturation for Cases and Controls.............................................................................70

Table 4.12. Differences between Cases and Controls in Mean Heart Rate per Minute. .72

Table 4.13. Differences between Cases and Controls in Mean Arterial Pressure in

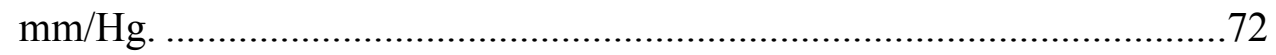

Table 4.14. Differences between Cases and Controls in Mean Central Venous

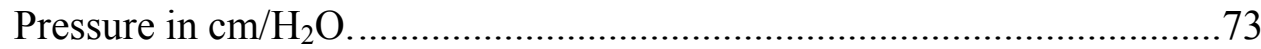

Table 4.15. Differences between Cases and Controls in Mean Respiratory Rate per

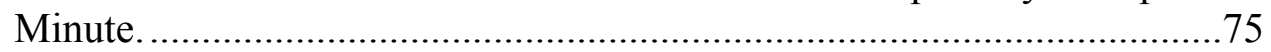

Table 4.16. Differences between Cases and Controls in Mean Oxygen Saturation

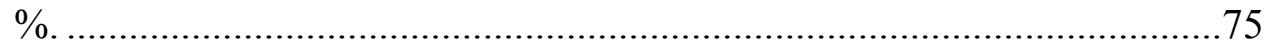

Table 4.17. Differences between Cases and Controls in Mean Cardiac Output Liters per Minute.........................................................................76

Table 4.18. Differences between Cases and Controls in Mean Cardiac Index Liters per Minute...................................................................................76

Table 4.19. Differences between Cases and Controls in Mean Pulmonary Artery Diastolic Pressure in $\mathrm{mm} / \mathrm{Hg}$..............................................................77

Table 4.20. Differences between Cases and Controls in Mean Pulmonary Artery

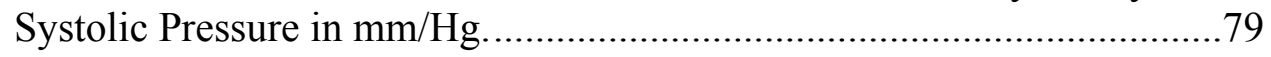

Table 4.21. Differences between Cases and Controls in Mean Mixed Venous Oxygen Saturation \%. .79

Table 4.22. Correlation Matrix for Cardio Pulmonary Indicators. .82 
Table 4.23. The Regression Model for Cardiopulmonary Indicators. .82

Table 4.24. Analysis of Maximum Likelihood Estimates of Cardiopulmonary Indicators. .83

Table 4.25. ANOVA Table for Heart Rate (HR) Beat per Minute Post Hoc. ..............89

Table 4.26. ANOVA Table for Mean Arterial Pressure (MAP) mm/Hg Post Hoc. .....89

Table 4.27. ANOVA Table for Central Venous Pressure (CVP) $\mathrm{cm} / \mathrm{H}_{2} \mathrm{O}$ Post Hoc....90

Table 4.28. ANOVA Table for Respiratory Rate (RR) per Minute Post Hoc..............92

Table 4.29. ANOVA Table for Oxygen Saturation $\left(\mathrm{SPO}_{2}\right) \%$ Post Hoc.......................92

Table 4.30. ANOVA Table for Cardiac Output (CO) Liter per Minute Post Hoc........93

Table 4.31. ANOVA Table for Cardiac Index (CI) Liter per Minute Post Hoc. ..........95

Table 4.32. ANOVA Table for Pulmonary Artery Diastolic Pressure (PAD) mm/Hg Post Hoc.............................................................................95

Table 4.33. ANOVA Table for Pulmonary Artery Systolic Pressure (PASP) $\mathrm{mm} / \mathrm{Hg}$ Post Hoc.

Table 4.34. ANOVA Table for Mixed Venous Oxygen Saturation $\left(\mathrm{SVO}_{2}\right) \%$ Post

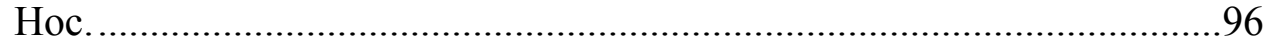

Table 4.35. Parameter Estimates of Regression Model for Dysfunctional Ventilator Weaning Response with Antecedence Post Hoc. . .98

Table 4.36. Analysis of Maximum Likelihood Estimates of Antecedence Post Hoc. 100 Table 4.37. Profile of Likelihood Confidence Interval for Adjusted Odds Ratios of Antecedence Post Hoc. .102 


\section{LIST OF FIGURES}

Figure 2.1. Proposed Conceptual Model of Cardiopulmonary Indicators in Predicting Dysfunctional Ventilator Weaning Response after CABG

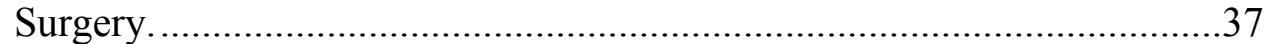

Figure 4.1. Mean Heart Rate Trend............................................................................58

Figure 4.2. Mean Arterial Pressure Trend..................................................................59

Figure 4.3. Mean Central Venous Pressure Trend. ....................................................61

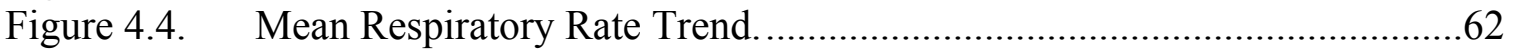

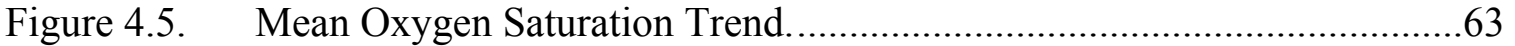

Figure 4.6. Mean Cardiac Output Trend. ...................................................................65

Figure 4.7. Mean Cardiac Index Trend. ..................................................................66

Figure 4.8. Pulmonary Artery Diastolic Pressure Trend.........................................67

Figure 4.9. Mean Pulmonary Artery Systolic Pressure Trend. ...................................69

Figure 4.10. Mean Mixed Venous Oxygen Trend...................................................... 70

Figure 4.11. Cardiopulmonary Indicators' Significant Differences over Time. ............80

Figure 5.1. Conceptual Model Indicating Significant Predictors of DVWR. ...........125 


\section{CHAPTER 1: INTRODUCTION}

Coronary artery bypass graft $(\mathrm{CABG})$ surgery is one of the most common classes of surgical procedures performed worldwide. Branca et al. (2001) reported that in the United States, more than 500,000 CABG procedures are performed every year (1). Recovery and stabilization of cardiopulmonary function is the major task of postoperative intensive care following surgery. The most important component of the postoperative intensive care services after CABG surgery includes weaning from mechanical ventilation and hemodynamic stabilization. Advances in fast track weaning protocols have decreased the required duration of postoperative mechanical ventilation after $\mathrm{CABG}$ surgery from a few days to a few hours (2-5). Findings from recent studies reveal that the time limits of normal ventilator weaning response (NVWR) after CABG surgery vary from one to ten hours $(1,6-25)$. Most patients are extubated within 6 to 8 hours after surgery. However, $20 \%$ to $40 \%$ of patients remain intubated 12 hours after surgery due to dysfunctional ventilator weaning response $(1,6-25)$.

Dysfunctional ventilator weaning response is a major problem among postoperative $\mathrm{CABG}$ patients because it is associated with increased morbidity and mortality following CABG surgery $(2,15,22,26-28)$. The mortality related to DVWR is reported as $30 \%$ to $43 \%(20,21)$. It results from many complications of DVWR such as acute respiratory distress syndrome, $10 \%$ to $15 \%(20,21)$; multiorgan dysfunction syndrome, $15 \%$ to $19 \%(20,21)$; deep vein thrombosis, $10 \%$ to $12 \%(29-31)$; and ventilator-associated pneumonia, $30 \%$ to $50 \%(32,33)$. Other health care problems resulting from DVWR are increased ICU length of stay and cost $(8,15,34,35)$. Early 
detection of DVWR is imperative to prevent postoperative complications and premature weaning after CABG surgery. Finding reliable predictive factors could help in the early detection of DVWR and might guide in planning early treatment for DVWR, and may aid in preventing complications and premature weaning (drop this red) after CABG surgery.

\section{Significance of the Study}

Prediction of DVWR is important because it may help to guide early identification of persons at risk for and in need of treatment to prevent DVWR. Prediction of DVWR also can help to prevent complications related to premature weaning trials after surgery on vulnerable patient populations. A review of literature reveals that there is a need for studies focusing on finding the predictive factors for DVWR. Many researchers have cited the need for studies to improve the criteria to predict early extubation and DVWR (36-40). Some researchers have examined the associated risk factors, readiness, feasibility, and effectiveness of early extubation (1, 625). Other researchers have focused on identifying the associated factors and complications of early extubation failure $(2,40-45)$. Although many researchers have attempted to identify the predictive factors for early extubation failure and prolonged mechanical ventilation (PMV), which is the outcome of DVWR, no single proven predictor is reported to be valid to predict DVWR $(34,37,46,47)$.

Although DVWR among postoperative CABG surgery patients is a critical problem associated with many postoperative complications such as PMV, increased ICU mortality, increased ICU length of stay, and increased ICU cost, very little has been done to predict the DVWR in this patient population. Previous researchers have used 
traditional respiratory parameters such as tidal volume, respiratory rate, and spontaneous minute ventilation to predict the weaning outcomes $(17,21)$. The findings of many research studies reveal that traditional respiratory parameters have poor predictive value in the weaning outcomes $(17,21)$. Some researchers have reported that hemodynamic variables can be used to predict the weaning outcomes (48-50). However, the prediction of DVWR using CPI is unexplored.

In past decades, research studies have focused on examining various weaning protocols and found that a fast track weaning protocol can be effectively and safely implemented among postoperative CABG patients $(8,15,34,35)$. Despite implementing fast track weaning protocols, there is a gap in the practice of weaning postoperative cardiac surgery patients, which is evidenced by the findings from many studies $(26,51)$. Some researchers have reported that $40 \%$ of ICU time is devoted in the weaning process, and $50 \%$ of self-extubated patients do not require reintubation $(12,52)$. Many research findings reveal that traditional respiratory parameters such as tidal volume, respiratory rate, and spontaneous minute ventilation have poor predictive value for DVWR and PMV.

In the weaning process, hemodynamic stability is a required criterion. Although the hemodynamic stability is the prerequisite for the initiation of the weaning process, little is known about its association with DVWR. Cardiopulmonary indicators (CPI) are the clinical hemodynamic variables monitored through invasive and non-invasive monitoring techniques in the ICU. The selected CPI to examine the predictive value in this study were heart rate (HR), mean arterial pressure (MAP), central venous pressure (CVP), cardiac output (CO), respiratory rate (RR), mixed venous oxygen saturation $\left(\mathrm{SVO}_{2}\right)$, oxygen saturation $\left(\mathrm{SPO}_{2}\right)$, pulmonary artery diastolic pressure $(\mathrm{PAD})$, and 
pulmonary artery systolic pressure (PASP). Changes in CPI precede the dysfunctional ventilator weaning response. They can be readily identified through hemodynamic trends by the bedside clinician. In addition, CPI is a sensitive and reliable clinical variable, which may be helpful to foresee the DVWR. Therefore, this research study was conducted to determine the values of CPI in predicting DVWR among postoperative CABG patients.

\section{Purposes of the Study}

The purposes of this research study were the following:

1. Describe the characteristics of CPI among patients with normal ventilator weaning response (NVWR) and dysfunctional ventilator weaning response (DVWR) after CABG surgery.

2. Find the differences in characteristics of cardiopulmonary indicators between patients with normal ventilator weaning response (NVWR) and dysfunctional ventilator weaning response (DVWR) after CABG surgery.

3. Build a prediction model for dysfunctional ventilator weaning response (DVWR) with significant antecedence.

\section{Research Questions of the Study}

This research study was guided by the following research questions in patients after coronary artery bypass graft surgery: 
1. What are the characteristics of cardiopulmonary indicators in patients with dysfunctional ventilator weaning response (DVWR) and normal ventilator weaning response (NVWR)?

2. What are the differences in the characteristics of cardiopulmonary indicators in patients with dysfunctional ventilator weaning response (DVWR) and normal ventilator weaning response (NVWR)?

3. What is the best predictive model for dysfunctional ventilator weaning response (DVWR)?

\section{Assumptions}

The following assumptions were made:

1. CPI are measured following a standard protocol, and they are accurate and reliable.

2. All patients in the study population underwent a standard weaning protocol.

3. The patients' records are stored and maintained in a standard system and are, therefore, accessible for data retrieval.

4. Clinical factors that are associated with DVWR are associated with changes in selected CPI.

\section{Definitions}

A key concept in this study was that of cardiopulmonary indicators (CPI). These indicators are defined as the selected hemodynamic variables recorded postoperatively in the cardiovascular intensive care unit (CVICU) after CABG surgery. Within this study CPI included $\mathrm{HR}, \mathrm{MAP}, \mathrm{CVP}, \mathrm{CO}, \mathrm{RR}, \mathrm{SVO}_{2}, \mathrm{SPO}_{2}, \mathrm{PAD}$, and PASP, which were 
retrieved from the electronic patient file (EPF) of postoperative CABG patients. An electronic patient file is an electronic medical record in which the individual patient's information, clinical data, treatments, and outcome are recorded and maintained by the study hospital. A definition for each CPI indicator follows:

1. Heart rate $(H R)$. The actual numeric value of heartbeat recorded by the Spacelab cardiac monitor and electronically transferred to the patient's vital sign computer charting. It is retrieved from the nursing assessment page of the EPF.

2. Mean arterial blood pressure (MAP). The actual numeric value of the MAP recorded by Spacelab cardiac monitor through the arterial line and electronically transferred to the patient's vital sign computer charting. It is retrieved from the nursing assessment page of the EPF.

3. Central venous pressure (CVP). The actual numeric value of CVP recorded through the pulmonary artery catheter by the Spacelab cardiac monitor and electronically transferred to the patient's vital sign computer charting. It is retrieved from the nursing assessment page of the EPF.

4. Cardiac output (CO). The actual numeric value of cardiac output recorded through the pulmonary catheter by the Edwards Lifesciences $\mathrm{SVO}_{2}$ monitor and entered into the nursing assessment page; it is retrieved from the nursing assessment page of the EPF.

5. Respiratory rate (RR). The actual numeric value of the patient's total ventilator rate per minute recorded by the ventilator and entered into the nursing assessment page; it is retrieved from the nursing assessment page of the EPF. 
6. Mixed venous oxygen saturation $\left(\mathrm{SVO}_{2}\right)$. The actual numeric value of $\mathrm{SVO}_{2}$ recorded through the pulmonary artery catheter by the Edwards Lifesciences $\mathrm{SVO}_{2}$ monitor and entered in the nursing assessment page; it is retrieved from the nursing assessment page of the EPF.

7. Oxygen saturation $\left(\mathrm{SVO}_{2}\right)$. The actual numeric value of the $\mathrm{SVO}_{2}$ recorded by the Spacelab cardiac monitor through a Nelcore pulse oxymetry probe and electronically transferred to the patient's vital sign computer charting. It is retrieved from the nursing assessment page of the EPF.

8. Pulmonary artery diastolic pressure $(P A D)$. The actual numeric value of PAD recorded through the pulmonary artery catheter by the Spacelab cardiac monitor and electronically transferred to the patient's vital sign computer charting. It is retrieved from the nursing assessment page of the EPF.

9. Pulmonary artery systolic pressure (PASP). The actual numeric value of PASP recorded through the pulmonary artery catheter by the Spacelab cardiac monitor and electronically transferred to the patient's vital sign computer charting. It is retrieved from the nursing assessment page of the EPF.

10. Dysfunctional ventilator weaning response (DVWR). DVWR is operationally defined as the condition in which the patient remains on the ventilator $>8$ hours after surgery. It is identified as the ventilator setting charted after 8 hours of surgery in the nursing assessment and nursing notes pages of the EPF.

11. Normal ventilator weaning response (NVWR). Normal ventilator weaning response is operationally defined as the condition in which the patient is extubated and placed on either a binasal cannula or an aerosol facemask before 8 hours after surgery. It is the 
first charted time of BiNasal cannula (BNC) or aerosol facemask (AFM) oxygen after surgery that is recorded in the nursing assessment and nursing notes pages of the EPF.

\section{Limitations}

Although utilizing a retrospective study design was suitable and appropriate for the research problem, this design has both strengths and limitations. The readily available data is a strength for the study, as it prevents a data collection bias and helps to reveal existing relationships as they are dictated by the available data. However, this design does not exhibit the examination of cause and effect relationships between the study variables.

Another limitation of this research study was that the researcher had no control over the completeness of the available data. Completeness and accuracy of the available data are two critical elements to consider in this design. Data extraction is another step where there is possibility of data error. A systemic chart extraction format and counter verification with another peer in the discipline controlled the accuracy of the data extraction.

The generalizability of the study's findings is limited because it is a single site study. This study finding is limited to adult postoperative CABG patients and is not applicable to pediatric population or any other postoperative adult patients. 


\section{CHAPTER 2: REVIEW OF LITERATURE}

This review of literature involved analysis of past research literature utilizing Medline (CSA) and PubMed (NLM) search engines via the Endnote program. Literature related to the research problem, involving various disciplines and perspectives of the research problem, was included in this research study. It is organized under headings such as Definition of DVWR, Defining Characteristics of DVWR, Prevalence and Risk Factors, Prediction in Prevention of DVWR, Cardiopulmonary Indicators in Prediction of DVWR, and Conceptual Framework. The literature in the conceptual framework included these topics: DVWR, CPI, perioperative risk factors of CABG surgery, ventilator weaning after CABG surgery, cardiopulmonary monitoring after CABG surgery, and mechanical ventilation after CABG surgery.

\section{Definition of Dysfunctional Ventilator Weaning Response}

Dysfunctional ventilator weaning response (DVWR) is defined as an "inability to adjust to lowered levels of mechanical ventilator support that interrupts and prolongs the weaning process" $(17,20,21,55,56)$. Dysfunctional ventilator weaning response is a major problem among postoperative $\mathrm{CABG}$ patients. It is associated with a lethal complication known as prolonged mechanical ventilation (PMV). Prolonged mechanical ventilation is defined as a condition in which the postoperative $\mathrm{CABG}$ patient is unable to be extubated at 24 hours after surgery and needs mechanical ventilation support more than 24 hours $(55,56)$. 


\section{Defining Characteristics of Dysfunctional Ventilator Weaning Response}

The defining characteristics of DVWR depend upon the severity of the condition. It can be mild, moderate, or severe. The majority of the defining characteristics include alteration in hemodynamic clinical data, which can be measured objectively. The signs and symptoms of mild DVWR include slight increased respiratory rate (RR) from the baseline and excessive use of respiratory muscles. The signs and symptoms of moderate DVWR include increase in blood pressure, increase in heart rate, increase in respiratory rate, mild cyanosis, decrease in oxygen saturation from the baseline, and excessive use of accessory muscles for respiration. The signs and symptoms of severe DVWR include deterioration in arterial blood gasses, increase in blood pressure, increase in heart rate, increase in respiratory rate, cyanosis, gasping, altered mental status, and decrease in oxygen saturation (55-58).

\section{Prevalence and Risk Factors}

Yende and Wunderlink (2002) reported that $20 \%$ to $40 \%$ of patients continue to receive PMV after undergoing CABG surgery due to DVWR (21). The causes of PMV after CABG surgery are heterogeneous, vary with time, and have a variable impact on the duration of mechanical ventilation required after the patient undergoes CABG surgery. Hypoxemia was found to be the most common cause for PMV for $>24$ hours $(5,59-62)$. Several researchers reported the causes for prolonged ventilation after heart surgery as pulmonary edema $(25 \%)$, ARDS (5\%), excessive bleeding (10\%), and tachypnea during weaning trials $(16.2 \%)(20,21,63,64)$. Some research findings reveal that increased age $>70$ years is associated with PMV $(8,19,65-67)$. In contrast, recent research findings 
reveal that the outcome of CABG surgery has progressively improved despite increased numbers of elderly patients and worsened preoperative risk profiles over the past few decades, and increased age is not associated with $\operatorname{PMV}(1,2,7,12)$.

There are many clinical variables associated with DVWR. Clinical variables such as chronic obstructive pulmonary disease (COPD), congestive heart failure (CHF), emergency surgery, and number of grafts are found to be associated with DVWR and PMV $(6,7,12,20,21,68)$. Some researchers reported that DVWR is associated with the adult respiratory distress syndrome after CABG surgery $(6,16,24,58,69-71)$. Another common complication associated with DVWR following cardiac surgery is deep vein thrombosis. Findings from some studies reveal that $23.6 \%$ of patients who required prolonged mechanical ventilation developed deep vein thrombosis despite $100 \%$ prophylaxis. Multiorgan dysfunction syndrome is also a complication associated with DVWR and PMV (29-31). The prevalence of multiorgan dysfunction syndrome among patients with PMV after cardiac surgery is estimated as $22.7 \%$ (29-31). Ventilatorassociated pneumonia is the most frequent complication associated with DVWR and PMV. Approximately $50 \%$ of ventilated patients develop ventilator-associated pneumonia within the first 4 days of mechanical ventilation (29-31).

Furthermore, DVWR is a critical problem, as it is associated with intensive care unit (ICU) mortality following CABG surgery $(2,15,22,26-28)$. The mortality related to DVWR is reported as $43 \%(20,21)$. The mortality after heart surgery is associated with many complications of DVWR such as acute respiratory distress syndrome, multiorgan dysfunction syndrome, deep vein thrombosis, and ventilator-associated pneumonia. The ICU mortality related to acute respiratory distress syndrome is reported as $15 \%(32,33)$. 
The ICU mortality attributable to multiorgan dysfunction syndrome is reported as $19.6 \%$ (29-31). Another common cause of ICU mortality is ventilator-associated pneumonia. The Institute of Hospital Improvement (2004) Report revealed that ventilator-associated pneumonia is a leading cause of morbidity and mortality in the ICU resulting from DVWR and PMV. The ICU mortality associated with ventilator-associated pneumonia is $30 \%$ to $50 \%(20,21)$.

Other health care problems resulting from DVWR are increased ICU length of stay and ICU cost $(8,15,34,35)$. Providing critical care consumes $30 \%$ of the hospital expenses for just $8 \%$ of the hospital population (14). Several researchers who focused on studying ICU length of stay and early extubation after cardiac surgery reported that increased ICU length of stay and ICU cost is directly associated with DVWR and PMV $(8,15,34,35)$. Prolonged mechanical ventilation $>48$ hours accounted for $42.8 \%$ of the total ICU cost (14).

As a solution to the problem, recent research findings reveal that hemodynamic variables have predictive values in ventilator weaning $(14,72)$. Some researchers have reported that CPI is associated with ventilator weaning outcomes, particularly with PMV (58). However, no reported study focused on selected CPI, which may be used as predictive indicators for DVWR.

\section{Prediction in Prevention of Dysfunctional Ventilator Weaning Response}

Lack of prediction of DVWR may lead to premature weaning trials among highrisk population. Premature weaning trials and difficulty in weaning may result in risks of myocardial ischemia during the postoperative period because of increased global oxygen 
consumption associated with recovery and ventilator weaning $(58,73,74)$. Weaning involves increased oxygen demand and increased cardiopulmonary stress. Some studies have demonstrated the increased amount of oxygen consumption $\left(\mathrm{VO}_{2}\right)$ and stress level during weaning. De Backer, et al. (2000) reported that there is 5\% increase in the cardiac index $(\mathrm{CI})$ and a $10 \%$ increase in $\mathrm{VO}_{2}$ during the weaning of patients from the mechanical ventilator after heart surgery (75). Alteration in the cardiac index during weaning is found to have an association with weaning failure $(15,76-78)$. Meade, et al. (2001) reported that failed trials and premature trials of discontinuation of mechanical ventilation may precipitate respiratory muscle injury and result in prolonged mechanical ventilation (37). Thus, it is highly important to predict the DVWR to help in preventing premature trials and complications.

Given the fact that there is a risk of silent myocardial ischemia during the weaning process, critical care clinicians must carefully weigh the benefits of early weaning from mechanical ventilation against the risk of premature trials of early extubation. The need for accurate prediction is mandatory in order to prevent DVWR. If predictive values of CPI could be determined, clinicians could prevent premature trials and DVWR. Postoperative cardiopulmonary dysfunction is most common complication among CABG patients. However, the postoperative cardiopulmonary course events from surgery to DVWR are unknown and unexplored. Although there is much literature available on preoperative risk factors, little is known about postoperative cardiopulmonary events predisposing to DVWR (75). Determination of the predictive values of CPI in DVWR will promote patient safety and satisfaction and reduce ICU mortality, length of hospital stay, and health care cost. 


\section{Cardiopulmonary Indicators in Prediction of Dysfunctional Ventilator Weaning Response}

Clinically, hemodynamic stabilization is monitored through CPI. The selected CPI to predict DVWR in this research study include heart rate (HR), mean arterial blood pressure (MAP), central venous pressure (CVP), cardiac output (CO), respiratory rate (RR), mixed venous oxygen $\left(\mathrm{SVO}_{2}\right)$, oxygen saturation (54), pulmonary artery diastolic pressure (PAD), and pulmonary artery systolic pressure (PASP).

In the weaning process, hemodynamic stability is a required criterion. Although hemodynamic stability is the prerequisite for initiating the weaning process, there is little known about its association with DVWR. Changes in CPI can be readily identified through hemodynamic trends. Cardiopulmonary indicators are sensitive clinical variables, which may be helpful to foresee DVWR. Therefore, this research study is conducted to determine the predictive values of CPI with regard to DVWR among postoperative CABG patients.

There is reported evidence of an association between some CPI and weaning outcomes such as NVWR and DVWR (30, 79-83). Frazier, et al. (2001) reported that hemodynamic instability during weaning from mechanical ventilation is one proposed cause of weaning failure (84). Jubran, et al. (1998) reported a direct association between $\mathrm{SPO}_{2}$ level above $92 \%$ and NVWR (85). Findings from some research reveal that the increase in MPASP and the change in CO during the weaning process are associated with weaning failure (86). A progressive decrease in $\mathrm{SVO}_{2}$ during the weaning process is

reportedly associated with weaning failure $(87,88)$. Increased respiratory rate during the 
weaning process is also associated with weaning failure (20,33). Doering, et al. (2001) reported that early hemodynamic instability and presence of arrhythmias are associated with weaning failure and postoperative mortality (76). Estenssoro, et al. (2005) reported that clinical conditions such as low cardiac output, increased heart rate, apprehension, deterioration of blood gas values, and slight respiratory accessory muscle use had the highest association with DVWR (89). Ely, et al. (1996) reported that increases in RR, HR, and MAP are associated with DVWR $(36,90)$. Alteration in cardiac index during weaning has been found to have association with weaning failure $(15,76-78)$. Although many researchers have reported the association between CPI and weaning outcomes, there is no reported research using selected CPI for the prediction of DVWR.

Using CPI to predict DVWR has many advantages, such as being sensitive indicators, reliable in detecting the complications beyond the associated comorbidity, helpful in early diagnosis, and feasible to implement in practice by the nurses in detecting DVWR and preventing premature trials. Cardiopulmonary indicators are sensitive indicators in identifying complications among cardiac surgery patients (91-94). Some researchers have reported that the trends of hemodynamic variables for 4 hours following ICU admission after CABG surgery are sensitive indicators of prognosis (95).

In addition, CPI are reliable in detecting complications after heart surgery irrespective of comorbid factors associated with outcomes (96). Furthermore, some researchers have reported that cardiopulmonary indicators are helpful in rapid diagnosis of postoperative complications among heart surgery patients $(97,98)$. Other research findings reveal that changes in CPI are reliable indicators of postoperative adverse events (92). 
Weaning is an important aspect of critical care nursing for postoperative CABG surgery patients. Determination of optimum cardiopulmonary function is a prerequisite for the weaning process. Restoration and maintenance of normal cardiopulmonary function without injuring the heart and other organs represent the most important goal in the nursing care of postoperative CABG surgery patients (26). Continuous postoperative CPI monitoring to prevent postoperative complications in the ICU is a known practice. The critical care nurse plays an important role in the prevention of postoperative complications through CPI monitoring. The primary functions of the critical care nurse during the postoperative period are CPI monitoring, hemodynamic stabilization, and assessing weaning readiness of the patient. Thus, the critical care nurse plays an important role in predicting DVWR through CPI.

Although the fast track weaning protocols driving early extubation have economic implications, it is imperative to consider that there are a considerable number of postoperative CABG patients at risk of developing DVWR. Weaning from mechanical ventilation involves a significant amount of stress to physiological systems and increased risk of postoperative silent myocardial ischemia. Calzia, et al. (2001) reported evidence of increased plasma levels of epinephrine, nor epinephrine, cortisol, vasopressin, and prolactin during the weaning process, all of which are the indicators of stress (68). Some researchers reported that the weaning process has a high risk for silent myocardial ischemia among the cardiac risk population during the non-cardiac surgery postoperative period (99). 


\section{Conceptual Framework}

This research study examined the predictive value of CPI for DVWR among postoperative $\mathrm{CABG}$ surgery patients. The three main concepts involved in this study were CPI, DVWR, and NVWR. The associated concepts to DVWR and CPI included in this study were perioperative risk factors of CABG surgery, ventilator weaning, cardiopulmonary monitoring, and mechanical ventilation.

Cardiopulmonary indicators: Cardiopulmonary indicators (CPI) are the clinical hemodynamic variables monitored through invasive and non-invasive monitoring techniques in the ICU. The selected CPI to examine the predictive value of in this study are $\mathrm{HR}, \mathrm{MAP}, \mathrm{CVP}, \mathrm{CO}, \mathrm{RR}, \mathrm{SVO}_{2}, \mathrm{SPO}_{2}, \mathrm{PAD}$, and PASP. The postoperative CABG patient's CPI is measured through invasive hemodynamic monitoring by pulmonary artery (PA) catheters, bedside cardiac monitor, $\mathrm{SVO}_{2}$ monitor, pulse oxymetry, pressure transducers, arterial line, and ventilator. The definition, physiology, and significance of individual CPI in prediction of DVWR are explained in the following paragraphs.

Heart rate: Heart rate (HR) is the number of mechanical cardiac cycles per minute. Cardiac cycle refers to one complete mechanical action of the heartbeat (58). Heart rate is monitored continuously through the cardiac monitor in the postoperative cardiovascular ICU. The normal heart rate is 60 to 100 per minute. Physiologically, changes in heart rate are accomplished by reflex controls mediated by the autonomic nervous system, including its sympathetic and parasympathetic divisions. The parasympathetic impulses, which travel to the heart through the vagus nerve, can slow the 
heart rate, whereas sympathetic impulses increase the heart rate. These effects on heart rate result from action on the sino atrial node to either increase or decrease its rate. The balance between these reflex control systems determines the heart rate. Heart rate may also be increased in the presence of excessive thyroid hormones, catecholamines, and increased metabolic demands. In addition, an increase in heart rate may be seen as the compensatory mechanism of increased circulatory and oxygen demand. Findings from an animal study on the effects of hypoxia on heart rate revealed that hypoxia increased heart rate significantly as an earliest compensatory mechanism (100). Heart rate may be used as an early sensitive indicator of cardiopulmonary problems. Heart rate is considered a sensitive parameter by many researchers to assess the cardiac and circulatory status of the postoperative cardiac patients (101). Changes in heart rate are an indication of cardiac compensation during various stress and activities.

The significance of including HR to predict DVWR emerges from the physiological changes in HR during the weaning process. Weaning from ventilation after CABG surgery involves stress to the heart, which is reflected as increased heart rate during the weaning process. A prospective comparative study between patients with betablocker and without beta-blocker revealed a significant increase in heart rate in both groups during weaning. The increase in heart rate among patients with beta-blocker was $20 \%$ above baseline and in patients without beta-blocker was $>20 \%$. The results of another comparative study on hemodynamic changes during the weaning process among patients with and without heart diseases revealed a significant increase in heart rate and rhythm in patients with heart disease (102). 
Mean arterial blood pressure: Mean arterial blood pressure (MAP) is the average pressure exerted on the walls of the arteries (58). It is affected by various factors such as cardiac output, distension of the arteries, and the volume, velocity, and thickness of the blood. Blood pressure occurs as a cyclic phenomenon. The pressure during contraction of the ventricle is called systolic pressure, and the pressure during relaxation of the ventricle is called diastolic pressure.

The normal physiology of blood pressure regulation is through a complex interaction of neural, chemical, and hormonal feedback systems affecting both cardiac output and peripheral vascular resistance. Tissue and organ perfusion depend on mean arterial pressure (MAP). The MAP is the average pressure at which blood moves through the vasculature. The normal range of MAP is 80 to $120 \mathrm{mmHg}$.

The significance of including MAP in predicting DVWR is based on the evidence of increased MAP with many postoperative complications. Some researchers have reported that increased mean arterial pressure is associated with postoperative complications after CABG surgery $(101,103)$. Researchers have also reported that measurement of mean arterial pressure is useful in predicting postoperative complications $(101,104)$. A comparative study on the effect of weaning in hemodynamic changes revealed a significant increase in MAP in patients with beta-blocker and in patients without beta-blocker during the weaning process. There was a $40 \%$ increase in MAP above baseline among those patients who had no beta-blocker and a $23 \%$ increase among patients with beta-blocker (105). 
Central venous pressure: Central venous pressure (CVP) is a dynamic or changing measurement. The change in CVP, correlated with a patient's clinical status, is a more useful indication of the adequacy of venous blood volume and alterations of cardiovascular function than a single measurement. Central venous pressure reflects the right ventricular function. Most right ventricular failure is secondary to left ventricular failure. Therefore, an elevated CVP can be either a late sign of left ventricular failure or increased fluid volume. A decreased CVP indicates that the patient is in hypovolemia. The normal CVP is 4 to $10 \mathrm{~cm} / \mathrm{H}_{2} \mathrm{O}(58)$.

Measurement of CVP is a guide to assess the preload and fluid status postoperatively. The significance of adding CVP to predict DVWR is based on the evidence that increased positive fluid balance is associated with PMV (106). Postoperative $\mathrm{CABG}$ patients with mechanical ventilation are at risk of developing altered preload and fluid status. Mechanical ventilation is associated with alterations in intrathoracic pressure, lung volume, and venous return; and changes in intrathoracic fluid volumes during weaning are associated with weaning failure. In patients with impaired cardiac function, the development of pulmonary edema during weaning has been described (58). An investigation of changes in intrathoracic fluid volumes in patients after coronary artery bypass grafting after changing the ventilatory pattern from mechanical to spontaneous ventilation revealed that increased preload during the weaning process is associated with weaning failure (107).

Cardiac output: Cardiac output (CO) is defined as the volume of blood ejected from the heart over 1 minute (58). The determinants of $\mathrm{CO}$ are heart rate and stroke 
volume. Changes in either stroke volume or heart rate change the $\mathrm{CO}$. The continuous measurement of $\mathrm{CO}$ permits the clinician to assess the trends in the cardiovascular status of the patient status at the bedside. Trends in the patient's cardiovascular status can be observed by using serial CO measurements. Normal CO is 4 to $81 / \mathrm{min}$ (58). Some studies have found that low cardiac output is associated with DVWR $(20,37)$. Some researchers either reported that an acute increase in oxygen demand can be compensated for by increased cardiac output $(\mathrm{CO})$ or increased oxygen extraction, resulting in reduced mixed venous oxygen saturation $\left(\mathrm{SVO}_{2}\right)$. A postoperative increase in $\mathrm{CO}$ and heart rate is found to be an indication of an increase oxygen demand (88). Assessing the trend of CO measurements may help to predict the DVWR.

Mixed venous oxygen saturation: Although $\mathrm{CO}$ provides important information about the capacity of the cardiopulmonary system to deliver oxygen to the tissue, it does not depict the adequacy of the oxygen supply at the tissue level. The assessment of tissue level metabolism is determined by mixed venous oxygen saturation $\left(\mathrm{SVO}_{2}\right)$. The $\mathrm{SVO}_{2}$ measurement reflects the $\mathrm{O}_{2}$ utilization by all the tissues in the body (58). Continuous monitoring of $\mathrm{SVO}_{2}$ is done through a PA catheter. The $\mathrm{SVO}_{2}$ measurement provides an index of tissue metabolism and can be used as an adjunct to therapy when making adjustments in ventilator settings and weaning. In addition, the $\mathrm{SVO}_{2}$ provides immediate feedback of the patient's cardiopulmonary status, and it is effective in evaluating the therapy and patient status $(32,33)$. Although the usefulness of the $\mathrm{SVO}_{2}$ is recognized clinically, the association of $\mathrm{SVO}_{2}$ with DVWR is unknown. Some studies have found an association between the decrease in $\mathrm{SVO}_{2}$ after extubation and reintubation $(37,73,108)$. 
Findings from an experimental clinical study reveal that measures of $\mathrm{SVO}_{2}$ are sensitive to changes in oxygenation status during weaning. The $\mathrm{SVO}_{2}$ measures provide valid information about oxygen status and can be used to guide weaning (86).

Respiratory rate and oxygen saturation: Respiration is defined as the process of inspiration and expiration which facilitates ventilation in the lungs (58). The rhythmicity of respiration is controlled by the respiratory centers in the brain. The process of inspiration and expiration is controlled by medulla oblongata, and the rate and depth of the respiration is controlled by pons. The normal respiratory rate (RR) is 12 to 18 breaths per minute. Observing respiratory rate is important because changes in the respiratory rate are an indicator of an altered cardiopulmonary status. Increased RR is reported to be associated with cardiopulmonary stresses and compensatory effort to combat hypoxia (36, $60,109)$. The measurement of $\mathrm{RR}$ and oxygen saturation $\left(\mathrm{SPO}_{2}\right)$ is also useful to predict pulmonary status. Some researchers found that $\mathrm{RR}$ and $\mathrm{SPO}_{2}$ are valuable predictors of arterial blood gases. Monitoring RR and $\mathrm{SPO}_{2}$ postoperatively may assist in patient monitoring during weaning and reduce the number of arterial blood gases needed $(59,86)$. Research findings on weaning elderly patients reveal that RR is a sensitive indicator to predict the weaning outcome (110).

\section{Pulmonary artery diastolic pressure and pulmonary artery systolic pressure:}

Pulmonary artery pressures, both diastolic (PAD) and systolic (PASP), provide an index of the pressure within the pulmonary vasculature and are affected by the left ventricular compliance. These pressures depend on blood flow to the lungs and the state of the lung 
tissue. In the absence of pulmonary disease, the pulmonary artery pressure is equal to right ventricular pressure during systole. The normal value of pulmonary artery pressures (111) are 15 to $30 \mathrm{mmHg}$ (systolic) and 4 to $12 \mathrm{mmHg}$ (diastolic) (58).

The significance of including PAD and PASP as predictors of DVWR is from the theoretical evidence of the association between pulmonary hypertension and weaning failure after heart surgery (112-116). Changes in pulmonary artery pressure are sensitive indicators of altered cardiopulmonary and fluid status within the circulatory system. Elevated pulmonary artery pressures are an indication of pulmonary hypertension. Pulmonary hypertension is directly associated with DVWR (58). Continuous monitoring of pulmonary artery pressures may help to identify and treat pulmonary hypertension. Elevated pulmonary artery pressures are seen in COPD, left ventricular failure, pulmonary hypertension, hemothorax, pneumothorax, and DVWR $(32,33)$.

Mechanical ventilation imposes changes in intrathoracic pressure that are opposite to what occurs during normal respiration, which has an effect on the pulmonary artery pressures (58). During the inspiratory cycle of ventilation, the PAP increases, and during the expiratory cycle of ventilation, PAP decreases. Some studies reported that an increase in PEEP increases PAP $(30,111,117-119)$. On the other hand, some studies demonstrated that there is no difference in the PAP readings with PEEP of $10 \mathrm{~cm}$ or less $(1,2,5)$. Pulmonary hypertension with associated right ventricular dysfunction may complicate the postoperative CABG surgery patient despite maximum pharmacologic and ventilatory support. Continuous monitoring of pulmonary artery pressures may help to identify and treat pulmonary hypertension (120). 


\section{Effects of comorbidity in alteration of cardiopulmonary indicators:}

Comorbidities such as COPD, CHF, and renal disease are associated with alterations of the CPI. Many researchers have reported the alterations in CPI among COPD patients. The predominant risk factors that have an association with altered cardiopulmonary function are preoperative comorbid conditions such as chronic obstructive pulmonary disease (COPD), congestive heart failure (CHF), and renal disease.

A common cause for the alteration in CPI due to COPD is autonomic neuropathy. Autonomic neuropathy is a common complication associated with COPD. Autonomic neuropathy results in hemodynamic alterations in patients with COPD; it alters the baseline hemodynamic parameters among these patients such as pulmonary artery pressures, respiratory rate, and oxygen saturation (121). Another complication of COPD is breathing pattern alterations resulting in alterations of CPI. Breathing pattern alteration in a COPD patient is a common finding; it alters hemodynamic status. Particularly in mechanically ventilated patients with COPD, the pattern of lung inflation and expiratory time alteration have a significant impact on respiratory system mechanics, gas exchange, and hemodynamics (122). Another phenomenon involving alteration in CPI among COPD patients is salt and water accumulation due to the vasodilator properties of hypercapnia. The consequent low arterial blood pressure may be the stimulus for the neurohormonal activation and retention of salt and water (123). Findings from a clinical study revealed that significant increase in renal plasma flow, increased CVP, pulmonary artery pressures, and decreased cardiac output is associated with COPD. Another researcher reported that patients with COPD markedly increase their pulmonary artery wedge pressure on mild exercise even though they have no overt left heart disease (124). 
The effect of CHF on CPI is due to the cardiopulmonary dynamics. Left ventricular failure is associated with increased pulmonary pressures and decreased cardiac output. In addition, cardiac surgery depresses the myocardial pump function (80). Right heart failure is associated with increased right atrial pressure and impeding venous return and venous congestion alters hemodynamics $(125,126)$. A compensatory mechanism of CHF also includes increased sympathetic nervous system activity due to increased angiotensin converting enzymes which results in increased heart rate (127).

The effect of renal diseases in altering CPI is reported to be associated with the adoptive mechanism. The adoptive mechanism in renal disease includes volume overload and pressure overload that are responsible for adoptive alterations of the heart and vessels. The persistence of the adaptive mechanism leads to a detrimental process, mainly cardiac dilation, and failure and alteration in hemodynamics (128). Renal disease patients exhibit various hemodynamic alterations such as hypertension, left ventricular hypertrophy, and altered cardiac output (129). Renal failure also predisposes to alterations in left ventricle structure, aortic structure, and performance. Many pathogenesis related to left ventricular hypertrophy, such as high cardiac output, are related to renal anemia, hypertension, volume overload, and an arteriovenous fistula (130). Renal anemia predisposes to alterations in CPI because it induces functional and organic alterations of cardiaccirculatory function. One clinical researcher reported that patients with severe anemia showed a tendency to an impaired cardiac index below $\mathrm{Hb}<5-6 \mathrm{~g} / \mathrm{dl}$ with no alterations in pulmonary artery pressure (131). Changes in the structure of the aorta include endovascular deposition of microparticles from platelet aggregation resulting in stiffness and stenosis, which also predispose to concentric left ventricular hypertrophy, which is 
associated with diastolic dysfunction and alterations in CPI (132). Hemodynamic alterations among renal disease patients from chronic renal ischemia are a common pathophysiology. Another clinical study revealed evidence of chronic renal arterial ischemia producing many of the hemodynamic alterations and end-organ injury seen in patients with renal artery stenosis, including persistent hypertension, renal insufficiency, and cardiac disturbance syndromes (133). Another author proposed the uric acid hypotheses as an explanation for the alterations in CPI among renal disease patients. The researcher demonstrated hyperuricemia as a cause for the alteration in blood pressure and renal damage in an experimental animal study (134).

Cardiopulmonary monitoring: Cardiopulmonary monitoring is an associated concept with CPI in this research study. Cardiopulmonary monitoring is accomplished through hemodynamic monitoring that helps to prevent complications after heart surgery $(80,135)$. A complete assessment of all systems is performed when the patient is admitted to the postoperative cardiovascular intensive care unit, and every hour thereafter until 8 hours following the ICU admission to determine postoperative status of the patient. Cardiopulmonary assessment is performed to monitor the cardiac and respiratory status of the patient. Cardiac assessments performed in the postoperative cardiovascular ICU include heart rate, heart sounds, arterial blood pressure, central venous pressure, pulmonary artery pressure, pulmonary artery wedge pressure, left atrial pressure, cardiac output, cardiac index, systemic vascular resistance, pulmonary vascular resistance, mixed venous oxygen saturation, and pacemaker status. 
The pulmonary assessments performed in the postoperative cardiovascular ICU include chest movement, breath sounds, ventilator settings, oxygen saturation, respiratory rate, chest tube drainage, and arterial blood gases. Nursing management of postoperative CABG patients involves continuous monitoring of a patient's cardiopulmonary status and relaying changes to the surgeon and critical care team work to correct the problem collaboratively. In evaluating the cardiopulmonary status, the nurse performs clinical observations and routine measurements such as serial readings of blood pressure, heart rate, central venous pressure, arterial pressure, cardiac output, cardiac index, pulmonary artery pressure, oxygen saturation, respiratory rate, and mixed venous oxygen saturation.

The assessment of cardiopulmonary status is performed through hemodynamic monitoring, which involves the use of invasive catheters in the vascular system of patients. The specific monitoring catheters and transceducers used in the hemodynamic monitoring are the pulmonary artery catheter and systemic arterial catheter.

The pulmonary artery catheter is an important assessment tool that is useful to measure and calculate several right and left sided intracardiac pressures effectively. The pulmonary artery catheter is a balloon-tipped, flow-directed catheter inserted into the superior vena cava and right atrium. The balloon is inflated, and the catheter is carried rapidly by the flow of the blood through the tricuspid valve into the right ventricle, through the pulmonic valve, and into the branch of the pulmonary artery. With the pulmonary artery catheter, many parameters can be measured, including CVP, PASP, PADP, $\mathrm{CO}, \mathrm{CI}, \mathrm{SvO}_{2}$, and pulmonary artery capillary wedge pressure. Systemic arterial pressure monitoring is used to obtain direct and continuous blood pressures in the 
postoperative cardiovascular ICU. Arterial catheters are also useful in assessing frequent arterial blood gases.

Dysfunctional ventilator weaning response: Many nomenclatures are used to denote DVWR, such as dysfunctional weaning, early extubation failure, DVWR, and prolonged mechanical ventilation (PMV). Many studies have defined this term operationally as the condition in which the patient is unable to be weaned from the ventilator and remains intubated and on mechanical ventilation $>8$ hours after surgery ( 1 , 6-25). Based on the review of literature, this study operationally defines DVWR as the condition in which patients remain on the ventilator $>8$ hours after surgery. In addition, the term normal ventilator weaning response (NVWR) is operationally defined as the condition in which the patient is extubated and placed on either a binasal cannula or aerosol facemask oxygen within 8 hours after surgery.

\section{Perioperative risk factors of coronary artery bypass graft surgery associated} with dysfunctional ventilator weaning response: There are many perioperative risk factors associated with DVWR. Patients with coronary artery disease are particularly at risk perioperatively from myocardial infarction, unstable angina, severe arrhythmia, and cardiac death $(136,137)$. Perioperative risk factors that are associated with DVWR vary distinctively with the preoperative period, intraoperative period, and postoperative period. Pulmonary complications are identified as major risk factors for postoperative mortality. It is important to identify the cardiopulmonary variables that are critical to predict 
DVWR and related mortality in the postoperative period after coronary artery bypass grafting (138).

Many preoperative comorbid conditions, which exist in patients before surgery have been found to be associated with DVWR. The reported associated comorbid conditions include increased age, female gender, congestive heart failure, chronic obstructive pulmonary disease, redo surgery, renal dysfunction, and cerebrovascular disease (1, 6-25).

Many intraoperative factors have been found to influence the timing of postoperative extubation after CABG surgery. Intraoperative risk factors that are associated with DVWR include comorbid conditions, anesthesia, and surgical techniques. The reported intraoperative comorbid factors that are associated with DVWR include ejection fraction $<40 \%$, prolonged cardio pulmonary bypass time $>120$ minutes, reexploration, and emergency surgery. $(1,12,18,19,139-143)$.

Another major intraoperative factor, which is associated with postoperative ventilation time, is anesthesia. The type of anesthesia and its interaction with comorbid conditions such as age and poor ventricular functions has been associated with prolonged postoperative ventilation $(1,12,18,19,139-143)$. Fast track anesthesia has been found to have an effect in early extubation $(2,3,6,8,9,12,13,15,16,40,72,73,144-149)$.

Some authors have reported that surgical techniques such as number of grafts and use of internal mammary artery (150) graft influence the time of postoperative ventilation (151). Minimally invasive procedures, such as beating heart surgeries, have been found to have an effect in early extubation and reduced ICULOS (2). 
Many postoperative risk factors are associated with DVWR, such as increased duration of mechanical ventilation, lack of weaning protocols, premature weaning trials, hemodynamic instability, and altered fluid balances. Increased duration of mechanical ventilation is associated with acute lung injury complicating DVWR $(2,40-45,51,140$, 143, 152-154). A lack of standard weaning protocols is associated with $\operatorname{PMV~}(2,3,17,65$, 155-165). Another postoperative risk factor associated with weaning protocol is premature weaning trials that can result in $\operatorname{DVWR}(58,73,74)$. Hemodynamic instabilities associated with DVWR include low cardiac output syndrome, pulmonary edema, pulmonary hypertension, respiratory distress, and cardiac arrhythmias $(5,19,53$, $59,68,72,74,75,84,85,107,136,137,166,167)$. Altered fluid balance, which includes increased positive fluid balance and increased chest tube drainage and re-exploration surgery, is associated with DVWR (107).

\section{Pathophysiology of dysfunctional ventilator weaning response: Mechanical} ventilation is an inevitable support for all postoperative cardiac surgery patients to maintain airways and oxygenation during the recovery process. However, early extubation is essential to prevent complications related to mechanical ventilation $(2,40$ $45,51,140,143,152-154)$. Cardiac surgery patients may fail to wean because of various causes related to physiological systems such as neuro, circulatory, respiratory, metabolic, and renal systems. The respiratory causes include impaired respiratory center drive or, more frequently, neuromuscular abnormalities including respiratory muscle fatigue, impaired lung mechanics, or impaired gas exchange capability (58). 
The common cardiopulmonary pathophysiological mechanism involving DVWR is impaired gas exchange. Impaired gas exchange in postoperative heart surgery patients is the result of altered ventilation, altered perfusion, and the mismatch between ventilation and perfusion. The pathophysiological alterations involve alveolar hypoventilation, increased pulmonary shunt, decreased $\mathrm{O}_{2}$ delivery, pulmonary hypertension, pulmonary vasoconstriction, and atlectasis (58). The imbalance between the supply and demand of oxygen is another altered physiology result in DVWR, particularly with open-heart surgery, which involves sternotomy and a considerable amount of compromised respiratory muscle function. An imbalance between respiratory capacity and imposed load, respiratory muscle fatigue, alteration in ventilation, and impaired gas exchange leads to prolonged mechanical ventilation (58). Ventilatory muscle fatigue is associated with altered nutrition, electrolyte imbalances, hormonal imbalances, impaired $\mathrm{O}_{2}$ delivery. Phosphate and magnesium deficiencies are the metabolic causes of weaning failure and prolonged mechanical ventilation (168). One study has reported that maintaining optimum glucose levels has an association with successful weaning (34).

The neurological causes of DVWR include failure in coordination between the brain stem and the cortical chemoreceptive and mechano-receptive feedback mechanism due to electrolyte disturbances, sedation, central apneas, and brain strokes. The failure of peripheral nerves due to metabolic, structural, or drug factors can also result in ventilator dependence.

Cardiovascular causes of DVWR include cardiac decompensation and congestive heart failure resulting in weaning failure. Patients with limited cardiovascular reserve are 
at risk of ischemia or heart failure during weaning resulting from increased circulatory demand. Respiration has a hydraulic influence upon cardiovascular function (84). Mechanical ventilation with positive pressure (MV) reduces left ventricular preload and afterload. The weaning process has a high risk for silent myocardial ischemia among the cardiac risk population during noncardiac surgery postoperative period $(99,107)$. Removal of positive pressure ventilation results in increased venous return due to the sudden increase in negative thoracic pressure. It also increases the left ventricular afterload caused by negative pleural pressure swings $(169,170)$. Lemaire (1993) demonstrated left ventricular dysfunction during the weaning process among cardiac failure and COPD patients. The study demonstrated the increase in PCWP from 8 to 25 mmHg during failed ventilator withdrawal attempts in the above mentioned patients (169, 170). Frazier (2001) reported that hemodynamic instability during weaning from mechanical ventilation is major cause of weaning failure (171). It is imperative to assess the above predisposing risk factors before weaning trials in order to prevent the premature trials and weaning failures $(5,19,53,59,68,72,74,75,84,85,107,136,137$, $166,167)$.

Mechanical ventilation: Mechanical ventilation is an associated concept with DVWR. A mechanical ventilator is a positive pressure-breathing device that can maintain ventilation and oxygen delivery for a prolonged period (58). The general purpose of mechanical ventilation is to correct ventilatory failure and to achieve desired oxygenation of the tissues. It is also used as a supportive therapy for patients under anesthesia during 
the recovery phase. Accordingly, postoperative CABG patients receive mechanical ventilation in ICU during recovery.

Mechanical ventilation has a significant effect on hemodynamic changes, as it is associated with increased positive end expiratory pressure (PEEP). Inappropriate increase in PEEP may result in the reduction of CO through various mechanisms. The altered physiology related to high PEEP includes diminished venous return due to increased intrathoracic pressure; right ventricular dysfunction secondary to an increase in right ventricular afterload and decrease in right ventricular filling; alteration in left ventricular stretch and decreasing stroke volume and diminished cardiac contractility due to the increased release of humoral factors from the lung. The cardiac effects of positive pressure ventilation depend upon the volume status and pulmonary compliances.

The effects of positive pressure ventilation in hemodynamic changes are severe in the presence of hypovolemia and increased lung compliance. No hemodynamic depression is observed in the presence of hypervolemia and stiff lung (58).

Weaning from mechanical ventilation: Weaning from mechanical ventilation is another associated concept with DVWR. Weaning is a gradual withdrawal or cessation of mechanical ventilation (58). Weaning from mechanical ventilation is performed at the earliest possible time consistent with patient safety. Weaning is initiated when the patient is recovering from the acute stage of surgical problems and when the causes of respiratory failure are sufficiently reversed. Success in weaning the patient relies upon the individual's physiological clinical condition rather than a mechanical decisionmaking process. Recovery from cardiac anesthesia is an important element to consider 
before the initiation of the weaning process in patients after CABG surgery. Recent research findings reveal that fast track cardiac anesthesia was found effective in reducing required mechanical ventilation time after surgery, ICU length of stay, and hospital stay and cost $(2,3,17,65,155-165)$. Another researcher reported that fast track anesthesia enhanced early extubation after CABG surgery, prevented pulmonary complications, and expedited early rehabilitation (157).

Traditional criteria for the weaning process include optimum achievements of vital capacity, inspiratory force, respiratory rate, tidal volume, minute ventilation, and arterial blood gases $(20,38,72,172)$. One researcher reported that traditional weaning criteria are not useful in predicting weaning ability, but $\mathrm{RR}$ and tidal volume may be used as valuable predictors (155). Another finding from a comparative study in evaluation of the value of standard and recent indices in predicting successful extubation following prolonged mechanical ventilation revealed that maximal inspiratory pressure may be used as a valuable predictor of weaning out comes (173). Success in the prediction of weaning outcomes depends upon the assessment of the trend of the criteria rather than single measurements (174).

The weaning process has an effect on cardiopulmonary status. Weaning from mechanical ventilation may cause cardiac output changes as well as redistribution of blood flow to the respiratory muscles. A clinical prospective study revealed that there was splanchnic blood flow reduction after extubation (175). Baseline cardiac function influences on weaning after CABG surgery. Consideration of baseline cardiac function may be an important factor in the selection of an appropriate mode of spontaneous ventilation following controlled mechanical ventilation. A comparison of hemodynamic 
changes during the transition from mechanical ventilation to T-piece, pressure support, and continuous positive airway pressure in canines revealed that baseline ventricular function influenced hemodynamic response to the immediate transition from mechanical to spontaneous ventilation. A significant increase in cardiac output in response to T-piece was reported (171). Abnormal cardiopulmonary changes during the weaning process may be an indicator for DVWR. Findings from a study on cardiopulmonary effects of pressure support ventilation revealed a significant increase in RR and tidal volume in patients who are difficult to wean due to respiratory muscle fatigue (176).

Among the comorbid conditions of DVWR, COPD is a common pulmonary condition associated with prolonged mechanical ventilation after CABG surgery (26, 177-180). In contrast, findings from a series of investigations of the changes in respiratory and circulatory functions in COPD patients during sequential invasivenoninvasive mechanical ventilation therapy revealed that the respiratory and circulatory functions of COPD patients remained stable during the weaning process $(181,182)$.

Dysfunctional ventilator weaning response is a time-sensitive event. There is no standard time limit found in the literature for DVWR. Several researchers reported that the majority of the NVWR occurred at 4 to 8 hours after CABG surgery $(51,53,54,59$, 66). Findings from some recent studies reveal that the rate of weaning failure is higher among early weaning trials, which may indicate an association between premature weaning trials and weaning failures $(58,73,74)$.

In health care practices, there is emphasis on the reduction of ICU length of stay and cost after $\mathrm{CABG}$ surgery. Fast track weaning protocols are found to provide cost effective care after CABG surgery. Fast track weaning protocols enhance early 
extubation, resulting in shorter hospital stay and decreased costs in CABG surgery. The new goal of critical care after CABG surgery is to extubate patients within 4 to 6 hours upon arrival in the ICU $(4,5)$.

The practice of a fast track weaning protocol has reduced the required duration of mechanical ventilation after surgery and reduced ICU and hospital length of stay $(41,43$, $44,51,74,140,141,153,183)$. Findings from one research study revealed that a fast track weaning protocol reduced the percentage of patients requiring more than one course of mechanical ventilation during the hospitalization from $33 \%$ to $26 \%(\mathrm{p}=.039)$, a total cost savings of $\$ 3,440,787$ and a decrease in mortality from $32 \%$ to $28 \%(\mathrm{p}=.062)(184)$. Identification of predicting factors for DVWR may help to promote patient safety and quality care.

\section{Conceptual model of predictive association of cardiopulmonary indicators} with dysfunctional ventilator weaning response: The conceptual model depicted in Figure 2.1 is the visual display of the association of risk factors and CPI with DVWR and NVWR after CABG surgery. It shows that cardiac surgery is followed by ventilator weaning in postoperative critical care. Postoperatively, the mechanical ventilation and hemodynamic stabilization therapies help to restore cardiopulmonary function. The outcome of these therapies is either normal ventilator weaning response (NVWR) or DVWR. The above-mentioned outcomes influence CPI. CPI helps to predict NVWR and DVWR, which are associated with the restored cardiopulmonary function and impaired cardiopulmonary function. Changes in CPI precede the outcome, which can be identified at an early stage at bedside. Therefore, the above concept explains the importance of 


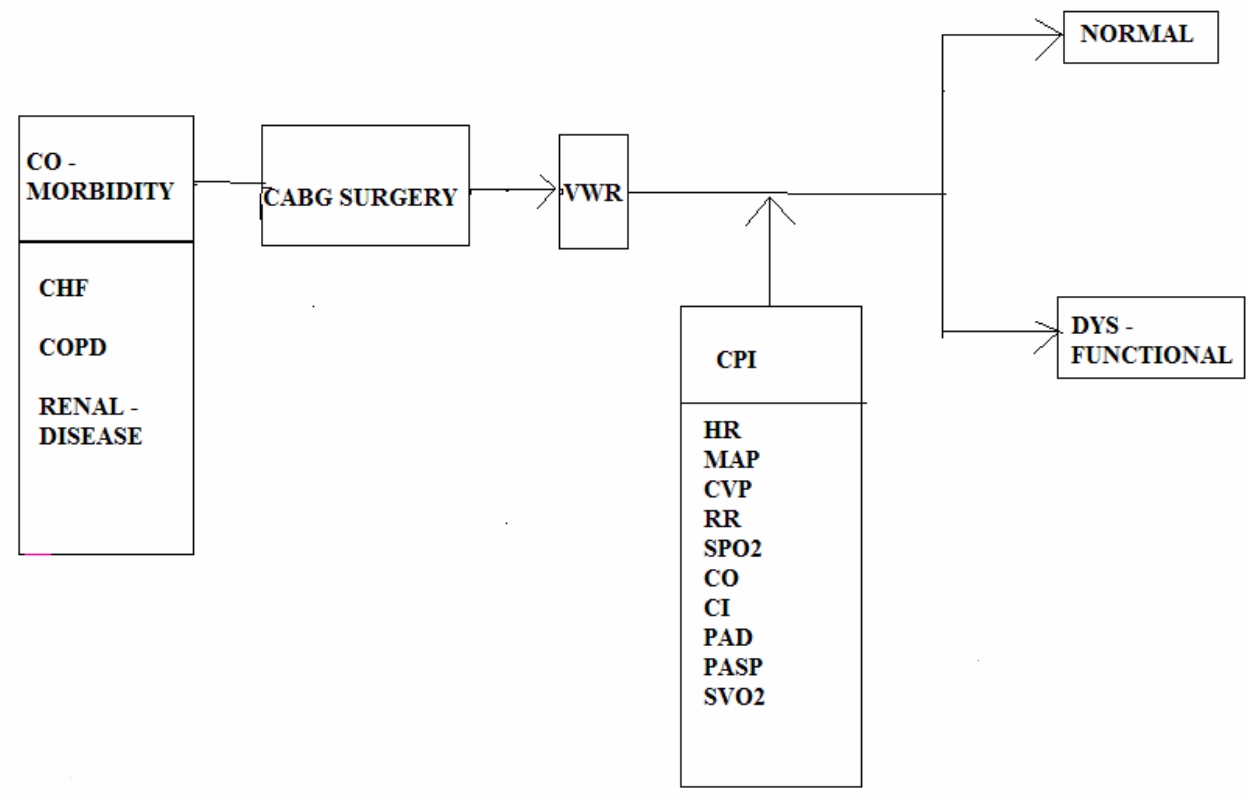

Figure 2.1. Proposed Conceptual Model of Cardiopulmonary Indicators in Predicting Dysfunctional Ventilator Weaning Response after CABG Surgery.

Note.

CHF: congestive heart failure

COPD: chronic obstructive pulmonary disease

CABG: coronary artery bypass surgery

VWR: ventilator weaning response

CPI: cardiopulmonary indicators

HR: heart rate

MAP: mean arterial pressure

CVP: central venous pressure

$\mathrm{RR}$ : respiratory rate

$\mathrm{SPO}_{2 \text { : }}$ oxygen saturation

CO: cardiac output

CI: cardiac index

PAD: pulmonary diastolic pressure

PASP: pulmonary artery systolic pressure

$\mathrm{SVO}_{2}$ : mixed venous oxygen saturation 
determining the temporal association of the CPI with DVWR. Many risk factors are found to be associated with the outcome variable DVWR. Altered cardiopulmonary function following CABG surgery is the precursor for VWR.

The predominant risk factors associated with altered cardiopulmonary function are preoperative comorbid conditions such as increased age $>70$ years, female gender, COPD, $\mathrm{CHF}$, redo surgery, and renal disease. Intraoperative factors such as cardiac surgical techniques, anesthesia, and cardiopulmonary bypass are associated with altered cardiopulmonary function and delayed weaning or DVWR. Postoperative conditions associated with altered cardiopulmonary function include mechanical ventilation, weaning protocols, and interventions for hemodynamic stabilization.

Postoperative recovery of $\mathrm{CABG}$ patients follows two main pathways: restoration of cardiopulmonary function or impaired cardiopulmonary function. Cardiopulmonary function is assessed through the hemodynamic monitoring of CPI. Cardiopulmonary indicators are an index of cardiopulmonary status; they are monitored in the ICU postoperatively through a pulmonary artery catheter, a radial artery catheter, a Spacelabs cardiac monitor, and an Edwards Lifesciences $\mathrm{SVO}_{2}$ monitor. Patients who attain restored cardiopulmonary function manifest optimal cardiopulmonary function and optimal CPI. In contrast, patients who have impaired cardiopulmonary function manifest altered CPI. Alteration of CPI is the predictive sign of DVWR, which can be detected at an early stage.

This study emerges from the belief that these manifestations can be predicted through the assessment of trends of CPI. The outcome of postoperative recovery of CABG surgery patients follows either the NVWR or DVWR pathway. The outcome for 
patients who follow the NVWR pathway is either discharge from the ICU or development of respiratory distress after extubation and reintubation. Those patients who develop respiratory distress after extubation are at risk of developing a complication of PMV. PMV is defined as the condition in which the patient remains on ventilator $>24$ hours after surgery. Patients who exhibit signs of DVWR follow the PMV pathway.

Predictive values of CPI help prevent premature trials and DVWR. Many studies have found an association between some CPI and weaning success. Clinically, CPI is used postoperatively for the titration of the medications, evaluation of the therapy, and assessment of cardiopulmonary function in the ICU. Although the selected CPI for this study have clinical merits, their values in predicting DVWR are unknown. This study evaluates the predictive value of CPI in DVWR with the hypothesis that CPI has predictive value for DVWR. 


\section{CHAPTER 3: METHODOLOGY}

This research study was undertaken with the purpose of describing the characteristics of cardiopulmonary indicators (CPI) among patients with normal ventilator weaning response (NVWR) and dysfunctional ventilator weaning response (DVWR) after coronary artery bypass graft (CABG) Surgery, finding the differences in characteristics of cardiopulmonary indicators between patients with NVWR and DVWR after CABG surgery, and of building a prediction model for DVWR with significant antecedence. This prediction could guide in prevention of DVWR after CABG surgery and in prevention of premature ventilator weaning trials for high-risk patients. This chapter describes the study design, setting, sample, selection of sample, instrumentation, procedure, human subjects, and statistical analysis utilized in this study.

\section{Research Design}

This research study utilized a retrospective case control study with time series design. A hallmark of the case control study is that it begins with people with the disease (cases) and compares them to people without the disease (controls) (185). The cases (DVWR) in this study are defined as patients who remained on a mechanical ventilator $>8$ hours after CABG surgery $(1,6-25)$. The controls (NVWR) in this study were defined as patients who were extubated within 8 hours after CABG surgery $(1,6-25)$. The differences between cases and controls were established in this study by the comparison of CPI measurements between cases and controls on an hourly basis during the postoperative period. The selected CPI data collected hourly in a time series design 
for 12 hours postoperatively in both cases and controls were compared to demonstrate the significant differences. This study design was selected to find the predictive value of CPI for DVWR after CABG surgery.

The independent variable of this study was CPI. The selected CPI to predict DVWR in this research study included heart rate (HR), mean arterial blood pressure (MAP), central venous pressure (CVP), cardiac output $(\mathrm{CO})$, respiratory rate $(\mathrm{RR})$, mixed venous oxygen $\left(\mathrm{SVO}_{2}\right)$, oxygen saturation (54), pulmonary artery diastolic pressure (PAD), and pulmonary artery systolic pressure (PASP). The dependent variable of the study was DVWR, which is operationally defined as the condition in which the patient failed to wean from mechanical ventilation 8 hours after surgery $(17,20,21,55,56)$.

\section{Setting}

The study was conducted at a suburban, 500-bed private for profit Mid-South hospital, with a 40-bed adult ICU. The hospital performs approximately 1 to 3 CABG surgeries daily. In addition, this hospital has a fast track weaning protocol that is implemented after all CABG surgeries. Furthermore, the hospital has an electronic patient file system with electronic documentation and storage of hemodynamic data and patient data for later retrieval.

\section{Selection of Sample}

The study population included all patients who underwent CABG surgery with inclusion criteria guided purposive sampling technique. Three hundred electronic medical records (EPF) of patients who underwent CABG surgery between May 2003 and 
February 2006 and who met the inclusion and exclusion criteria were selected by retrospective sequential sampling. Among the 300 subjects, 100 subjects constituted the case group and 200 subjects constituted the control group. The researcher collected CPI for 12 consecutive hours during postoperative periods, and utilized all 12 hours CPI in descriptive statistics for description of the study sample. Further, the researcher utilized only the first 8 hours data for inferential statistics in accordance with the definition of DVWR.

The source of research data was the EPF system that contains the electronic medical records $(\mathrm{MR})$ of all adult patients who underwent $\mathrm{CABG}$ surgery within the study period. The inclusion criteria were age $>18$ years and patients who had undergone CABG surgery. The exclusion criteria were patients who had any one of the following treatments: intra aortic balloon pump, pacemaker, epinephrine, nor epinephrine, dopamine, and continuous renal replacement therapy (CRRT).

Selection of cases and controls: Cases and controls were selected with operational definitions from the EPF medical records of patients who had undergone CABG surgery. Cases were defined as patients who remained on mechanical ventilator more than 8 hours after CABG surgery $(1,6-25)$. Controls were defined as patients who were extubated within 8 hours after the CABG surgery $(1,6-25)$. Cases and controls were selected from the same hospital.

Sample size and sampling technique: The sample size requirement for fitting a multiple regression analysis was 5 to 10 observations for each potential predicting 
variable. Accordingly, the sample size of the cases was 100. In case control studies, the proportion of the study population that consists of cases is determined by the ratio of controls per case, typically ranging from 1 to 4 controls per case (185-188). This study utilized the 1: 2 ratio of cases and controls. Thus, this study consisted of 100 cases and 200 controls.

\section{Instrument and Measurements}

This case control study utilized the medical records of those patients who had CABG surgery in a Mid-South hospital from 2003 to 2006. Data were abstracted from the EPF medical records system to an Excel spreadsheet on the researcher's personal computer. The EPF system is an electronic recording and storage of the vital parameters measured by various sensors, which are transmitted to a medical service provider via mobile communication. The data was stored in a database through the automatic electronic charting interface from the Spacelab PC I 90303B bedside cardiac monitor to the patient's personal computer, which stored it in an electronic patient file (EPF). The file contained all medical documentation. Authorized clinics and physicians can access this file to support diagnosis and therapy.

The CPI measurements involved hemodynamic monitoring devices such as a Spacelabs cardiac monitor, Edwards Lifesciences cardiac output $\mathrm{SVO}_{2}$ monitor, 7.5 FR pulmonary artery catheters, Servo I mechanical ventilator, and Massimo TM pulse ox sensor. The reliability and quality control tests were performed for the Spacelabs cardiac monitor, Servo I ventilator and Edwards Lifesciences cardiac output monitor by the biomedical department of the hospital. The reliability of the $\mathrm{SVO}_{2}$ monitor was evaluated 
and maintained periodically through performance check procedures by the biomedical department of the hospital. Sensitivity of Spacelabs cardiac monitors were estimated as $92 \%$. The positive predictive value of Spacelabs cardiac monitors were estimated as $97 \%$. The estimated reliability of the oxymetry values for the Massimo SET oxymetry was $95 \%(54)$.

The instrument used for storing the data was a Gateway laptop computer. This computer has high quality compatibility with external equipment. This device has been qualified to the following standards: universal input 10.5VDC to $15 \mathrm{VDC}$, meets FCC/CISPR/VCCI class B EMI (conducted and emitted radio noise), vibration and shock, high efficiency, $100 \%$ HI-POT tested, $100 \%$ cyclic burn-in, humidity, and temperature.

\section{Sources of Data}

The types of data obtained from the EPF system were demographic, clinical, and CPI measurements. The demographic data includes age and sex, which were retrieved from the history and physical pages of the EPF. The clinical data included COPD, CHF, renal disease and redo surgery, which were retrieved from the history and physical pages of the EPF. Extubation time was derived from the respiratory flow sheet and nursing assessment pages of the EPF. The CPI data were HR, MAP, CVP, CO, RR, $\mathrm{SVO}_{2}, \mathrm{SPO}_{2}$, PAD and PASP, which were recorded from the nursing assessment and vital signs confirmation page of the EPF. 


\section{Procedure}

The researcher obtained approval for the research study from the University of Tennessee Health Science Center (UTHSC) IRB and the Mid-South hospital IRB. After IRB approval, the researcher obtained a security pin from the hospital administration to access the medical records of the CABG patients through the EPF system. A list of CABG patients from January 1, 2003 to February 28, 2006 was prepared from the ICU CABG surgery logbook. The medical records of patients who met the inclusion criteria were accessed through the Electronic Patient File (EPF).

\section{Data Analysis}

Statistical analysis: Statistical analysis was performed using a SAS statistical analysis software program. Descriptive statistics were performed on all variables, including demographic and comorbid data. All the statistical significance tests were performed as two-tailed tests. A p-value of $<0.05$ was considered significant.

The selected statistical analysis procedures in this research study included PROC UNIVARIATE, PROC FREQ, PROC GLM, PROC REG, PROC MIXED REPEATED MEASURE ANOVA, and PROC LOGISTIC. Descriptive statistics were derived from a univariate procedure. To compare the means and find the differences in the means between the groups, t-statistics were performed for continuous data, and Chi-square tests were used to compare the difference in proportions of categorical data. A multiple group comparison was performed by repeated measure ANOVA PROC MIXED. To estimate the correlation coefficience for a linear regression model, GLM procedure and REG 
procedures were performed. To build the prediction model, multiple logistic regressions through LOGISTIC procedure were used.

The first aim of this research study was to describe the characteristics of CPI in postoperative CABG patients with DVWR. To address this specific aim, study descriptive statistics were used to aid in describing and summarizing the sample by individual demographic variables, clinical variables, and $\mathrm{CPI}$ in postoperative $\mathrm{CABG}$ patients with DVWR. The procedures used for this purpose were PROC UNIVARIATE, PROC FREQ, unpaired t-test and Chi-square test. The descriptions were presented as frequencies, percentages, means, standard deviations, t-score, p-value, and trend line graphs. To perform the descriptive statistics the patients who were extubated after 12 hours of surgery were grouped as DVWR and coded as 1 . Then the CPI values of this group were analyzed by the SAS program's univariate procedure to explore each variable in a data set separately.

To describe the characteristics of CPI in postoperative CABG patients with NVWR, descriptive statistics were used to aid in describing and summarizing the sample by individual CPI in postoperative CABG patients with NVWR. They included frequencies, percentages, means, standard deviations, and ranges. To perform the descriptive statistics, the patients who were extubated after 8 hours of surgery were grouped as DVWR. Then a SAS program univariate procedure was used to explore each variable in the data set separately and analyzed CPI values for cases and controls. From this analysis, the estimation of sample mean, standard error of the mean, $\mathrm{t}$-score, $\mathrm{p}$-value, and trend graph were generated and presented for individual $\mathrm{CPI}$ in postoperative $\mathrm{CABG}$ patients with DVWR. 
The second aim of this research study was to find the differences in the characteristics of CPI between postoperative CABG patients with DVWR and NVWR. The samples of DVWR and NVWR were independent, and the data points in one group were unrelated to the data points in the other group. Therefore, to address this specific aim of the research study, the researcher performed an unpaired t-test to find the differences in means of the individual CPI between the two groups. To perform the unpaired $t$ - test, the $t$-test procedure from a SAS program was used. The t-test procedure by default examined the hypothesis of equal variances and provides t-statistics for CPI in postoperative CABG patients with DVWR and NVWR. From this procedure, means of the samples, standard deviations of the samples, pooled estimates of the standard deviations, sizes of the samples, degrees of freedom $(\mathrm{n} 1+\mathrm{n} 2-2)$, t-statistics values, and p-values were generated. Next, the researcher tested whether the population mean of the first sample was the same as or different from the mean of the second sample. A null and alternative hypothesis was formulated. Then, the pooled variance estimation was computed using a weighted average of the individual sample variances.

Next, the differences in the distribution of demographic variables and comorbidities were assessed through a PROC FREQ Chi-square test. Further, the comparison between cases and controls, including CPI and demographic variables and comorbidities, was made through PROC MIXED REPEATED MEASURE ANOVA.

The third aim of this research study was to build a prediction model for DVWR among postoperative $\mathrm{CABG}$ patients with significant antecedence. To address this specific aim, the researcher utilized the multiple logistic regression model, including all the possible CPI and confounding variables. To perform the multiple logistic 
regressions, the researcher used the LOGISTIC procedure in a SAS program. The goal of logistic regression was to find the best fitting model to describe the relationship between the dichotomous characteristic of interest, which is DVWR, and a set of independent predicting variables. Logistic regression generates the coefficients (and its standard errors and significance levels) of a formula to predict a logit transformation of the probability of the presence of the characteristic of interest:

$$
\operatorname{logit}(\mathrm{p})=\mathrm{b}_{0}+\mathrm{b}_{1} \mathrm{x}_{1}+\mathrm{b}_{2} \mathrm{x}_{2}+\mathrm{b}_{3} \mathrm{x}_{3}+\ldots+\mathrm{b}_{\mathrm{k}} \mathrm{x}_{\mathrm{k}}
$$

where $\mathrm{p}$ was the probability of presence of the characteristic of interest. The logit transformation is defined as the logged odds:

Odds $=\mathrm{P} / 1-\mathrm{P}=$ Probability of presence of characteristic/Probability of absence of characteristic; and $\operatorname{logit}(\mathrm{p})=\ln (\mathrm{p} / 1-\mathrm{p})$.

Parameter estimation in a logistic regression chose the parameters that maximized the likelihood of observing the sample values. From this procedure, the maximum likelihood estimation of DVWR was estimated as odds after transforming the dependent into a logit variable (the natural log of the odds of the dependent occurring or not). In this way, logistic regression estimates the probability of a DVWR occurring. A stepwise procedure with the combination of forward selection and backward elimination was performed for all the CPI and possible confounding factors.

Testing overall model fitness: The null model- 2 Log Likelihood is given by -2 * $\ln \left(\mathrm{L}_{0}\right)$ where $\mathrm{L}_{0}$ is the likelihood of obtaining the observations if the independent variables had no effect on the outcome. The full model-2 Log Likelihood is given by -2 * 
$\ln (\mathrm{L})$ where $\mathrm{L}$ is the likelihood of obtaining the observations with all independent variables incorporated in the model. The difference between these two yields a Chisquare statistic, which is a measure of how well the independent variables affect the outcome or dependent variable. If the p-value for the overall model fit statistic is less than the conventional 0.05 , then there is evidence that at least one of the independent variables contributes to the prediction of the outcome.

Regression coefficients: The regression coefficients are the coefficients $b_{0}, b_{1}$, $b_{2}, \ldots b_{k}$ of the regression equation:

$$
\operatorname{logit}(p)=b_{0}+b_{1} x_{1}+b_{2} x_{2}+b_{3} x_{3}+\ldots+b_{k} x_{k}
$$

An independent variable with a regression coefficient not significantly different from $0(\mathrm{P}>0.05)$ can be removed from the regression model. If $\mathrm{P}<0.05$, then the variable contributes significantly to the prediction of the outcome variable. The logistic regression coefficients show the change (increase when $b_{i}>0$, decrease when $b_{i}<0$ ) in the predicted log odds of having the characteristic of interest for a one-unit change in the independent variables. Odd ratios with $95 \%$ CI were computed by taking the exponential of both sides of the regression equation as given above; the equation can be rewritten as:

$$
\text { odds }=\frac{p}{1-p}=e^{b_{0}} \times e^{b_{1} x_{1}} \times e^{b_{2} x_{2}} \times e^{b_{3} x_{3}} \times \ldots \times e^{b_{k} x_{k}}
$$

The next step was to assess the effectiveness of the model by a classification table. The classification table is used to evaluate the predictive accuracy of the logistic 
regression model. In this table, the observed values for the dependent outcome and the predicted values (at a cut-off value of $p=0.30$ ) were cross-classified.

\section{Quality Control}

The accuracy of data collection was monitored by the researcher and by the committee director, Dr. Carol Thompson. The collected data were verified against the medicals record by the Mid-South hospital informatics.

\section{Data Management and Safety Monitoring Plan}

This research study did not involve risk to the human subjects, but it was responsibility for maintaining Health Insurance Portability and Accountability Act (HIPAA) policy. To maintain HIPAA policy, the collected data were stored in the researcher's personal computer, and the confidentiality of the data was maintained by coding the data. The assigned code and subjects' identification were stored in a secured system by the researcher. In addition, the sponsor, Dr. Carol Thompso, monitored the

data biweekly. The dissertation committee monitored the research protocol and progress of the study.

\section{Protection of Human Subjects}

This research study utilized pre-existing data from the medical records of all patients who underwent coronary artery bypass graft $(\mathrm{CABG})$ surgery in the selected Mid-South hospital. Although this research study did not involve risk to the subjects, it had an inherent responsibility to comply with the Health Insurance Portability and 
Accountability Act (HIPAA) policy. The researcher observed the standards recommended by the Health Insurance Portability and Accountability Act of 1996 (HIPAA) and the Mid-South hospital policy in protecting the identifiers of protected health information (PHI).

In addition, the researcher followed the HIPAA policy for the Mid-South hospital in accessing, storing, handling, and sharing the data. For the purposes of maintaining the privacy of individual patients, the researcher assigned a code for all individual cases and restricted access to the researcher's computer systems using passwords. All personal identifiers were removed from the data to protect confidentiality of the subjects. A master key that linked to the PHI and the code number was maintained in a separate and secured location, and access was strictly restricted by using computer passwords. Individual subjects will not be identified in any presentations or publications based on the results of this research study.

All variables in the extracted data that reveal the identity of individuals were removed prior to entering data in the researcher's extraction form in the computer. The research record and data were labeled with code numbers.

The code and definitions of code were stored in the Mid-South hospital computer system to ensure the protection of subjects' confidentiality with the adherence to the HIPAA policy of the Mid-South hospital. The access to the research data was strictly controlled by the researcher through a designated security PIN. The computer was secured and maintained locked with a password. Printed materials that contained research data were shredded as per the Mid-South hospital policy. 


\section{CHAPTER 4: RESULTS}

A retrospective case control study was conducted to determine the predictive values of cardiopulmonary indicators $(\mathrm{CPI})$ in the prediction of dysfunctional ventilator weaning response (DVWR) after coronary artery bypass graft (CABG) surgery. The purposes of the study were to describe the characteristics of CPI among patients with normal ventilator weaning response (NVWR) and DVWR after CABG surgery, to find the differences in characteristics of cardiopulmonary indicators between patients with NVWR and DVWR after CABG surgery, and to build a prediction model for DVWR. To accomplish the purposes of the research study, a case control study with time series design was adopted. The CPI measurements were collected on an hourly basis for 12 hours postoperatively for cases and controls through the retrospective electronic patient file system audit. The data were entered in the data extraction Excel spreadsheet and imported into SAS for statistical analysis. The inferential statistics included preplanned comparisons between cases and controls involving study variable CPI.

\section{Sample Description}

Three hundred electronic medical records of patients who underwent CABG surgery between May 2003 and February 2006 and who met the inclusion and exclusion criteria were selected. Among the 300 subjects, 100 subjects constituted the case group and 200 subjects constituted the control group. One subject in the case group was excluded from data analysis due to extreme missing data. As a result, the total study sample consisted of 99 cases and 200 controls. The researcher collected data for 12 
consecutive postoperative periods, and utilized all 12 hours of data for descriptive statistics in description of the study sample. Further, the researcher utilized only the first 8 hours data for inferential statistics in accordance with the definition of DVWR.

To describe the characteristics of CPI in patients with DVWR and NVWR after CABG surgery, descriptive statistics were used for individual demographic variables and CPI in both case and control groups. PROC UNIVARIATE and PROC FREQ procedures were used to describe all variables. To perform the descriptive statistics, the patients who were extubated within 8 hours of surgery were grouped as NVWR (controls) and the patients who were extubated after 8 hours were grouped as DVWR (cases). The demographic variable age was divided into three groups as group 1 (33-52 years), group 2 (53-72 years), and group 3 (73-92 years) for the purpose of description.

The SAS program PROC FREQ, which analyzes each variable in a data set separately, was used to analyze the demographic variables of both groups. A summary of frequencies and percentages of demographic variables are presented in Table 4.1. Next, demographic variables and CPI values were analyzed by a univariate procedure. From this procedure the mean and standard deviation for each individual variable was generated and will be presented in table form later. The hourly means of the CPI were plotted in line graphs for both cases and controls to depict and described the trend of the variables.

Age was included in the analysis as actual numbers and for the purpose of meaningful description the descriptive findings were presented as three groups, which included group 1 (33-52 years), group 2 (53-72 years), and group 3 (73-92 years). 
Table 4.1. Frequency and Percentage of Subjects in Demographic Categories.

\begin{tabular}{lcc}
\hline Demographic Category & $\begin{array}{c}\text { Controls }(\mathrm{n}=200) \\
\text { Frequency/Percentage }\end{array}$ & $\begin{array}{c}\text { Cases }(\mathrm{n}=99) \\
\text { Frequency/ Percentage }\end{array}$ \\
\hline Age (Years) & & \\
$33-52$ & $45(22.5 \%)$ & $17(17.17 \%)$ \\
$53-72$ & $120(60.00 \%)$ & $53(53.54 \%)$ \\
$73-92$ & $35(17.50 \%)$ & $29(29.29 \%)$ \\
& & \\
Sex & & $58(58.59 \%)$ \\
Male & $146(73 \%)$ & $41(41.41 \%)$ \\
Female & $54(27 \%)$ & \\
Comorbidities & & $21(21.21 \%)$ \\
COPD & $10(5 \%)$ & $12(12.12 \%)$ \\
CHF & $4(2 \%)$ & $4(4.04 \%)$ \\
Renal failure & $4(2 \%)$ &
\end{tabular}

Note.

COPD: chronic obstructive pulmonary disease

CHF: congestive heart failure 
The study sample consisted of subjects who ranged in age from 33 years to 91 years. The mean age of the subjects in the case group was $64.28 \pm 11.45$ years. The mean age of the subjects in the control group was $61.8 \pm 11.05$ years.

The majority of the study sample consisted of 204 male subjects (68.23\%); the number of female patients was 95 (31.77\%). The case group consisted of 58 (58.59\%) males, and the control group consisted of $146(73 \%)$ males.

Comorbid conditions that were included in this study were COPD, CHF, and renal disease. The total number of subjects who had comorbid conditions was $55(18.39 \%)$. The subjects who had COPD constituted the majority at 31 (10.37\%); the number of CHF subjects was $16(5.35 \%)$, and the number of renal failures was $8(2.68 \%)$.

Number of grafts: The number of grafts ranged from 1 to 6 . The number of subjects who had one graft in cases was $6(6.06 \%)$, and in controls $7(3.52 \%)$. The number of subjects who had two grafts in cases was 13 (13.13\%), and in controls 38 (38.38\%). The number of subjects who had three grafts in cases was 38 (38.38\%), and in controls 75 (37.69\%). The number of subjects who had four grafts in cases was 30 $(30.30 \%)$ and in controls $53(26.63 \%)$. The number of subjects who had five grafts in cases was $10(10.10 \%)$, and in controls $20(10.05 \%)$. The number of subjects who had 6 grafts in cases was $2(2.02 \%)$ and in controls $6(3.02 \%)$. In this study, the majority of the subjects had three grafts ( $38 \%$ in cases and controls). 
The total number of hours in ventilation: The range of the total ventilator hours in cases was 8.30 to 349 hours and in controls 1.35 to 8 hours. The average hours in ventilation in the control group was $4.51 \pm 1.58$ hours.

Body surface area: Body surface area (BSA) was estimated from the $\mathrm{CO}$ and $\mathrm{CI}$ values, as this data was not available. Although BSA was calibrated in the $\mathrm{SVO}_{2}$ monitor, it was not recorded in the nursing notes. Mean BSA in the case group was 1.96, with a standard deviation of 0.249. Mean BSA in the control group was 2.028, with a standard deviation of 0.245 .

Post operative ventilator settings and medications: The ventilator setting was common for all the patients during the weaning process. The setting was SIMV mode ventilation with $\mathrm{FIO}_{2} 40 \%$, pressure support of $10 \mathrm{~cm}$ of $\mathrm{H}_{2} \mathrm{O}$, PEEP of $5 \mathrm{~cm}$ of $\mathrm{H}_{2} \mathrm{O}$. The use of sedation was common for all patients in the study sample. The choice of sedation was precedex continuous infusion and morphine whenever necessary. The postoperative use of dobutamine to titrate for cardiac index of 2 liters was common for all the subjects. The vasodilator nipride was used as the PRN drug of choice to treat hypertension in all the subjects. The cardiac monitors, monitoring techniques, and weaning protocol were common for both case and control groups.

\section{Results of Cardiopulmonary Indicators}

After coronary artery bypass surgery, the first research question of this research study was: What are the characteristics of cardiopulmonary indicators in patients with 
dysfunctional ventilator weaning response and normal ventilator weaning response? Cardiopulmonary indicators values were analyzed by a univariate procedure. From this procedure the mean and standard deviation for each individual variable was generated. The hourly means of the CPI were plotted in line graphs for both cases and controls to depict and describe the trend of the variables.

Heart rate: The average heart rate during the first 8 postoperative hours for cases was 94.67 per minute and for controls was 93.64 per minute. The hourly mean and standard deviation for cases and controls are presented in Table 4.2. The mean HR ranged from 93.01 to 96.73 in cases and from 90.84 to 95.01 in controls. The mean heart rate trend showed that the heart rate was higher in cases during the first 3 hours (Figure 4.1). An upward tend was noted in both cases and controls during the first 3 hours. In addition, a downward trend occurred in mean heart rate during fifth, sixth, seventh and eighth hours among cases, while there was a plateau in controls.

Mean arterial pressure: The average MAP during the first 8 postoperative hours for cases was $78.28 \mathrm{~mm} / \mathrm{Hg}$ and for controls $79.59 \mathrm{~mm} / \mathrm{Hg}$. The hourly means and standard deviations for cases and controls are presented in Table 4.3. The trend of mean MAP showed that there was a downward trend in MAP from first hour to sixth hour in both case and control groups, MAP stabilized at seventh and eighth hour (Figure 4.2). Overall, MAP in the control group was higher than in the case group. 
Table 4.2. Means and Standard Deviations of Heart Rate (HR) for Cases and Controls.

\begin{tabular}{ccc}
\hline Hour & $\begin{array}{c}\text { Cases } \\
\text { Mean \& Standard } \\
\text { Deviation }\end{array}$ & $\begin{array}{c}\text { Controls } \\
\text { Mean \& Standard } \\
\text { Deviation }\end{array}$ \\
\hline 1 & $94.24(16.11)$ & $90.84(13.89)$ \\
2 & $96.42(17.84)$ & $93.03(14.05)$ \\
3 & $96.73(15.93)$ & $94.13(13.84)$ \\
4 & $94.27(14.78)$ & $95.01(13.73)$ \\
5 & $95.37(15.13)$ & $94.39(13.96)$ \\
6 & $93.85(13.91)$ & $94.59(13.55)$ \\
7 & $93.01(14.62)$ & $94.31(15.02)$ \\
8 & $93.45(14.20)$ & $92.78(13.59)$ \\
9 & $92.37(13.8)$ & $92.51(12.82)$ \\
10 & $92.57(14.34)$ & $92.10(13.01)$ \\
11 & $90.85(13.02)$ & $90.41(12.85)$ \\
12 & $91.48(11.73)$ & $91.72(12.7)$ \\
\hline
\end{tabular}

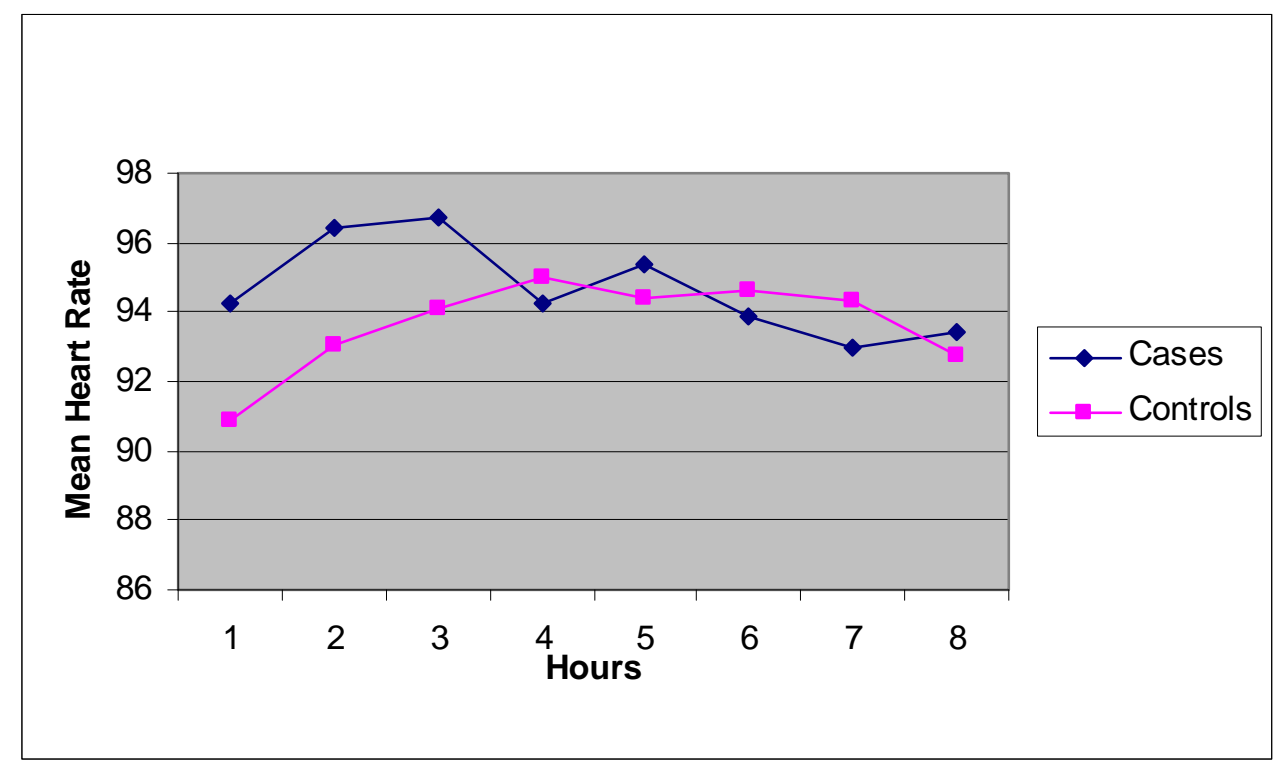

Figure 4.1. Mean Heart Rate Trend. 
Table 4.3. Means and Standard Deviations of Mean Arterial Pressure for Cases and Controls.

\begin{tabular}{ccc}
\hline Hour & $\begin{array}{c}\text { Cases } \\
\text { Mean \& Standard } \\
\text { Deviation }\end{array}$ & $\begin{array}{c}\text { Controls } \\
\text { Mean \& Standard } \\
\text { Deviation }\end{array}$ \\
\hline 1 & $82.64(13.89)$ & $83.90(12.48)$ \\
2 & $81.20(13.44)$ & $79.76(11.9)$ \\
3 & $79.19(10.71)$ & $79.92(10.24)$ \\
4 & $77.97(11.25)$ & $80.41(10.76)$ \\
5 & $76.43(12.13)$ & $79.75(10.44)$ \\
6 & $77.15(13.53)$ & $79.10(10.34)$ \\
7 & $75.77(11.15)$ & $77.32(9.61)$ \\
8 & $75.91(13.52)$ & $76.59(10.82)$ \\
9 & $75.21(10.28)$ & $74.68(7.92)$ \\
10 & $73.62(9.26)$ & $75.06(8.85)$ \\
11 & $75.12(10.42)$ & $75.64(8.66)$ \\
12 & $76(10.07)$ & $74.83(7.11)$ \\
\hline
\end{tabular}

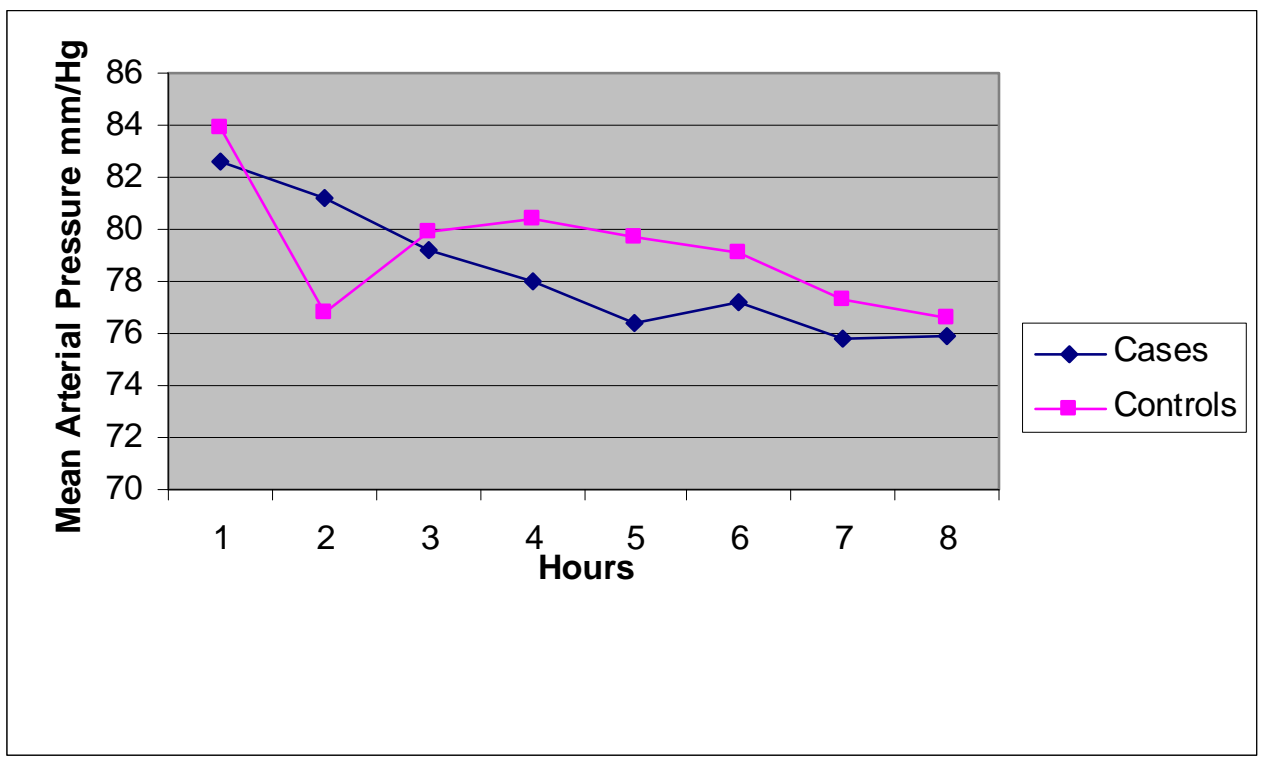

Figure 4.2. Mean Arterial Pressure Trend. 
Central venous pressure: The average CVP during the first 8 postoperative hours for cases was $10.75 \mathrm{~cm} / \mathrm{H}_{2} \mathrm{O}$ and for controls $10.32 \mathrm{~cm} / \mathrm{H}_{2} \mathrm{O}$. The hourly means and standard deviations for cases and controls are presented in Table 4.4. The trend of CVP showed that CVP in cases was lower in first hour than CVP in controls (Figure 4.3). However, CVP in cases showed an upward trend from second hour. The overall trend showed that CVP in cases was higher than in controls from second to eighth hour.

Respiratory rate: Average RR during the first 8 postoperative hours for cases was 14.47 per minute and for controls 16.96 per minute. The hourly means and standard deviations for cases and controls are presented in Table 4.5. The trend of RR showed that there was an upward trend from the first hour to the eighth hour in both cases and controls. The overall respiratory rate was higher in controls than cases (Figure 4.4).

Oxygen saturation: The average $\mathrm{SPO}_{2}$ during the first 8 postoperative hours for cases was $98.29 \%$ and $98 \%$ for controls. The hourly means and standard deviations for cases and controls are presented in Table 4.6. The trend showed that the $\mathrm{SPO}_{2}$ of the cases was higher than controls for the first 3 hours, but there was a downward trend in cases from fourth hour. The trend of $\mathrm{SPO}_{2}$ in controls showed stability for first 3 hours and a downward trend at fourth hour, followed by a stable $\mathrm{SPO}_{2}$ for the next 4 hours. Overall, the $\mathrm{SPO}_{2}$ showed a downward trend at the fourth hour in both cases and controls. Although the $\mathrm{SPO}_{2}$ of cases was higher than controls, the $\mathrm{SPO}_{2}$ of controls showed a more stable trend than the cases (Figure 4.5). 
Table 4.4. Means and Standard Deviations of Central Venous Pressure for Cases and Controls.

\begin{tabular}{ccc}
\hline Hour & $\begin{array}{c}\text { Cases } \\
\text { Mean \& Standard } \\
\text { Deviation }\end{array}$ & $\begin{array}{c}\text { Controls } \\
\text { Mean \& Standard } \\
\text { Deviation }\end{array}$ \\
\hline 1 & $10.47(3.90)$ & $12.03(4.68)$ \\
2 & $10.85(3.77)$ & $10.29(3.73)$ \\
3 & $10.87(3.71)$ & $10.14(3.60)$ \\
4 & $11.20(3.81)$ & $10.58(3.72)$ \\
5 & $10.96(3.48)$ & $10.58(3.60)$ \\
6 & $10.44(3.62)$ & $9.92(2.69)$ \\
7 & $10.59(4.55)$ & $9.46(3.67)$ \\
8 & $10.61(4.24)$ & $9.56(3.97)$ \\
9 & $10.70(2.8)$ & $9.28(2.53)$ \\
10 & $10.27(2.67)$ & $9.63(2.76)$ \\
11 & $10.09(2.58)$ & $9.68(2.66)$ \\
12 & $11.1(2.87)$ & $10.01(2.65)$ \\
\hline
\end{tabular}

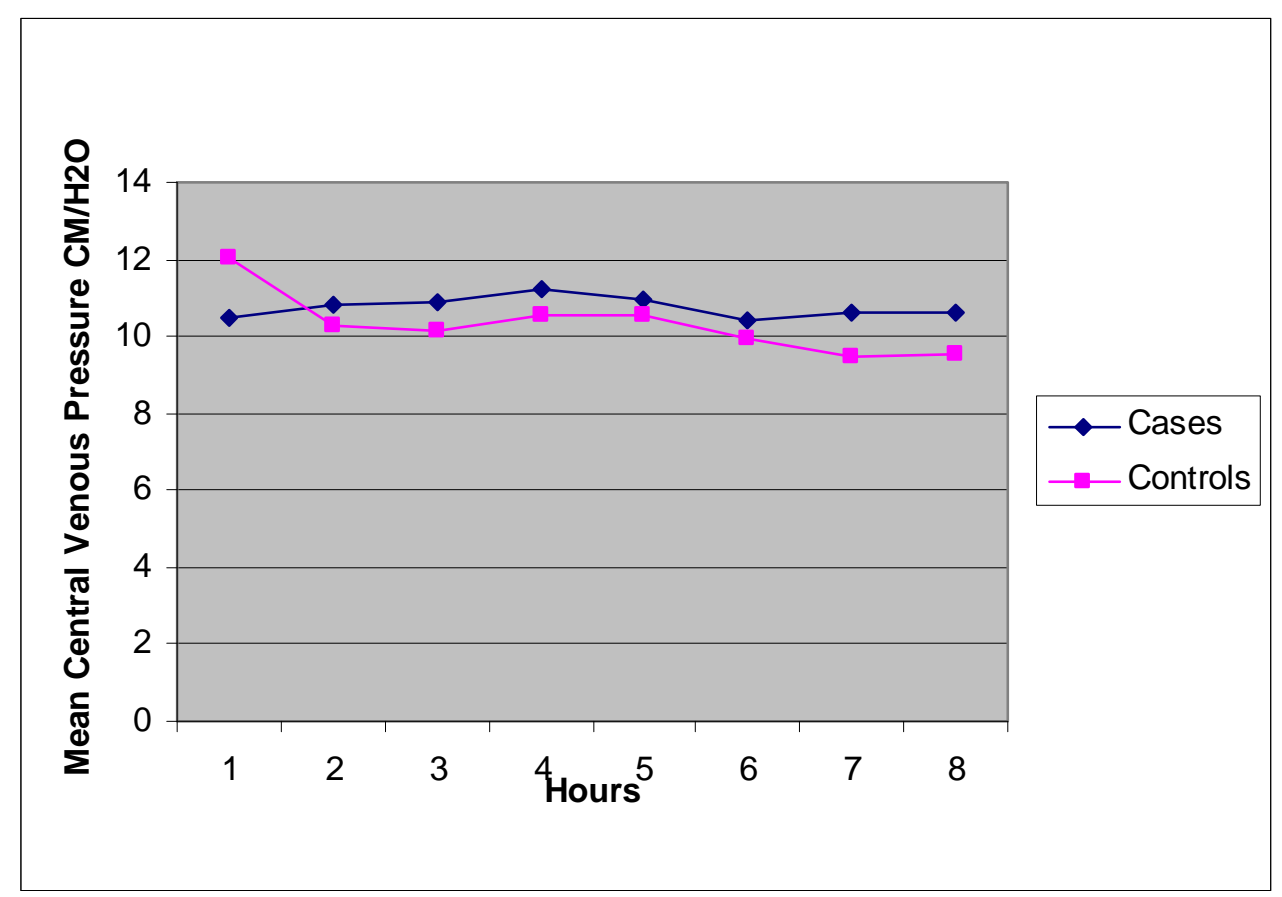

Figure 4.3. Mean Central Venous Pressure Trend. 
Table 4.5. Means and Standard Deviations of Respiratory Rate for Cases and Controls.

\begin{tabular}{ccc}
\hline Hour & $\begin{array}{c}\text { Cases } \\
\text { Mean \& Standard } \\
\text { Deviation }\end{array}$ & $\begin{array}{c}\text { Controls } \\
\text { Mean \& Standard } \\
\text { Deviation }\end{array}$ \\
\hline 1 & $12.20(4.73)$ & $12.94(4.61)$ \\
2 & $13.31(5.16)$ & $15.19(5.82)$ \\
3 & $14.25(5.62)$ & $16.16(5.16)$ \\
4 & $14.83(5.87)$ & $17.03(4.98)$ \\
5 & $15.08(5.24)$ & $17.58(4.61)$ \\
6 & $15.51(5.67)$ & $18.67(4.49)$ \\
7 & $15.45(5.99)$ & $18.96(4.17)$ \\
8 & $15.16(5.41)$ & $19.14(4.01)$ \\
9 & $15.57(4.69)$ & $19.12(3.47)$ \\
10 & $16.5(5.42)$ & $19.09(3.17)$ \\
11 & $16.78(4.25)$ & $19.19(3.04)$ \\
12 & $17.28(4.69)$ & $18.92(2.71)$ \\
\hline
\end{tabular}

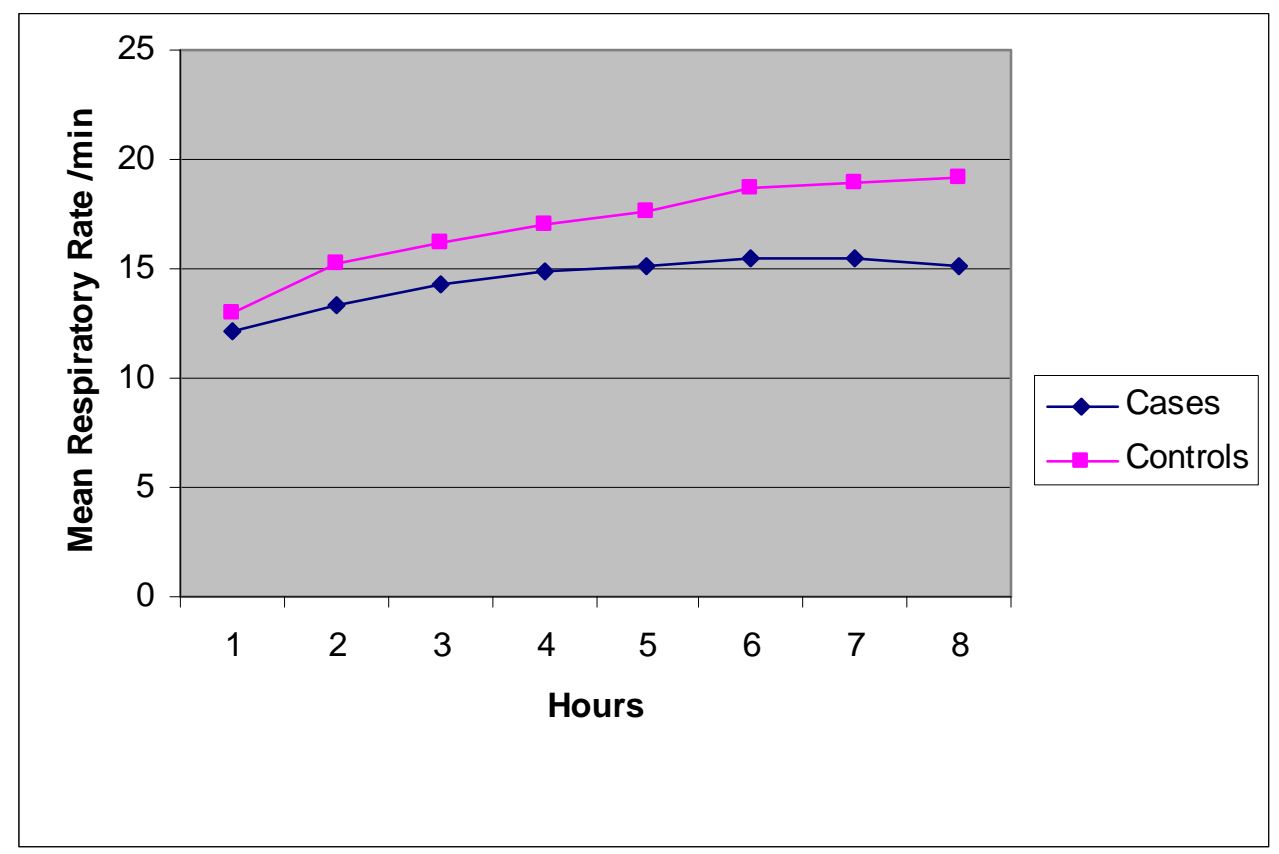

Figure 4.4. Mean Respiratory Rate Trend. 
Table 4.6. Means and Standard Deviations of Oxygen Saturation for Cases and Controls.

\begin{tabular}{ccc}
\hline Hour & $\begin{array}{c}\text { Cases } \\
\text { Mean \& Standard } \\
\text { Deviation }\end{array}$ & $\begin{array}{c}\text { Controls } \\
\text { Mean \& Standard } \\
\text { Deviation }\end{array}$ \\
\hline 1 & $98.54(2.10)$ & $98.25(2.33)$ \\
2 & $98.24(2.10)$ & $98.17(2.33)$ \\
3 & $98.60(1.89)$ & $98.21(2.36)$ \\
4 & $98.71(1.91)$ & $98.07(2.28)$ \\
5 & $98.35(2.05)$ & $97.65(2.38)$ \\
6 & $98.18(2.50)$ & $97.64(2.27)$ \\
7 & $97.87(2.93)$ & $97.74(2.15)$ \\
8 & $97.82(2.38)$ & $97.50(2.39)$ \\
9 & $97.79(2.49)$ & $96.78(2.99)$ \\
10 & $97.21(2.99)$ & $96.81(2.29)$ \\
11 & $97.04(2.38)$ & $97.19(2.94)$ \\
12 & $97.62(2.41)$ & $97.39(2.27)$ \\
\hline
\end{tabular}

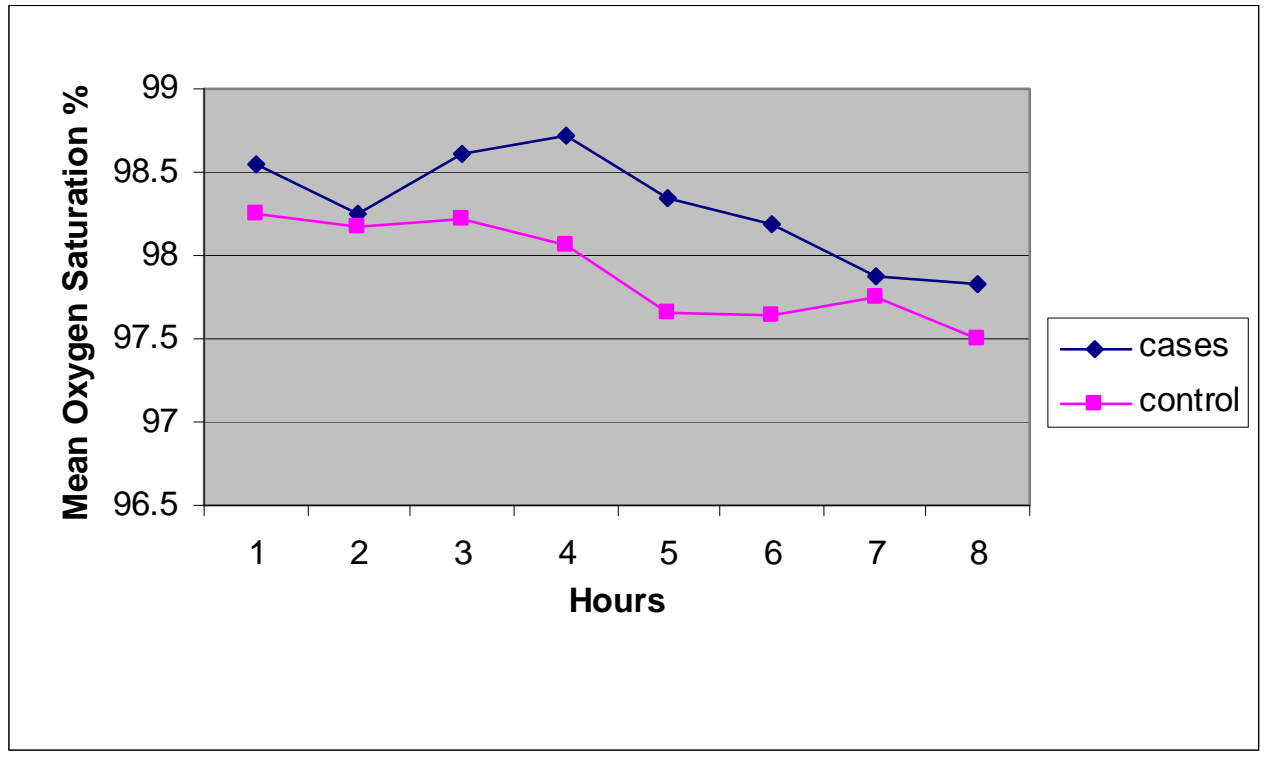

Figure 4.5. Mean Oxygen Saturation Trend. 
Cardiac output: The average CO during first 8 postoperative hours for cases was 5.2 liter per minute and for controls was 5.7 liter per minute. The hourly mean and standard deviations for cases and controls are presented in Table 4.7. The CO means ranged from 5.07 to 5.50 in cases and 5.37 to 6.04 in controls. The trend of $\mathrm{CO}$ showed that there was an upward trend in both cases and controls from first hour to sixth hour and a plateau at seventh and eighth hour. The mean $\mathrm{CO}$ of controls was higher than cases throughout the trend (Figure 4.6).

Cardiac index: The average $\mathrm{CI}$ during the first 8 postoperative hours for cases was 2.6 and 2.85 liters per minute for controls. The hourly means and standard deviations for cases and controls are presented in Table 4.8. The mean CI ranged from 2.53 to 2.78 liters per minute in cases and 2.64 to 2.96 liters per minute in controls. The trend of CI showed that there was an upward trend in both cases and controls from the first hour to the sixth hour and a downward trend at the seventh and eighth hour in controls. In cases, there was a plateau in the seventh and eight hours. The mean CI of controls was higher than cases throughout the trend (Figure 4.7).

Pulmonary artery diastolic pressure: The average PAD during the first 8 postoperative hours for cases was $16.66 \mathrm{~mm} / \mathrm{Hg}$ and for controls $13.34 \mathrm{~mm} / \mathrm{Hg}$. The hourly means and standard deviations for cases and controls are presented in Table 4.9. Figure 4.8 shows the trend of PAD means for DVWR (cases) and NVWR (controls). The trend reveals that the mean PAD pressure was the same for cases and controls during the first hour. However, the mean PAD pressure for the cases remained higher than for the 
Table 4.7. Means and Standard Deviations of Cardiac Output for Cases and Controls.

\begin{tabular}{ccc}
\hline Hour & $\begin{array}{c}\text { Cases } \\
\text { Mean \& Standard } \\
\text { Deviation }\end{array}$ & $\begin{array}{c}\text { Controls } \\
\text { Mean \& Standard } \\
\text { Deviation }\end{array}$ \\
\hline 1 & $5.08(1.28)$ & $5.37(1.23)$ \\
2 & $5.07(1.56)$ & $5.45(1.31)$ \\
3 & $5.20(1.48)$ & $5.59(1.34)$ \\
4 & $5.21(1.46)$ & $5.68(1.26)$ \\
5 & $5.32(1.62)$ & $5.6(1.25)$ \\
6 & $5.34(1.30)$ & $6.04(1.50)$ \\
7 & $5.50(1.40)$ & $5.86(1.31)$ \\
8 & $5.46(1.40)$ & $5.89(1.24)$ \\
9 & $5.54(1.26)$ & $5.74(1.16)$ \\
10 & $5.53(1.19)$ & $5.74(1.04)$ \\
11 & $5.56(1.23)$ & $5.71(1.12)$ \\
12 & $5.53(1.26)$ & $6.11(1.15)$ \\
\hline
\end{tabular}

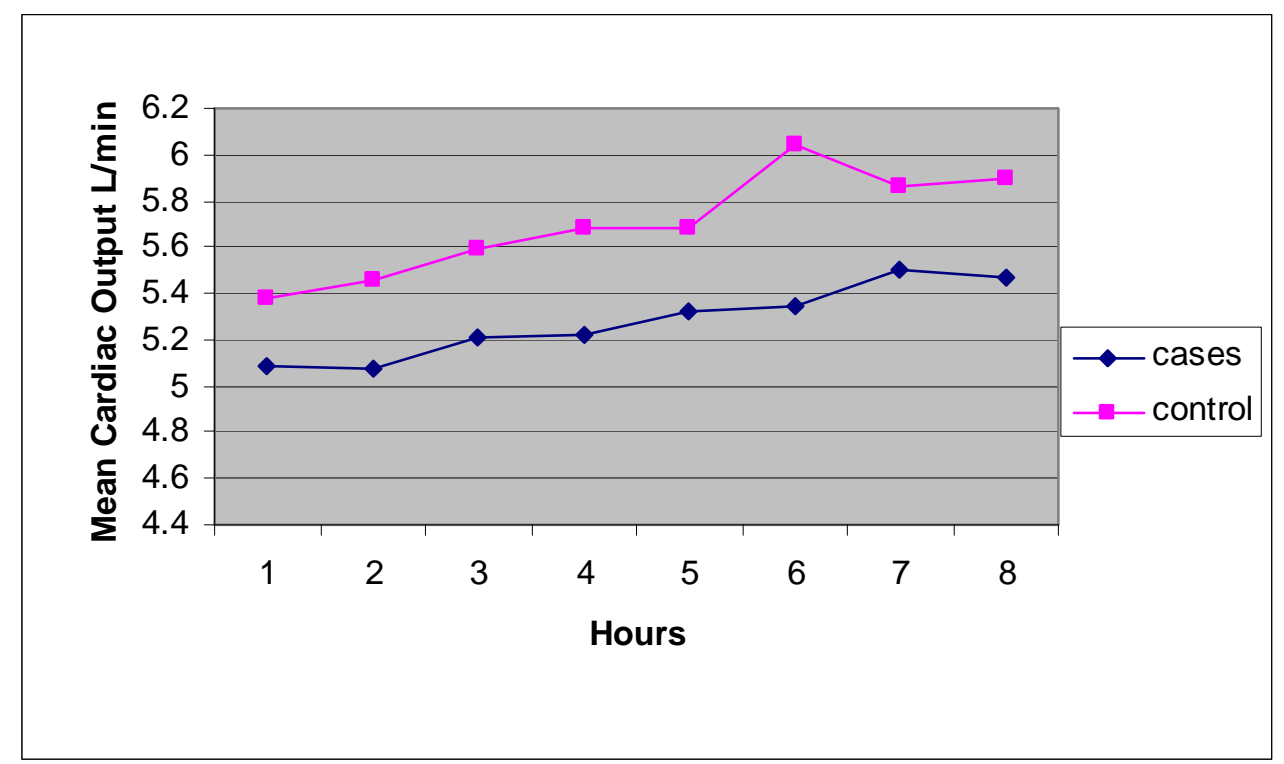

Figure 4.6. Mean Cardiac Output Trend. 
Table 4.8. Means and Standard Deviations of Cardiac Index for Cases and Controls.

\begin{tabular}{ccc}
\hline Hour & $\begin{array}{c}\text { Cases } \\
\text { Mean \& Standard } \\
\text { Deviation }\end{array}$ & $\begin{array}{c}\text { Controls } \\
\text { Mean \& Standard } \\
\text { Deviation }\end{array}$ \\
\hline 1 & $2.59(0.6)$ & $2.64(0.59)$ \\
2 & $2.53(0.6)$ & $2.68(0.6)$ \\
3 & $2.63(0.67)$ & $2.76(0.62)$ \\
4 & $2.67(0.64)$ & $2.79(0.56)$ \\
5 & $2.70(0.68)$ & $2.80(0.59)$ \\
6 & $2.72(0.57)$ & $2.9(0.67)$ \\
7 & $2.78(0.53)$ & $2.89(0.50)$ \\
8 & $2.76(0.61)$ & $2.93(0.53)$ \\
9 & $2.72(0.5)$ & $2.84(0.53)$ \\
10 & $2.79(0.63)$ & $2.85(0.50)$ \\
11 & $2.82(0.50)$ & $2.81(0.51)$ \\
12 & $2.87(0.62)$ & $3.03(0.54)$ \\
\hline
\end{tabular}

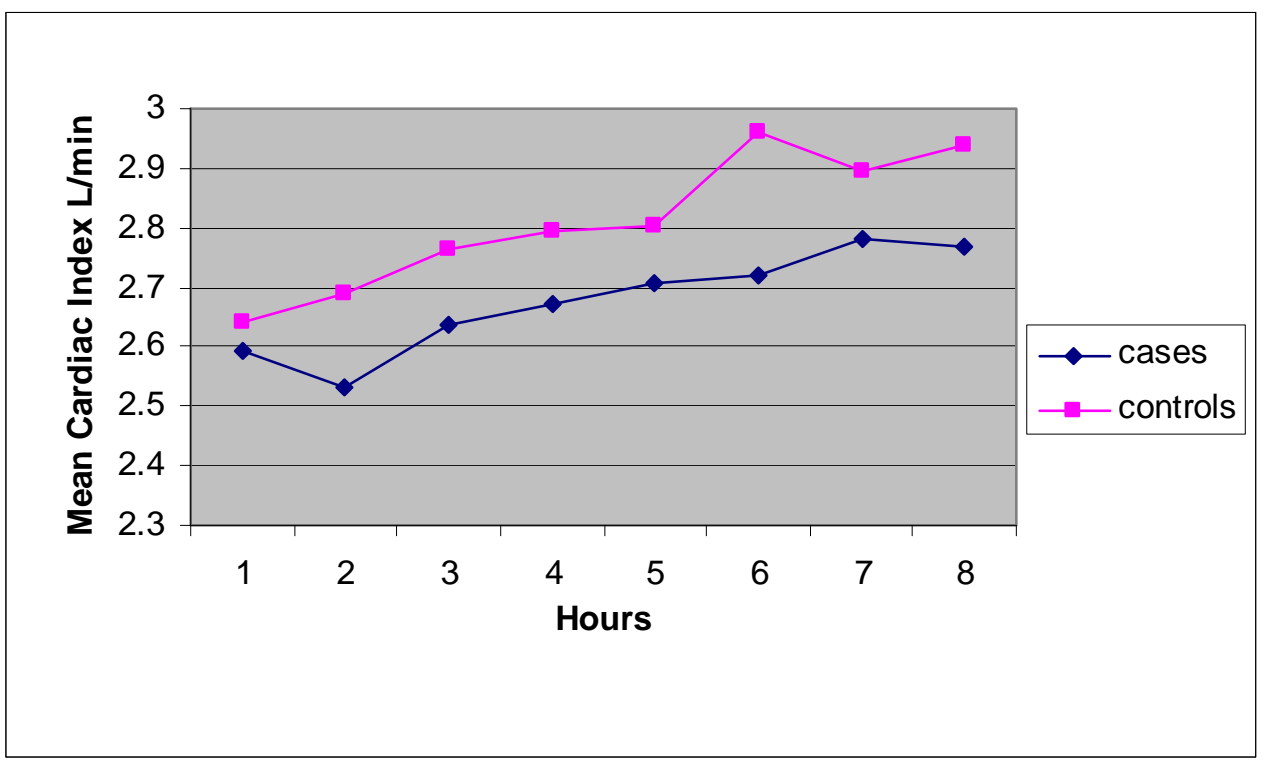

Figure 4.7. Mean Cardiac Index Trend. 
Table 4.9. Means and Standard Deviation Pulmonary Artery Diastolic Pressure for Cases and Controls.

\begin{tabular}{ccc}
\hline Hour & $\begin{array}{c}\text { Cases } \\
\text { Mean \& Standard } \\
\text { Deviation }\end{array}$ & $\begin{array}{c}\text { Controls } \\
\text { Mean \& Standard } \\
\text { Deviation }\end{array}$ \\
\hline 1 & $15.06(5.52)$ & $14.63(5.08)$ \\
2 & $15.32(4.7)$ & $14.47(5.23)$ \\
3 & $15.72(4.87)$ & $14.34(5.0)$ \\
4 & $15.60(4.62)$ & $13.74(4.53)$ \\
5 & $15.44(4.65)$ & $13.04(4.62)$ \\
6 & $15.45(4.89)$ & $12.40(4.44)$ \\
7 & $14.43(4.69)$ & $12.07(4.57)$ \\
8 & $26.26(4.8)$ & $12.03(4.72)$ \\
9 & $14.27(4.81)$ & $12.46(4.8)$ \\
10 & $13.81(4.76)$ & $11.63(4.15)$ \\
11 & $13.88(4.02)$ & $12.70(4.34)$ \\
12 & $14.38(4.92)$ & $12.59(4.16)$ \\
\hline
\end{tabular}

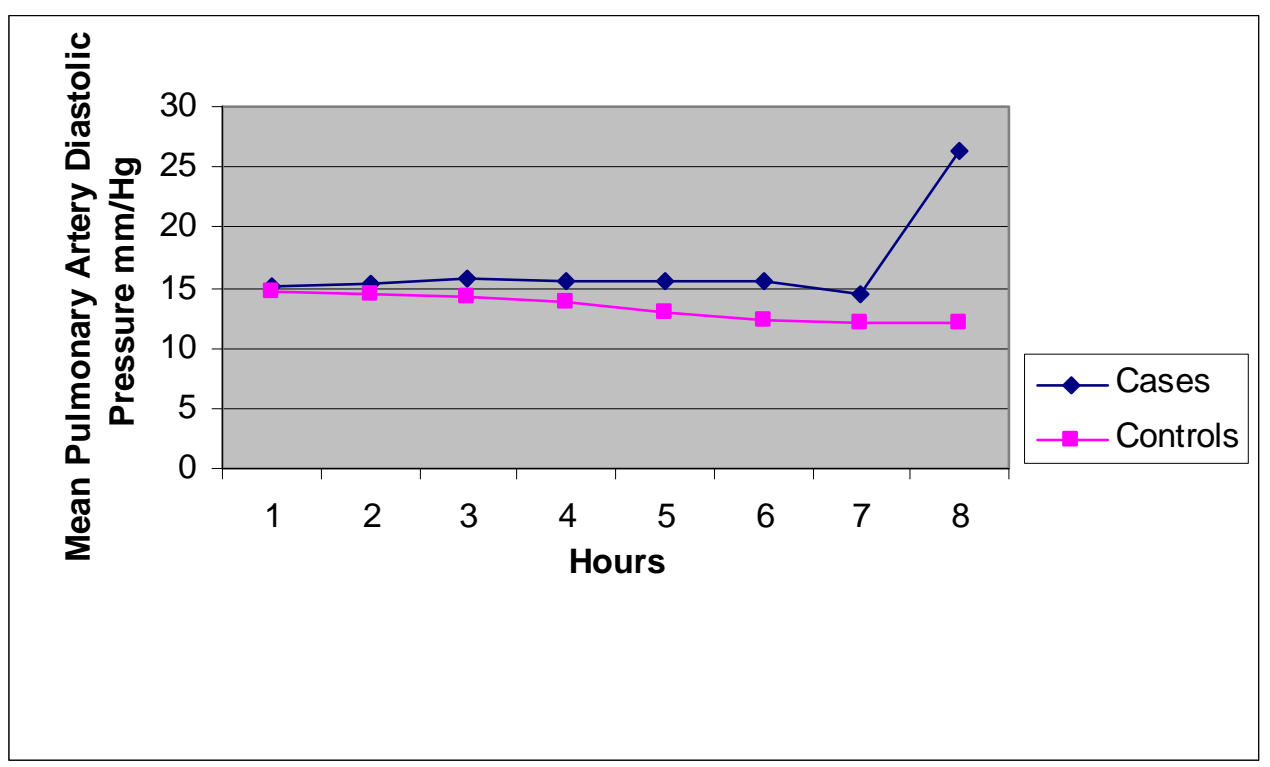

Figure 4.8. Pulmonary Artery Diastolic Pressure Trend. 
control group throughout 8 hours, while the mean PAD of controls decreased during the third, fourth, fifth, and sixth hours, followed by a plateau at the seventh and eighth hours.

Pulmonary artery systolic pressure: Average PASP during first 8 postoperative hours for cases was $29.2 \mathrm{~mm} / \mathrm{Hg}$ and for controls $28 \mathrm{~mm} / \mathrm{Hg}$. The hourly mean and standard deviations for cases and controls are presented in Table 4.10. Figure 4.9 shows the trend of mean PASP. It reveals that mean PASP in patients with DVWR (cases) was higher than in patients with NVWR (control) for the first 3 hours. There was a gradual increase in PASP in patients with NVWR from the fourth hour, and it remained higher than in the cases from the fourth to the eighth hours.

Mixed venous oxygen saturation: The average $\mathrm{SVO}_{2}$ during first 8 postoperative hours for cases was $69 \%$ and for controls $68 \%$. The hourly mean and standard deviations for cases and controls are presented in Table 4.11. The mean $\mathrm{SVO}_{2}$ ranged from $68 \%$ to $69.63 \%$ in cases and from $67.81 \%$ to $69.00 \%$ in controls. The mean $\mathrm{SVO}_{2}$ in cases was higher than in controls (Figure 4.10). There was a downward trend in the first 3 hours in cases, followed by an upward trend during the fourth, fifth, and sixth hours and a decline in the seventh hour, followed by an upward trend in the eighth hour. In controls, there was a downward trend during the first 2 hours and an upward trend during the third and fourth hour, followed by a downward trend during the fifth and sixth hours and a plateau during the seventh and eighth hours. 
Table 4.10. Means and Standard Deviations of Pulmonary Artery Systolic Pressure for Cases and Controls.

\begin{tabular}{ccc}
\hline Hour & $\begin{array}{c}\text { Cases } \\
\text { Mean \& Standard } \\
\text { Deviation }\end{array}$ & $\begin{array}{c}\text { Controls } \\
\text { Mean \& Standard } \\
\text { Deviation }\end{array}$ \\
\hline 1 & $28.65(8.06)$ & $27.69(8.45)$ \\
2 & $28.90(8.10)$ & $28.19(7.5)$ \\
3 & $29.65(7.69)$ & $29.02(7.57)$ \\
4 & $29.34(7.93)$ & $29.32(7.61)$ \\
5 & $29.30(7.14)$ & $29.84(7.8)$ \\
6 & $29.77(7.69)$ & $29.86(7.63)$ \\
7 & $29.04(7.63)$ & $29.10(7.39)$ \\
8 & $28.98(8.03)$ & $29.28(8.18)$ \\
9 & $30.16(8.08)$ & $29.61(8.61)$ \\
10 & $29.77(8.28)$ & $28.42(7.55)$ \\
11 & $29.64(7.56)$ & $29.59(7.28)$ \\
12 & $30.27(7.53)$ & $30.28(7.30)$ \\
\hline
\end{tabular}

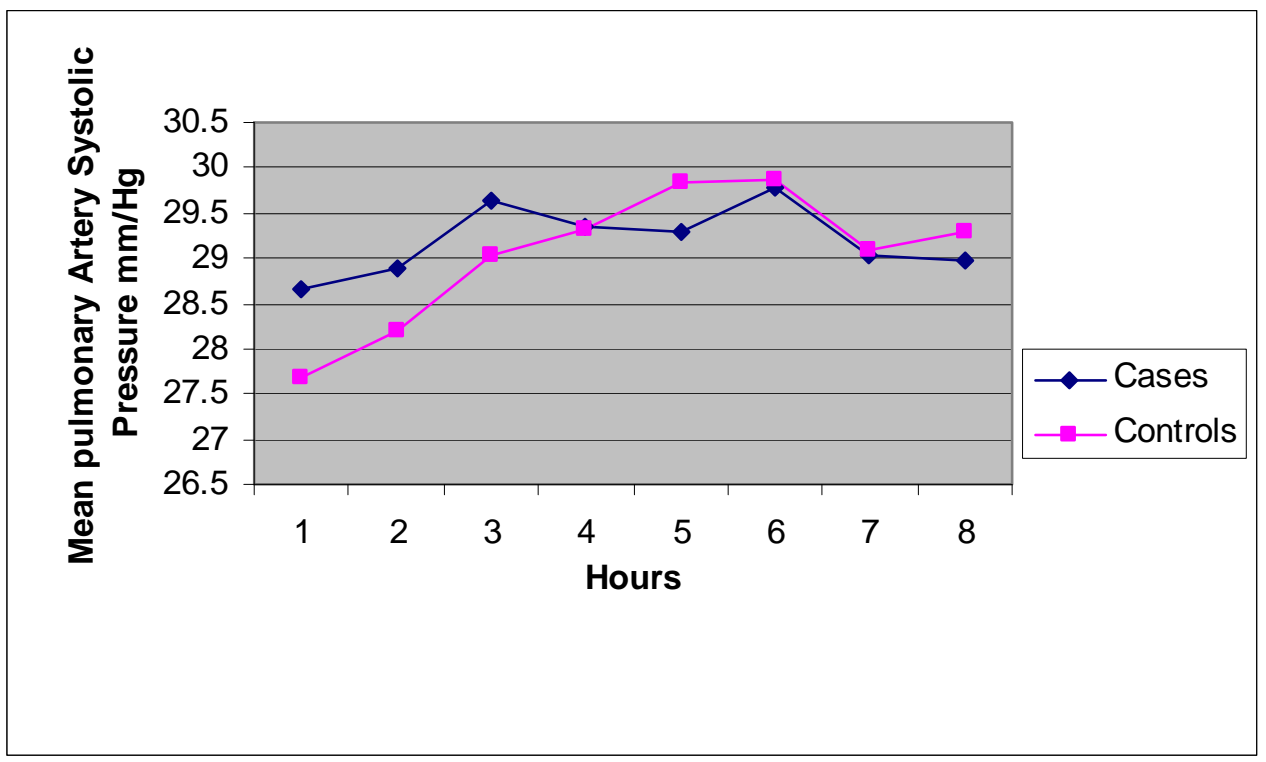

Figure 4.9. Mean Pulmonary Artery Systolic Pressure Trend. 
Table 4.11. Means and Standard Deviations of Mixed Venous Oxygen Saturation for Cases and Controls.

\begin{tabular}{ccc}
\hline Hour & $\begin{array}{c}\text { Cases } \\
\text { Mean \& Standard } \\
\text { Deviation }\end{array}$ & $\begin{array}{c}\text { Controls } \\
\text { Mean \& Standard } \\
\text { Deviation }\end{array}$ \\
\hline 1 & $69.56(7.91)$ & $69.00(7.50)$ \\
2 & $68.69(6.44)$ & $68.01(7.64)$ \\
3 & $68.04(7.34)$ & $68.34(7.13)$ \\
4 & $68.25(7.29)$ & $68.40(6.81)$ \\
5 & $69.26(7.12)$ & $68.16(7.14)$ \\
6 & $69.15(7.28)$ & $67.81(7.04)$ \\
7 & $68.46(7.09)$ & $68.32(6.19)$ \\
8 & $69.63(6.54)$ & $68.29(6.21)$ \\
9 & $68.9(6.26)$ & $68.45(6.38)$ \\
10 & $69.65(5.96)$ & $68.84(7.15)$ \\
11 & $70.58(5.37)$ & $67.97(6.07)$ \\
12 & $69.33(6.33)$ & $67.66(5.37)$ \\
\hline
\end{tabular}

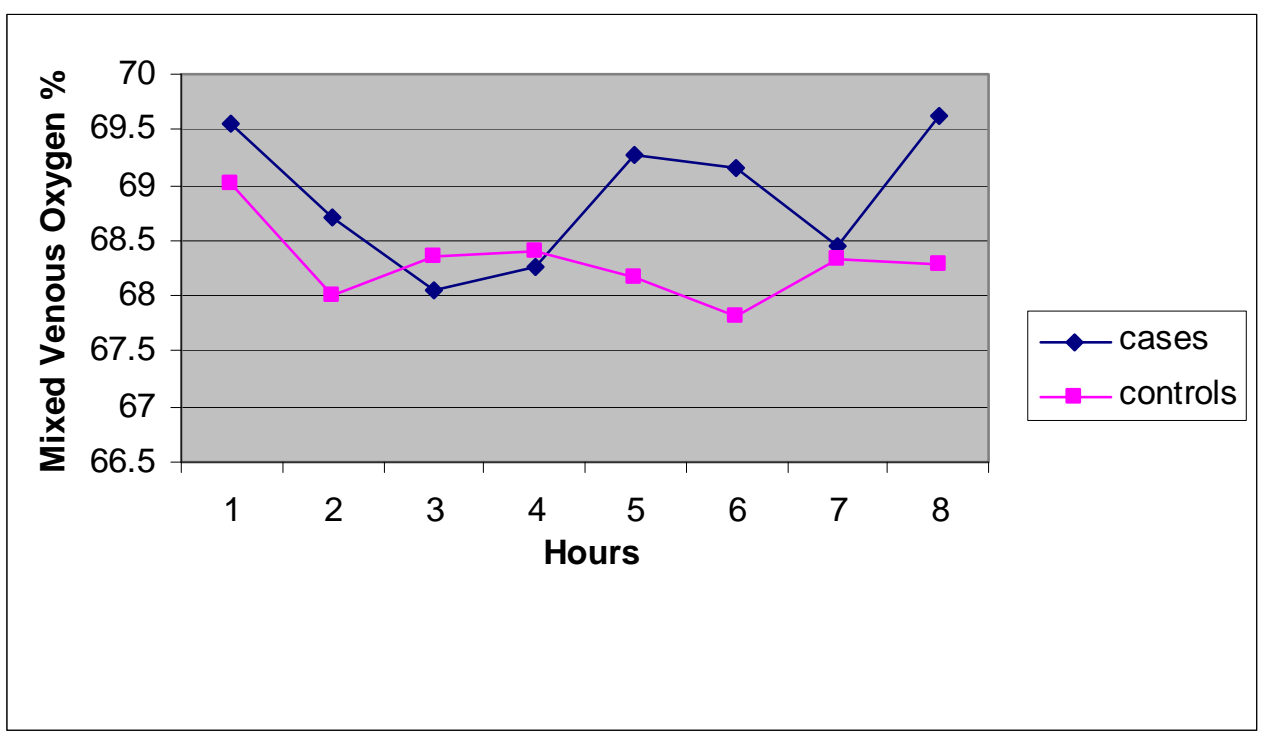

Figure 4.10. Mean Mixed Venous Oxygen Trend. 


\section{Differences in the Characteristics of Cardiopulmonary Indicators}

The second research question of this research study was: In patients after coronary artery bypass surgery, what are the differences in the characteristics of cardiopulmonary indicators in patients with dysfunctional ventilator weaning response and normal ventilator weaning response?

To answer the above research question, the researcher found the differences in the characteristics of CPI between DVWR (cases) and NVWR (controls) by performing an unpaired t-test. The results are displayed in tables later.

The differences in the mean heart rate were established by performing an unpaired $\mathrm{t}$-test comparing the hourly mean of heart rate in cases and controls. The result is displayed in Table 4.12. The result revealed that there were no significant differences in the hourly mean heart rate between cases and controls except at the first hour $(p=0.05)$.

Differences in the mean MAP were established by performing an unpaired t-test comparing the hourly mean of MAP in case and control groups. The results are displayed in Table 4.13 and revealed no significant differences in the hourly mean MAP between cases and controls except at the fifth hour $(\mathrm{p}=0.01)$.

The differences in the mean CVP were established by performing an unpaired t-test comparing the hourly mean of the CVP in cases and controls. The result displayed in Table 4.14 revealed that there were no significant differences between cases and controls in the hourly mean CVP of first, second, fourth, fifth, and sixth hours. However, there was a significant difference between cases and controls in the mean CVP at the third hour $(\mathrm{p}=0.05)$, the seventh hour $(\mathrm{p}=0.004)$, and the eighth hour $(\mathrm{p}=0.01)$. 
Table 4.12. Differences between Cases and Controls in Mean Heart Rate per Minute.

\begin{tabular}{ccc}
\hline Hour & $\begin{array}{c}\text { Differences in } \\
\text { Mean Heart Rate } \\
\text { (Unpaired t-test) }\end{array}$ & P-Value \\
\hline 1 & -1.89 & $0.05^{*}$ \\
2 & -1.66 & 0.09 \\
3 & -1.46 & 0.14 \\
4 & 0.43 & 0.66 \\
5 & -0.56 & 0.57 \\
6 & 0.45 & 0.65 \\
7 & 0.73 & 0.46 \\
8 & -0.41 & 0.68 \\
\hline
\end{tabular}

Note. $^{*}=$ Statistically significant

Table 4.13. Differences between Cases and Controls in Mean Arterial Pressure in $\mathrm{mm} / \mathrm{Hg}$.

\begin{tabular}{ccc}
\hline Hour & $\begin{array}{c}\text { Differences in } \\
\text { Mean Arterial } \\
\text { Pressure } \\
\text { (Unpaired t-test) }\end{array}$ & P-Value \\
\hline 1 & 0.82 & 0.41 \\
2 & 0.94 & 0.33 \\
3 & 0.58 & 0.55 \\
4 & 1.88 & 0.06 \\
5 & 2.58 & $0.01^{*}$ \\
6 & 1.44 & 0.15 \\
7 & 1.31 & 0.16 \\
8 & 0.52 & 0.06 \\
\hline
\end{tabular}

Note. $^{*}=$ Statistically significant 
Table 4.14. Differences between Cases and Controls in Mean Central Venous Pressure in $\mathrm{cm} / \mathrm{H}_{2} \mathrm{O}$.

\begin{tabular}{ccl}
\hline Hour & $\begin{array}{c}\text { Differences in } \\
\text { Mean Central } \\
\text { Venous Pressure } \\
\text { (Unpaired t-test) }\end{array}$ & P-Value \\
\hline 1 & 1.77 & 0.07 \\
2 & -1.37 & 0.17 \\
3 & -1.9 & $0.05^{*}$ \\
4 & -1.55 & 0.12 \\
5 & -0.99 & 0.32 \\
6 & -1.34 & 0.18 \\
7 & -2.88 & $0.04^{*}$ \\
8 & -2.59 & $0.01^{*}$ \\
\hline
\end{tabular}

Note. $*=$ Statistically significant 
The differences in the mean RR were established by performing an unpaired t-test comparing the hourly mean of RR in cases and controls. The result revealed no significant difference in the mean RR between cases and controls at the first hour. However, there was a significant difference between cases and controls in mean RR at all the times (Table 4.15).

Differences in the mean $\mathrm{SPO}_{2}$ were established by performing an unpaired t-test comparing the hourly means of $\mathrm{SPO}_{2}$ in cases and controls. The result displayed in Table 4.16 revealed no significant difference in the hourly mean of $\mathrm{SPO}_{2}$ between cases and controls except in the fourth, fifth, and sixth hour.

The differences in the mean $\mathrm{CO}$ were established by performing an unpaired t-test comparing the hourly mean of $\mathrm{CO}$ in cases and controls. The result is displayed in Table 4.17. The result revealed that there was significant difference in the hourly mean of CO between cases and controls.

The differences in the mean CI were established by performing an unpaired t-test comparing the hourly mean of $\mathrm{CI}$ in cases and controls. The result displayed in Table 4.18 revealed significant difference in the hourly mean of CI between cases and controls in the first, fifth, sixth, and eighth hour.

The differences in the mean PAD were established by performing an unpaired t-test comparing the hourly mean of PAD in cases and controls. The result displayed in Table 4.19 a revealed that there was significant difference in the hourly mean of PAD between cases and controls in all the hours except the first and eighth hour.

The differences in the mean PASP were established by performing an unpaired t-test comparing the hourly mean of PASP in cases and controls. The result displayed in 
Table 4.15. Differences between Cases and Controls in Mean Respiratory Rate per Minute.

\begin{tabular}{ccc}
\hline Hour & $\begin{array}{c}\text { Differences in Mean } \\
\text { Respiratory Rate } \\
\text { (Unpaired t-test) }\end{array}$ & P-Value \\
\hline 1 & 1.3 & 0.19 \\
2 & 2.75 & $0.006^{*}$ \\
3 & 3.01 & $0.002^{*}$ \\
4 & 3.49 & $0.006^{*}$ \\
5 & 4.44 & $<0.0001^{*}$ \\
6 & 4.26 & $<0.0001^{*}$ \\
7 & 6.69 & $<0.0001^{*}$ \\
8 & 7.97 & $<0.0001^{*}$ \\
\hline
\end{tabular}

Note. ${ }^{*}=$ Statistically significant

Table 4.16. Differences between Cases and Controls in Mean Oxygen Saturation \%.

\begin{tabular}{ccc}
\hline Hour & $\begin{array}{c}\text { Differences in } \\
\text { Mean Oxygen } \\
\text { Saturation } \\
\text { (Unpaired t-test) }\end{array}$ & P-Value \\
\hline 1 & -0.45 & 0.65 \\
2 & -0.27 & 0.78 \\
3 & -1.44 & 0.15 \\
4 & -2.43 & $0.01^{*}$ \\
5 & -2.48 & $0.01^{*}$ \\
6 & -1.89 & $0.05^{*}$ \\
7 & -0.43 & 0.66 \\
8 & -1.11 & 0.26 \\
\hline
\end{tabular}

Note. ${ }^{*}=$ Statistically significant 
Table 4.17. Differences between Cases and Controls in Mean Cardiac Output Liters per Minute.

\begin{tabular}{ccc}
\hline Hour & $\begin{array}{c}\text { Differences in Mean } \\
\text { Cardiac Output } \\
\text { (Unpaired t - test) }\end{array}$ & P-Value \\
\hline 1 & 1.96 & $0.02^{*}$ \\
2 & 2.25 & $0.01^{*}$ \\
3 & 2.34 & $0.003^{*}$ \\
4 & 2.99 & $0.02^{*}$ \\
5 & 2.19 & $0.04^{*}$ \\
6 & 4.26 & $0.001^{*}$ \\
7 & 2.52 & $0.01^{*}$ \\
8 & 3.01 & $0.002^{*}$ \\
\hline
\end{tabular}

Note. $^{*}=$ Statistically significant

Table 4.18. Differences between Cases and Controls in Mean Cardiac Index Liters per Minute.

\begin{tabular}{ccl}
\hline Hour & $\begin{array}{c}\text { Differences in Mean } \\
\text { Cardiac Index } \\
\text { (Unpaired t-test) }\end{array}$ & P-Value \\
\hline 1 & 2.12 & $0.03^{*}$ \\
2 & 1.64 & 0.10 \\
3 & 1.67 & 0.09 \\
4 & 1.59 & 0.20 \\
5 & 1.27 & $0.002^{*}$ \\
6 & 3.11 & $0.002^{*}$ \\
7 & 1.77 & 0.07 \\
8 & 2.45 & $0.01^{*}$ \\
\hline
\end{tabular}

Note. $^{*}=$ Statistically significant 
Table 4.19. Differences between Cases and Controls in Mean Pulmonary Artery Diastolic Pressure in $\mathbf{m m} / \mathbf{H g}$.

\begin{tabular}{ccc}
\hline Hour & $\begin{array}{c}\text { Differences in Mean } \\
\text { Pulmonary Artery } \\
\text { Diastolic Pressure } \\
\text { (Unpaired t-test) }\end{array}$ & P-Value \\
\hline 1 & -0.67 & 0.17 \\
2 & -1.36 & $0.02^{*}$ \\
3 & -2.26 & $0.001^{*}$ \\
4 & -3.30 & $<0.0001^{*}$ \\
5 & -4.20 & $<0.0001^{*}$ \\
6 & -5.42 & $<0.0001^{*}$ \\
7 & -4.17 & $<0.0001^{*}$ \\
8 & -1.82 & 0.06 \\
\hline
\end{tabular}

Note. $*=$ Statistically significant 
Table 4.20 revealed no significant difference between cases and controls in the hourly mean of PASP.

The differences in the mean $\mathrm{SVO}_{2}$ were established by performing an unpaired t-test comparing the hourly means of $\mathrm{SVO}_{2}$ in cases and controls. The result displayed in Table 4.21 revealed no significant difference between cases and controls in the hourly mean of $\mathrm{SvO} 2$.

In summary, all the cardiopulmonary indicators showed significant differences between cases and controls except pulmonary artery systolic pressure (Figure 4.11). The earliest differences were noted as early as first hour in heart rate, cardiac output, cardiac index, and pulmonary artery diastolic pressure. The CPI that showed consistent differences included CVP, RR, CO, CI, and PAD.

\section{Prediction Model}

The third research question of this research study was: What is the best predictive model for DVWR after CABG surgery? To build the prediction model, a multiple logistic regression analysis was performed using a logistic procedure with stepwise selection

method. The first consideration in this procedure was multicollinearity. Multicollinearity can be a problem in logistic regression models because of strong correlations between independent variables, which can inflate the variances of the parameter estimates. Therefore, it is imperative to control this problem. In order to identify the multicollinearity of the CPI, a diagnostic procedure was performed by a linear regression analysis through REG procedure with option VIF TOL in SAS. Through this procedure, tolerance and inflation factors were examined for each explanatory variable. First, the 
Table 4.20. Differences between Cases and Controls in Mean Pulmonary Artery Systolic Pressure in $\mathbf{~ m m} / \mathbf{H g}$.

\begin{tabular}{ccc}
\hline Hour & $\begin{array}{c}\text { Differences in Mean } \\
\text { Pulmonary Artery } \\
\text { Systolic Pressure } \\
\text { (Unpaired t - test) }\end{array}$ & P-Value \\
\hline 1 & -0.95 & 0.34 \\
2 & -0.73 & 0.46 \\
3 & -0.68 & 0.49 \\
4 & -0.02 & 0.98 \\
5 & 0.58 & 0.56 \\
6 & 0.10 & 0.92 \\
7 & 0.07 & 0.94 \\
8 & 0.29 & 0.77 \\
\hline
\end{tabular}

Note. $*=$ Statistically significant

Table 4.21. Differences between Cases and Controls in Mean Mixed Venous Oxygen Saturation \%.

\begin{tabular}{ccc}
\hline Hour & $\begin{array}{c}\text { Differences in Mean } \\
\text { Mixed Venous } \\
\text { Oxygen Saturation } \\
\text { (Unpaired t-test) }\end{array}$ & P-Value \\
\hline 1 & -0.60 & 0.55 \\
2 & -0.83 & 0.40 \\
3 & 0.34 & 0.73 \\
4 & 0.18 & 0.85 \\
5 & -1.25 & 0.21 \\
6 & -1.53 & 0.12 \\
7 & -0.17 & 0.86 \\
8 & -1.73 & 0.08 \\
\hline
\end{tabular}

Note. $*=$ Statistically significant 


\begin{tabular}{|l|c|c|c|c|c|c|c|c|c|c|c|c|}
\hline $\begin{array}{c}\text { VARIABLE/ } \\
\text { TIME } \\
\text { (HOUR) }\end{array}$ & 1 & 2 & 3 & 4 & 5 & 6 & 7 & 8 & 9 & 10 & 11 & 12 \\
\hline $\mathrm{HR}$ & $\times$ & & & & & & & & & & & \\
\hline $\mathrm{MAP}$ & & & & & $\times$ & & & & & & & \\
\hline $\mathrm{CVP}$ & & & $\times$ & & & & $\times$ & $\times$ & $\times$ & $\times$ & & $\times$ \\
\hline $\mathrm{RR}$ & & $\times$ & $\times$ & $\times$ & $\times$ & $\times$ & $\times$ & $\times$ & $\times$ & $\times$ & $\times$ & $\times$ \\
\hline $\mathrm{SPO}$ & & & & $\times$ & $\times$ & $\times$ & & & & & & \\
\hline $\mathrm{CO}$ & $\times$ & $\times$ & $\times$ & $\times$ & $\times$ & $\times$ & $\times$ & $\times$ & $\times$ & & & $\times$ \\
\hline $\mathrm{CI}$ & $\times$ & & & & $\times$ & $\times$ & & $\times$ & & & & $\times$ \\
\hline $\mathrm{PAD}$ & $\times$ & $\times$ & $\times$ & $\times$ & $\times$ & $\times$ & & $\times$ & $\times$ & $\times$ & $\times$ & $\times$ \\
\hline $\mathrm{PASP}^{\mathrm{SVO}}$ & & & & & & & & & & & & \\
\hline
\end{tabular}

Figure 4.11. Cardiopulmonary Indicators' Significant Differences over Time.

Note. $\times$ - significant difference at $\mathrm{p}<0.05$

HR: heart rate

MAP: mean arterial pressure

CVP: central venous pressure

$\mathrm{RR}$ : respiratory rate

$\mathrm{SPO}_{2:}$ oxygen saturation

CO: cardiac output

CI: cardiac index

PAD: pulmonary diastolic pressure

PASP: pulmonary artery systolic pressure

$\mathrm{SVO}_{2}$ : mixed venous oxygen saturation 
Pearson correlation coefficient matrix of the CPI was generated through the REG procedure. The Pearson correlation coefficient matrix showing CPI that had significant effect on DVWR is presented in the Table 4.22. The correlation matrix revealed that there were significant correlations between CO, PAD, PASP, and MAP. MAP had significant correlations with CO and PASP. RR was significantly correlated with CO and PASP. CO had significant correlation with MAP and RR. PAD was significantly correlated with PASP. Although p-value showed significance in correlations between the above variables, all r-values were less than 0.5 . So, next R-square method was used to detect the multicollinearity.

Table 4.23 displays the regression model for all CPI variables. The R-square of the model was $=0.2454$, which indicated that the model was not over parameterized by the collinearity. By rule of thumb, R-square $\leq 0.25$ indicated that the model was free from multicollinearity (187). Further, multicollinearity was tested through the VIF and tolerance procedure.

Analysis of maximum likelihood estimates of CPI from the results of logistic regressions analysis are displayed in Table 4.24. The result indicated the significant predictors were MAP $(p=0.004), R R(p<0.0001)$, and PAD $(p=0.0004)$. It implies that MAP, RR, and PAD are statistically significant predictors of DVWR. Accordingly, the prediction equation for DVWR can be derived using CPI from Table 4.24 as shown below.

$$
\begin{gathered}
\text { Probability of DVWR }=\mathrm{P}(\mathrm{X}) / 1-\mathrm{P}(\mathrm{X})=\mathrm{e}^{\mathrm{x}} \\
\text { Where the } \mathrm{x}=-1.72-0.06(\mathrm{MAP})-0.19(\mathrm{RR})+0.23(\mathrm{PAD})
\end{gathered}
$$


Table 4.22. Correlation Matrix for Cardio Pulmonary Indicators.

\begin{tabular}{|c|c|c|c|c|c|}
\hline & $\begin{array}{c}\text { R Value } \\
\text { Mean MAP/ } \\
\text { P-Value } \\
\end{array}$ & $\begin{array}{c}\text { R Value } \\
\text { Mean RR/ } \\
\text { P-Value } \\
\end{array}$ & $\begin{array}{c}\text { R Value Mean } \\
\mathrm{CO} / \\
\text { P-Value } \\
\end{array}$ & $\begin{array}{c}\text { R Value } \\
\text { Mean PAD/ } \\
\text { P-Value }\end{array}$ & $\begin{array}{c}\text { R Value } \\
\text { Mean PASP/ } \\
\text { P-Value }\end{array}$ \\
\hline Mean & & -0.10068 & -0.20052 & 0.02464 & -0.1166 \\
\hline MAP & & $\mathrm{P}=0.0822$ & $\mathrm{P}=0.0005$ & $P=0.6713$ & $\mathrm{P}=0.0439$ \\
\hline Mean & & & 0.20855 & -0.05369 & 0.21322 \\
\hline RR & & & $\mathrm{P}=0.0003$ & $P=0.3549$ & $\mathrm{P}=0.0002$ \\
\hline Mean & & & & -0.07878 & 0.0856 \\
\hline $\mathrm{CO}$ & & & & $P=0.1743$ & $\mathrm{P}=0.1398$ \\
\hline Mean & & & & & 0.25175 \\
\hline PAD & & & & & $\mathrm{P}<0.0001$ \\
\hline
\end{tabular}

Note.

MAP: mean arterial pressure

RR: respiratory rate

CO: cardiac output

PAD: pulmonary diastolic pressure

PASP: pulmonary artery systolic pressure

Table 4.23. The Regression Model for Cardiopulmonary Indicators.

\begin{tabular}{cccccc}
\hline Source & DF & $\begin{array}{c}\text { Sum of } \\
\text { Square }\end{array}$ & $\begin{array}{c}\text { Mean } \\
\text { Square }\end{array}$ & F-Value & P-Value \\
\hline Model & 7 & 16.2517 & 2.32167 & 13.52 & $<0.0001$ \\
Error & 291 & 49.96904 & 0.17171 & & \\
Corrected & 298 & 66.22074 & & & \\
$\quad$ Total & & & & & \\
\hline
\end{tabular}

Note.

R-square: 0.2454

Adjusted R-square: 0.2273 
Table 4.24. Analysis of Maximum Likelihood Estimates of Cardiopulmonary Indicators.

\begin{tabular}{lrrrrr}
\hline \multirow{2}{*}{ Parameter } & DF & Estimate & Error & Chi-Square & $\begin{array}{c}\text { Wald } \\
\text { Pr }> \\
\text { Chi-Sq. }\end{array}$ \\
\hline Intercept & 1 & -1.7277 & 8.8035 & 0.0385 & 0.8444 \\
HR & 1 & 0.0055 & 0.0126 & 0.1921 & 0.6612 \\
MAP & 1 & -0.0606 & 0.0211 & 8.2830 & 0.0040 \\
CVP & 1 & 0.0116 & 0.0571 & 0.0410 & 0.8395 \\
RR & 1 & -0.1971 & 0.0489 & 16.2369 & $<.0001$ \\
SPO & 1 & 0.0546 & 0.0867 & 0.3969 & 0.5287 \\
CO & 1 & -0.3810 & 0.2278 & 2.7965 & 0.0945 \\
CI & 1 & 0.2682 & 0.5365 & 0.2499 & 0.6171 \\
PAD & 1 & 0.2319 & 0.0661 & 12.3236 & 0.0004 \\
PASP & 1 & -0.0537 & 0.0365 & 2.1688 & 0.1408 \\
$\mathrm{SVO}_{2}$ & 1 & 0.0384 & 0.0275 & 1.9467 & 0.1629 \\
\hline $\mathrm{NO}^{2}$ & 1 & & & &
\end{tabular}

Note.

HR: heart rate

MAP: mean arterial pressure

CVP: central venous pressure

$\mathrm{RR}$ : respiratory rate

$\mathrm{SPO}_{2:}$ oxygen saturation

CO: cardiac output

CI: cardiac index

PAD: pulmonary diastolic pressure

PASP: pulmonary artery systolic pressure

$\mathrm{SVO}_{2}$ : mixed venous oxygen saturation 


\section{Post Hoc Analysis}

Based on the descriptive and inferential statistics and the findings, the researcher performed a post hoc analysis in order to adjust for the extraneous variables associated with the research problem. The extraneous variables included in this study were demographic variables such as age, sex, and clinical variables such as BSA, number of grafts, and history of COPD, CHF, and renal failure.

Although age had shown no significant differences in distribution, age was included in the analysis as a covariate acknowledging the maturation effect of age on CPI. Sex was included as a covariate in the post hoc analysis considering the occurrence of an increased proportion of frequency distribution of female sex in the cases. Although BSA was not a study variable, it was included in the analysis as a covariate considering the effect of BSA in the CPI. Number of grafts was included as a covariate in the post hoc analysis because of its effect on the postoperative recovery and acuity of the patient.

The comparison of the demographic variables and clinical variables including age, sex, BSA, number of grafts, COPD, CHF, and renal failure between cases and controls was performed through a Chi-square test and PROC MIXED ANOVA. The final prediction model was built using logistic regression analysis by PROC LOGISTIC, including demographic and clinical variables as covariates with CPI.

Chi-square test: To find the differences in the age groups between cases and controls, a Chi-square test was performed. The analysis revealed that there was no significant difference in age groups between cases and controls. 
To find the differences in the sex groups between cases and controls, a Chi-square test was performed. The analysis revealed that there was a significant $(\mathrm{p}=0.01)$ difference in the distribution of sex between cases and controls. The relative risk estimated through odds ratio for sex was 0.52 , which is less than one. This result implies that sex is not a significant risk factor for developing DVWR after CABG surgery.

To find the differences in the prevalence of comorbidities between cases and controls, a Chi-square test was performed. The analysis revealed that there was no significant difference in the prevalence of renal failure between cases and controls. However, the prevalence of COPD showed a significant $(\mathrm{p}<0.0001)$ difference between cases and controls. Odds ratio of COPD was 5.11, which implies that the odds in favor of DVWR for patients with COPD are 5.11 times higher than for patients without COPD. In addition, the prevalence of CHF showed a significant $(p=0.0003)$ difference between cases and controls. Relative risk was estimated through odds ratio of CHF was 6.75, which implies that the odds in favor of DVWR for patients with CHF were 6.75 times higher than for patients without CHF.

To describe the differences between cases and controls in the number of grafts, a Chi-square test was preformed, which revealed that there was no significant difference in the number of grafts between cases and controls.

A comparison was performed between cases and controls to detect the differences in the means of BSA through an unpaired t-test. The analysis revealed no significant difference in mean BSA.

In summary, results of the Chi-square test revealed no significant difference in the demographic variables such as BSA, number of grafts, and renal failure among cases and 
controls. However, there were significant differences in the prevalence of COPD ( $p<$ 0.0001) and CHF ( $p=0.0003)$ among cases and controls. In addition, there was a significant difference $(p=0.01)$ in sex when comparing cases and controls. Further, Chisquare for age groups showed a significant $(\mathrm{p}=0.05)$ difference between cases and controls.

ANOVA procedure: Next, the effect of the demographic, clinical, and comorbidity variables on CPI was tested through an ANOVA for a repeated-measures model-based design using the PROC MIXED procedure of SAS including the above variables as covariates in the analysis. The covariates in this analysis included age, sex, BSA, number of grafts, COPD, CHF, and renal failure.

The first eight repeated measure means were generated for CPI values in eight hourly time points for cases and controls. The comparison of group means was performed using ANOVA for repeated measures through mixed procedure (PROC MIXED). This procedure was selected because it is specifically designed for mixed effects models. In addition, PROC MIXED procedure was selected to accommodate the following considerations in analysis.

The first consideration was that this research data contained a considerable amount of missing data. To address this issue, the PROC MIXED REPEATED MEASURE ANOVA was selected, since it handles missing data and applies multiple comparison procedures between and within subjects' factors. Unlike other programs, the PROC MIXED handles all of the technical details itself through a likelihood-based estimation method. Further, it knows the proper way to construct its test statistics to 
account for the fixed and random nature of the study factors. In addition, it provides many important, unique features such as provision for a larger class of covariance structures and a better mechanism of handling missing data through estimates of covariance by restricted maximum likelihood computations and comparisons.

The next consideration was to control for the effects of covariates on the outcome variable. As this research study involved multiple covariates, controlling for their effect was mandatory while addressing this research question. When assessing the nature of this study data, CPI may be influenced by the effect of various covariates such as age, sex, COPD, CHF, renal failure, BSA, and number of grafts. With repeated measures analysis of variance, measurements made on the same subject are likely to be more similar than measurements made on different individuals - that is, repeated measures are correlated. For an analysis to be valid, the covariance among repeated measures must be modeled properly. The PROC MIXED ANOVA for repeated measures computes LSMEANS which are averaged across repeated measures and whose standard errors reflect the appropriate covariance structure. The covariance structure used in this study was compound symmetry (CS), which implies that the correlations between all pairs of measures are the same. This process was accomplished through the PROC MIXED repeated measures ANOVA program, in order to have control the effects of repeated measures arising from different sets of conditions, such as the response to different conditions.

The methods implemented in the PROC MIXED were based on an assumption of normally distributed residuals and independent, constant variances across groups. The PROC MIXED uses a restricted maximum likelihood-based estimation routine (REML) 
based on normal distribution theory and therefore does not compute or display sums of squares, as observed with PROC GLM.

A null and alternative hypothesis was formulated as $\mathrm{H}_{0}$ : All means are equal vs. at least one means is different with alpha of 0.05 . The restricted maximum likelihood-based estimation routine (REML) uses a weighted average of the individual sample variances. The results are presented below as ANOVA tables for all the CPI.

The ANOVA table (Table 4.25) showed no significant differences in the means of heart rate between DVWR (cases) and NVWR (controls) groups. However, there was a significant $(p=0.0005)$ difference in the effect of time on the heart rate means between cases and controls. Age had a significant $(p=0.008)$ effect on HR. In contrast, covariates such as, sex, BSA, CHF, COPD, renal failure, and number of grafts did not have a significant effect on the heart rate means of cases and controls.

The ANOVA table (Table 4.26) showed no significant differences in the group means of MAP between DVWR (cases) and NVWR (controls) groups. However, there were significant differences in the effect of age $(p=0.0028)$ and $\operatorname{sex}(p=0.0012)$ on the group means of MAP between cases and controls. Covariates such as BSA, CHF, COPD, renal failure, and number of grafts did not have a significant effect on the group means of MAP cases and controls.

The ANOVA table (Table 4.27) showed no significant differences in the group means of CVP between DVWR (cases) and NVWR (control) groups. However, there was a significant $(p<0.0001)$ difference in effect of time on group means of CVP between 
Table 4.25. ANOVA Table for Heart Rate (HR) Beat per Minute Post Hoc.

\begin{tabular}{ccccc}
\hline Effect & $\begin{array}{c}\text { Numerator } \\
\text { DF }\end{array}$ & $\begin{array}{c}\text { Denominator } \\
\text { DF }\end{array}$ & F-Value & $\operatorname{Pr}>$ F \\
\hline Case & 1 & 290 & 1.4 & 0.2371 \\
Hour & 7 & 2079 & 3.88 & 0.0003 \\
Case*Hour & 7 & 2079 & 3.88 & 0.0003 \\
Age & 1 & 290 & 7.05 & 0.0083 \\
Sex & 1 & 290 & 0.86 & 0.3538 \\
BSA & 1 & 290 & 0.6 & 0.4404 \\
CHF & 1 & 290 & 0.13 & 0.7185 \\
COPD & 1 & 290 & 0.88 & 0.3499 \\
Renal & 1 & 290 & 0.04 & 0.8352 \\
Grafts & 1 & 290 & 1.57 & 0.2107 \\
\hline
\end{tabular}

Note. Case*Hour means interaction of cases and controls with extubation time.

BSA: body surface area

CHF: congestive heart failure

COPD: chronic obstructive pulmonary disease

Table 4.26. ANOVA Table for Mean Arterial Pressure (MAP) mm/Hg Post Hoc.

\begin{tabular}{ccccc}
\hline Effect & $\begin{array}{c}\text { Numerator } \\
\text { DF }\end{array}$ & $\begin{array}{c}\text { Denominator } \\
\text { DF }\end{array}$ & F-Value & $\operatorname{Pr}>\mathrm{F}$ \\
\hline Case & 1 & 290 & 2.72 & 0.1001 \\
Hour & 7 & 2079 & 16.45 & $<.0001$ \\
Case*Hour & 7 & 2079 & 1.61 & 0.1273 \\
Age & 1 & 290 & 9.1 & 0.0028 \\
Sex & 1 & 290 & 10.65 & 0.0012 \\
BSA & 1 & 290 & 2.74 & 0.0988 \\
CHF & 1 & 290 & 0.28 & 0.5978 \\
COPD & 1 & 290 & 1.94 & 0.1648 \\
Renal & 1 & 290 & 0.42 & 0.5186 \\
Grafts & 1 & 290 & 0.14 & 0.712 \\
\hline
\end{tabular}

Note. Case*Hour means interaction of cases and controls with extubation time.

BSA: body surface area

CHF: congestive heart failure

COPD: chronic obstructive pulmonary disease 
Table 4.27. ANOVA Table for Central Venous Pressure (CVP) cm/ $\mathrm{H}_{2} \mathrm{O}$ Post Hoc.

\begin{tabular}{ccccl}
\hline Effect & $\begin{array}{c}\text { Numerator } \\
\text { DF }\end{array}$ & $\begin{array}{c}\text { Denominator } \\
\text { DF }\end{array}$ & $\begin{array}{c}\text { F- } \\
\text { Value }\end{array}$ & Pr F \\
\hline Case & 1 & 290 & 2.25 & 0.1346 \\
Hour & 7 & 2079 & 4.67 & $<.0001$ \\
Case*Hour & 7 & 2079 & 4.52 & $<.0001$ \\
Age & 1 & 290 & 0.85 & 0.3574 \\
Sex & 1 & 290 & 0.02 & 0.8851 \\
BSA & 1 & 290 & 4.09 & 0.044 \\
CHF & 1 & 290 & 0.07 & 0.7903 \\
COPD & 1 & 290 & 0.1 & 0.7573 \\
Renal & 1 & 290 & 0.18 & 0.6697 \\
Grafts & 1 & 290 & 3.72 & 0.0546 \\
\hline
\end{tabular}

Note. Case*Hour means interaction of cases and controls with extubation time.

BSA: body surface area

$\mathrm{CHF}$ : congestive heart failure

COPD: chronic obstructive pulmonary disease 
cases and controls. Covariates such as age, sex, CHF, COPD, renal failure, and number of grafts did not have a significant effect on heart rate means of cases and controls. There was also a significant $(p=0.044)$ effect of BSA on the group means of CVP.

The ANOVA table (Table 4.28) showed significant $(\mathrm{p}<0.001)$ differences in the group means of RR between DVWR (cases) and NVWR (controls). In addition, there were significant $(p<0.0001)$ difference in the effect of time on the group means of RR. Further there were significant $(\mathrm{p}=0.0086)$ differences in the effect of number of grafts and BSA $(p=0.0028)$ on the group means of RR. Covariates such as age, sex, CHF, COPD, and renal failure did not have a significant effect on the group means of respiratory rate.

The ANOVA table (Table 4.29) showed a significant $(\mathrm{p}=0.054)$ difference in the group means of $\mathrm{SPO}_{2}$ between DVWR (cases) and NVWR (controls). In addition, there were significant differences in the effect covariates such as age $(p=0.042)$, BSA $(p=$ $0.0034)$, renal failure $(p=0.019)$, and number of grafts $(p=0.057)$ on the group means of $\mathrm{SPO}_{2}$. However, covariates such as sex, $\mathrm{CHF}$, and COPD, did not have a significant effect on the group mean $\mathrm{SPO}_{2}$ of cases and controls.

The ANOVA table (Table 4.30) showed that there are significant $(\mathrm{p}=0.03)$ differences in the group means of CO between DVWR (cases) and NVWR (control) groups. In addition, covariates such as age $(p=0.015)$, sex $(p<0.0001)$, and BSA $(p=0.0001)$ had a significant effect on the group means of CO. Other covariates such as CHF, COPD, renal failure, and number of grafts did not have a significant effect on the group means of $\mathrm{CO}$. 
Table 4.28. ANOVA Table for Respiratory Rate (RR) per Minute Post Hoc.

\begin{tabular}{ccccc}
\hline Effect & $\begin{array}{c}\text { Numerator } \\
\text { DF }\end{array}$ & $\begin{array}{c}\text { Denominator } \\
\text { DF }\end{array}$ & F-Value & Pr $>$ F \\
\hline Case & 1 & 290 & 32.51 & $<.0001$ \\
Hour & 7 & 2079 & 50.99 & $<.0001$ \\
Case*Hour & 7 & 2079 & 5.11 & $<.0001$ \\
Age & 1 & 290 & 0.27 & 0.6065 \\
Sex & 1 & 290 & 0.01 & 0.9202 \\
BSA & 1 & 290 & 9.07 & 0.0028 \\
CHF & 1 & 290 & 0.1 & 0.9673 \\
COPD & 1 & 290 & 0.46 & 0.4997 \\
Renal & 1 & 290 & 0.15 & 0.7033 \\
Grafts & 1 & 290 & 7 & 0.0086 \\
\hline
\end{tabular}

Note. Case*Hour means interaction of cases and controls with extubation time. BSA: body surface area

CHF: congestive heart failure

COPD: chronic obstructive pulmonary disease

Table 4.29. ANOVA Table for Oxygen Saturation $\left(\mathrm{SPO}_{2}\right) \%$ Post Hoc.

\begin{tabular}{ccccc}
\hline Effect & $\begin{array}{c}\text { Numerator } \\
\text { CF }\end{array}$ & $\begin{array}{c}\text { Denominator } \\
\text { DF }\end{array}$ & F-Value & $\operatorname{Pr}>$ F \\
\hline Hour & 1 & 290 & 3.74 & 0.0542 \\
Case*Hour & 7 & 2079 & 3.96 & 0.0003 \\
Age & 1 & 2079 & 0.59 & 0.7625 \\
Sex & 1 & 290 & 4.16 & 0.0423 \\
BSA & 1 & 290 & 0.01 & 0.9238 \\
CHF & 1 & 290 & 8.73 & 0.0034 \\
COPD & 1 & 290 & 0.58 & 0.4475 \\
Renal & 1 & 290 & 1.23 & 0.2682 \\
Grafts & 1 & 290 & 5.51 & 0.0196 \\
\hline
\end{tabular}

Note. Case*Hour means interaction of cases and controls with extubation time.

BSA: body surface area

CHF: congestive heart failure

COPD: chronic obstructive pulmonary disease 
Table 4.30. ANOVA Table for Cardiac Output (CO) Liter per Minute Post Hoc.

\begin{tabular}{ccccc}
\hline Effect & $\begin{array}{c}\text { Numerator } \\
\text { DF }\end{array}$ & $\begin{array}{c}\text { Denominator } \\
\text { DF }\end{array}$ & F-Value & $\operatorname{Pr}>\mathrm{F}$ \\
\hline Case & 1 & 290 & 4.71 & 0.0308 \\
Hour & 7 & 2079 & 11.35 & $<.0001$ \\
Case*Hour & 7 & 2079 & 1.23 & 0.2804 \\
Age & 1 & 290 & 5.94 & 0.0154 \\
Sex & 1 & 290 & 26.18 & $<.0001$ \\
BSA & 1 & 290 & 42.34 & $<.0001$ \\
CHF & 1 & 290 & 2.2 & 0.1395 \\
COPD & 1 & 290 & 0.94 & 0.3327 \\
Renal & 1 & 290 & 0.59 & 0.4436 \\
Grafts & 1 & 290 & 0.11 & 0.7385 \\
\hline
\end{tabular}

Note. Case*Hour means interaction of cases and controls with extubation time.

BSA: body surface area

$\mathrm{CHF}$ : congestive heart failure

COPD: chronic obstructive pulmonary disease 
The ANOVA table (Table 4.31) showed significant $(\mathrm{p}=0.03)$ differences in the group means of CI between DVWR (cases) and NVWR (control) group. In addition, covariates such as age $(p=0.016)$, sex $(p<0.0001)$, and BSA $(p=0.0001)$ had a significant effect on the group means of CI. Other covariates such as CHF, COPD, renal failure, and number of grafts did not have a significant effect on the group means of CI.

The ANOVA table (Table 4.32) showed significant $(p=0.0012)$ differences in the group means of PAD between DVWR (cases) and NVWR (control) groups. In addition, there was also a significant effect $(p=0.011)$ of time on the group means of PAD. Age also had a significant effect on PAD $(p=0.04)$. In contrast, covariates such as sex, BSA, CHF, COPD, renal failure, and number of grafts did not have significant effect on the group means of PAD in cases and controls.

The ANOVA table (Table 4.33) showed no significant differences in the group means of PASP between DVWR (cases) and NVWR (control) groups. However, there were significant effects of covariates such as BSA $(p=0.0059)$, CHF $(p=0.0034)$, and number of grafts ( $p$ 0.05) on the group means of PASP. Covariates such as age, sex, COPD, and renal failure did not have a significant effect on the group means of PASP in cases and controls.

The ANOVA table (Table 4.34) showed no significant difference in the group means of $\mathrm{SVO}_{2}$ between DVWR (cases) and NVWR (controls). In addition, there was no significant effect of time on the group means of $\mathrm{SVO}_{2}$ in cases and controls. Covariates such as age, sex, BSA, CHF, COPD, renal failure, and number of grafts did not have a significant effect on the group means $\mathrm{SVO}_{2}$ in cases and controls. 
Table 4.31. ANOVA Table for Cardiac Index (CI) Liter per Minute Post Hoc.

\begin{tabular}{ccccl}
\hline Effect & $\begin{array}{c}\text { Numerator } \\
\text { DF }\end{array}$ & $\begin{array}{c}\text { Denominator } \\
\text { DF }\end{array}$ & F-Value & Pr $>$ F \\
\hline Case & 1 & 290 & 4.29 & 0.0391 \\
Hour & 7 & 2079 & 13.05 & $<.0001$ \\
Case*Hour & 7 & 2079 & 1.1 & 0.3619 \\
Age & 1 & 290 & 5.87 & 0.016 \\
Sex & 1 & 290 & 17.53 & $<.0001$ \\
BSA & 1 & 290 & 20.39 & $<.0001$ \\
CHF & 1 & 290 & 2.53 & 0.1129 \\
COPD & 1 & 290 & 0.78 & 0.3765 \\
Renal & 1 & 290 & 0.86 & 0.3559 \\
Grafts & 1 & 290 & 1.28 & 0.2589 \\
\hline
\end{tabular}

Note. Case*Hour means interaction of cases and controls with extubation time.

BSA: body surface area

CHF: congestive heart failure

COPD: chronic obstructive pulmonary disease

Table 4.32. ANOVA Table for Pulmonary Artery Diastolic Pressure (PAD) mm/Hg Post Hoc.

\begin{tabular}{ccccl}
\hline Effect & $\begin{array}{c}\text { Numerator } \\
\text { DF }\end{array}$ & $\begin{array}{c}\text { Denominator } \\
\text { DF }\end{array}$ & F-Value & Pr $>$ F \\
\hline Case & 1 & 290 & 10.66 & 0.0012 \\
Hour & 7 & 2079 & 1.63 & 0.1225 \\
Case*Hour & 7 & 2079 & 2.6 & 0.0113 \\
Age & 1 & 290 & 3.99 & 0.0467 \\
Sex & 1 & 290 & 0.04 & 0.8392 \\
BSA & 1 & 290 & 2.57 & 0.11 \\
CHF & 1 & 290 & 1 & 0.3184 \\
COPD & 1 & 290 & 0.19 & 0.666 \\
Renal & 1 & 290 & 0.05 & 0.8275 \\
Grafts & 1 & 290 & 0.01 & 0.9243 \\
\hline
\end{tabular}

Note. Case*Hour means interaction of cases and controls with extubation time.

BSA: body surface area

CHF: congestive heart failure

COPD: chronic obstructive pulmonary disease 
Table 4.33. ANOVA Table for Pulmonary Artery Systolic Pressure (PASP) mm/Hg Post Hoc.

\begin{tabular}{cccll}
\hline Effect & $\begin{array}{c}\text { Numerator } \\
\text { DF }\end{array}$ & $\begin{array}{c}\text { Denominator } \\
\text { DF }\end{array}$ & F-Value & $\operatorname{Pr}>\mathrm{F}$ \\
\hline Case & 1 & 290 & 0.03 & 0.8606 \\
Hour & 7 & 2079 & 2.75 & 0.0076 \\
Case*Hour & 7 & 2079 & 0.69 & 0.6811 \\
Age & 1 & 290 & 0 & 0.9673 \\
Sex & 1 & 290 & 1.46 & 0.2272 \\
BSA & 1 & 290 & 7.7 & 0.0059 \\
CHF & 1 & 290 & 8.71 & 0.0034 \\
COPD & 1 & 290 & 1.59 & 0.2087 \\
Renal & 1 & 290 & 0.28 & 0.5978 \\
Grafts & 1 & 290 & 3.86 & 0.0503 \\
\hline
\end{tabular}

Note. Case*Hour means interaction of cases and controls with extubation time.

BSA: body surface area

$\mathrm{CHF}$ : congestive heart failure

COPD: chronic obstructive pulmonary disease

Table 4.34. ANOVA Table for Mixed Venous Oxygen Saturation $\left(\mathrm{SVO}_{2}\right) \%$ Post Hoc.

\begin{tabular}{ccccc}
\hline Effect & $\begin{array}{c}\text { Numerator } \\
\text { DF }\end{array}$ & $\begin{array}{c}\text { Denominator } \\
\text { DF }\end{array}$ & F-Value & Pr $>$ F \\
\hline Case & 1 & 290 & 1.26 & 0.2626 \\
Hour & 7 & 2079 & 1.52 & 0.1564 \\
Case*Hour & 7 & 2079 & 1.15 & 0.3259 \\
Age & 1 & 290 & 1.84 & 0.1758 \\
Sex & 1 & 290 & 0.13 & 0.7165 \\
BSA & 1 & 290 & 3.34 & 0.0686 \\
CHF & 1 & 290 & 2.03 & 0.1556 \\
COPD & 1 & 290 & 1.94 & 0.1649 \\
Renal & 1 & 290 & 0.06 & 0.8112 \\
Grafts & 1 & 290 & 1.87 & 0.1723 \\
\hline
\end{tabular}

Note. Case*Hour means interaction of cases and controls with extubation time.

BSA: body surface area

CHF: congestive heart failure

COPD: chronic obstructive pulmonary disease 
Multicollinearity test: Tolerance and variance inflation factors (VIF) were generated to diagnose the multicollinearity and are displayed in Table 4.35. Tolerance and variance inflation factors (VIF) are two reciprocal parameters that can be used to diagnose multicollinearity. Tolerance $=1-\mathrm{R}$-square, where R-square is the coefficient of determination for regression of that variable on all remaining variables. Low tolerance values indicate high multivariate correlations (187). This model contained variables that had tolerances ranging from 0.844 to 0.975 , which indicated that there was no significant multivariate correlation in this model. Further, the values of VIF exceeding 10 in larger sample size and 2.5 in smaller sample size were regarded as indicating multicollinearity (187). This model did not contain any variable with VIF more than 2.5 , which indicated that multicollinearity was not the problem.

Logistic regression procedure: This model included major confounding variables such as age, sex, BSA, number of grafts, COPD, CHF, and renal diseases as antecedence. Further, the researcher made a linearity assumption for performing a logistic regression procedure. In order to assume linearity, the relationships between predictors and the outcome variable should be linear; however, this study contained categorical outcome variables that did not meet the linearity assumption. In order to achieve linearity, the data was transformed by natural logarithm. The natural logarithm of the odds is called the logit. The $\operatorname{logit}=\ln \mathrm{P}(\mathrm{x}) / 1-\mathrm{P}(\mathrm{x})$. Logistic regression procedure was performed to build a prediction model. 
Table 4.35. Parameter Estimates of Regression Model for Dysfunctional Ventilator Weaning Response with Antecedence Post Hoc.

\begin{tabular}{lcccccc}
\hline Variable & DF & $\begin{array}{l}\text { Parameter } \\
\text { Estimate }\end{array}$ & Std. Error & T - Value & Tolerance & VIF \\
\hline Intercept & 1 & 1.88446 & 0.382 & 4.93 & & 0 \\
COPD & 1 & 0.31798 & 0.079 & 3.99 & 0.975 & 1.025 \\
CHF & 1 & 0.3034 & 0.109 & 2.77 & 0.943 & 1.059 \\
Mean MAP & 1 & -0.009 & 0.003 & -2.45 & 0.935 & 1.069 \\
Mean RR & 1 & -0.04008 & 0.007 & -5.46 & 0.896 & 1.115 \\
Mean CO & 1 & -0.0635 & 0.025 & -2.45 & 0.901 & 1.109 \\
Mean PAD & 1 & 0.00755 & 0.002 & 2.65 & 0.916 & 1.091 \\
Mean PASP & 1 & 0.00002361 & 0.004 & 0.01 & 0.844 & 1.183 \\
\hline
\end{tabular}

Note. Antecedence indicates significant predictors of dysfunctional ventilator weaning response (DVWR).

COPD: chronic obstructive pulmonary disease

$\mathrm{CHF}$ : congestive heart failure

MAP: mean arterial pressure

RR: mean respiratory rate

CO: mean cardiac output

PAD: mean pulmonary diastolic pressure

PASP: mean pulmonary artery systolic pressure 
Stepwise procedure. A stepwise procedure with the combination of forward selection and backward elimination was performed. The variables were selected into the multiple regression models by using a stepwise procedure. The significant level of entry is $p$-value $<0.05$, and the significant level of elimination is $p$-value $>0.05$. All the explanatory variables including CPI, demographic, clinical and comorbidities were included in the analysis. From the summary of the stepwise procedure, analysis of maximum likelihood estimates were generated and presented in Table 4.36.

Maximum likelihood analysis. The analysis of maximum likelihood identifies independent variables that had a significant effect on the dependent variable DVWR. Likelihood is a probability — specifically the probability that the observed values of the dependent variable may be predicted from the observed values of the independents. The log likelihood varies from minus to infinity. Log likelihood is calculated through iteration, using maximum likelihood estimation. It is the basis for tests of a logistic model. A maximum likelihood estimation reflects how likely it is (the odds) that the observed values of the DVWR may be predicted from the observed values of the independent variables.

An analysis of maximum likelihood estimates table consisted of all the variables that had a significant effect on the dependent variable DVWR $(\mathrm{P}(\mathrm{Y}=1))$, which included mean COPD $(p=0.003)$, mean CHF $(p=0.0431)$, mean MAP $(p=0.0055)$, mean RR $(\mathrm{p}<0.0001)$, mean CO $(\mathrm{p}=0.0382)$, mean PAD $(\mathrm{p}<0.0001)$, and mean PASP $(p=0.0061)$ 
Table 4.36. Analysis of Maximum Likelihood Estimates of Antecedence Post Hoc.

\begin{tabular}{lccccc}
\hline Parameter & DF & Estimate & $\begin{array}{c}\text { Standard } \\
\text { Error }\end{array}$ & $\begin{array}{c}\text { Wald Chi- } \\
\text { square }\end{array}$ & P-Value \\
\hline Intercept & 1 & 8.9452 & 2.5166 & 12.6343 & 0.0004 \\
COPD & 1 & 1.6985 & 0.4655 & 13.3108 & 0.0003 \\
CHF & 1 & 1.3687 & 0.6766 & 4.0928 & 0.0431 \\
Mean MAP & 1 & -0.065 & 0.0234 & 7.6919 & 0.0055 \\
Mean RR & 1 & -0.2183 & 0.0486 & 20.191 & $<0.0001$ \\
Mean CO & 1 & -0.3322 & 0.1603 & 4.2943 & 0.0382 \\
Mean PAD & 1 & 0.2584 & 0.0628 & 16.9208 & $<0.0001$ \\
Mean PASP & 1 & -0.1116 & 0.0407 & 7.5068 & 0.0061 \\
\hline
\end{tabular}

Note. Antecedence indicates significant predictors of dysfunctional ventilator weaning response (DVWR).

COPD: chronic obstructive pulmonary disease

$\mathrm{CHF}$ : congestive heart failure

MAP: mean arterial pressure

$\mathrm{RR}$ : mean respiratory rate

$\mathrm{CO}$ : mean cardiac output

PAD: mean pulmonary diastolic pressure

PASP: mean pulmonary artery systolic pressure 
The parameter estimates were negative for the variables, including mean MAP, mean RR, mean CO, and mean PASP, which implies that the probability of DVWR $(\mathrm{P}(\mathrm{Y}=1))$ declines when the variables' mean increases, whereas the probability of DVWR (P $(\mathrm{Y}=1))$ increases with an increase in mean COPD, mean CHF, and mean PAD. The predictive equation was calculated with the following logistic regression parameters:

The logit $($ DVWR $)=\ln P(x) / 1-P(x)=e^{x}$ where $\mathrm{x}=8.94+1.69(C O P D)+1.36(C H F)-$ 0.06 (Mean MAP) - $0.21($ Mean RR $)-0.33($ Mean CO $)+0.25($ Mean PAD $)-0.11$ (Mean PASP).

Likelihood confidence and odds ratio. Next, to quantify the effect of the significant independent variables on the dependent variable DVWR, a likelihood confidence interval and odds ratio was estimated. A profile of likelihood confidence intervals for adjusted odds ratio was estimated and is presented in Table 4.36. The odds ratio contains the exponentials of the logistic regression coefficients of the significant independent variables comparing DVWR (cases) with NVWR (controls).

Table 4.37 displays the significant independent variables that had a significant effect on DVWR. The results displayed included unit estimates of odds ratio and $95 \%$ confidence limits. The odds in favor of DVWR for patients with COPD were 5.466 times higher than for patients without COPD, holding all other variables constant. The odds in favor of DVWR for patients with CHF were 3.930 times higher than the patients without CHF, holding all other variables constant. 
Table 4.37. Profile of Likelihood Confidence Interval for Adjusted Odds Ratios of Antecedence Post Hoc.

\begin{tabular}{lrcc}
\hline \multicolumn{1}{c}{ Effect } & Unit & $\begin{array}{c}\text { Estimate - } \\
\text { Odds }\end{array}$ & 95\% Confidence Limit \\
\hline COPD & 1 & 5.466 & $2.246-14.131$ \\
CHF & 1 & 3.93 & $1.118-16.747$ \\
Mean MAP & -10 & 1.915 & $1.219-3.063$ \\
Mean RR & -5 & 2.978 & $1.878-4.884$ \\
Mean CO & -2 & 1.943 & $1.050-3.717$ \\
Mean PAD & 5 & 3.64 & $2.009-6.913$ \\
Mean PASP & -10 & 3.053 & $1.406-6.981$ \\
\hline
\end{tabular}

Note. Antecedence indicates significant predictors of dysfunctional ventilator weaning response (DVWR).

COPD: chronic obstructive pulmonary disease

$\mathrm{CHF}$ : congestive heart failure

MAP: mean arterial pressure

$\mathrm{RR}$ : mean respiratory rate

$\mathrm{CO}$ : mean cardiac output

PAD: mean pulmonary diastolic pressure

PASP: mean pulmonary artery systolic pressure 
The odds in favor of DVWR for patients with a decrease $10 \mathrm{~mm} / \mathrm{Hg}$ mean MAP were 1.915 times of the probability of NVWR, holding all other variables constant. This finding implies that hypotension increases the risk of developing DVWR after CABG surgery.

The odds in favor of DVWR for patients with a decrease of 5 points of mean RR were 2.978 times the probability of NVWR, holding all other variables constant. This finding implies that patients with a lower RR are at risk of developing DVWR after CABG surgery.

The odds in favor of DVWR for patients with a decrease in mean CO by 2 points were 1.943 times of probability of NVWR, holding all other variables constant. This finding implies that patients with a low CO are at the risk of developing DVWR after CABG surgery.

The odds in favor of DVWR for patients with an increase in mean PAD by $5 \mathrm{~mm} / \mathrm{Hg}$ were 3.640 times of the probability of NVWR, holding all other variables constant. This finding implies that the patients with a high PAD pressure are at the risk of developing DVWR after CABG surgery.

The odds in favor of DVWR for patients with decrease in mean PASP by $10 \mathrm{~mm} / \mathrm{Hg}$ were 3.053 times of the probability of NVWR, holding all other variables constant. This finding implies that the patients with low PASP are at the risk of developing DVWR after CABG surgery.

Goodness-of-fit test. Next, the goodness-of-fit test was done to test the model by the Hosmer and Lemshow method. The LACKFIT option was used to print the Hosmer 
and Lemshow goodness-of-fit test. The Chi-square was 9.7845, with df 8 at significance level of $p=0.2805$, which is higher than $p<0.05$, which shows that there was no lack of fitness in this model.

Prediction accuracy analysis. Further, the model was tested for prediction accuracy through a classification table. The accuracy of the classification was measured by its sensitivity, which is the ability to predict an event correctly, and specificity, which is the ability to predict non-event. The sensitivity of the model was $77.8 \%$, and specificity was $74.5 \%$ at a 0.3 probability level.

\section{Summary of Analysis}

Data analysis was guided by the preplanned research questions. The results were organized and displayed under the respective research questions. This researcher utilized descriptive and inferential statistics to derive answers to the research questions. The analysis included preplanned and post hoc comparisons of study variables and covariates. Statistical analysis was performed through SAS programs, including PROC UNIVARIATE, PROC FREQ, PROC GLM, PROC REG, PROC MIXED REPEATED MEASURE ANOVA, and PROC LOGISTIC. The total sample size was 300; however, only 299 subjects were included in the data analysis, which contained 99 cases and 200 controls.

The results of this case control study with time series design revealed that selected covariates had an effect on the characteristics of CPI after CABG surgery. Covariates such as age, sex, BSA, time, number of grafts, CHF, and renal failure had significant 
effect on the characteristics of certain CPI such as MAP, CVP, RR, $\mathrm{SPO}_{2}, \mathrm{CO}, \mathrm{CI}, \mathrm{PAD}$, and PASP after CABG surgery. Age and sex had a significant effect on the mean MAP. Time had a significant $(\mathrm{p}<0.0001)$ effect on the group means of CVP in cases and controls. Time also had a significant $(\mathrm{p}<0.0001)$ effect on the group means of RR. Number of grafts had a significant $(p=0.0086)$ effect on the group means of RR. This study found that there was a significant effect of covariates such as age $(p=0.042)$, BSA $(p=0.0034)$, renal failure $(p=0.019)$, and number of grafts $(p=0.057)$ on the group means of $\mathrm{SPO}_{2}$. Covariates such as age $(\mathrm{p}=0.015)$, sex $(\mathrm{p}<0.0001)$, and $\mathrm{BSA}(\mathrm{p}=$ 0.0001) had significant effect on the group means of CO. Furthermore, the covariates such as age $(p=0.016)$, sex $(p<0.0001)$, and BSA $(p=0.0001)$ had significant effect on the group means of CI. The mean PAD was found to have been affected by time significantly $(\mathrm{p}=0.011)$. The mean PASP was significantly affected by covariates such as BSA $(p=0.0059), \operatorname{CHF}(p=0.0034)$, and number of grafts $(p=0.05)$.

The research study findings revealed that there were significant differences between cases and controls in the characteristics of certain $\mathrm{CPI}$ such as $\mathrm{RR}, \mathrm{SPO}_{2}, \mathrm{CO}, \mathrm{CI}$, and PAD after CABG surgery. There was a significant $(\mathrm{p}<0.001)$ difference in the group means of RR between DVWR (cases) and NVWR (controls). There was also a significant $(\mathrm{p}=0.054)$ difference in the group means of $\mathrm{SPO}_{2}$ between DVWR (cases) and NVWR (controls) noted. In addition, there was a significant $(\mathrm{p}=0.03)$ difference in the group means of CO between DVWR (cases) and NVWR (controls) groups. Further, there was a significant $(\mathrm{p}=0.03)$ difference in the group means of CI between DVWR (cases) and NVWR (control) groups. There was also a significant $(p=0.0012)$ difference in the group means of PAD between DVWR (cases) and NVWR (controls). 
These research study findings also revealed that there were no significant differences between cases and controls in the characteristics of certain CPI such as HR, MAP, CVP, PASP, and $\mathrm{SPO}_{2}$ after CABG surgery. In addition, the findings revealed that covariate COPD did not have any effect on the characteristics of CPI among cases and controls after CABG surgery.

The findings also revealed that antecedence such as COPD, CHF, MAP, RR, CO, PAD, and PASP had a significant effect on DVWR. The findings further revealed that the odds in favor of DVWR for patients with COPD are 5.466 times higher than for patients without COPD, holding all other variables constant. The odds in favor of DVWR for patients with CHF were 3.930 times higher than the patients without CHF, holding all other variables constant. The odds in favor of DVWR for patients with a decrease of $10 \mathrm{~mm} / \mathrm{Hg}$ in the mean MAP was 1.915 times the probability of NVWR, holding all other variables constant. This finding implies that hypotension increases the risk of developing DVWR after CABG surgery. The odds in favor of DVWR for patients with a decrease of 5 points in mean RR was 2.978 times the probability of NVWR, holding all other variables constant. This finding implies that patients with a lower $R R$ are at risk of developing DVWR after CABG surgery. The odds in favor of DVWR for patients with decrease in mean CO by 2 points was 1.943 times the probability of NVWR, holding all other variables constant. This finding implies that patients with low $\mathrm{CO}$ are at the risk of developing DVWR after CABG surgery. The odds in favor of DVWR for patients with an increase in mean PAD by $5 \mathrm{~mm} / \mathrm{hg}$ was 3.640 times the probability of NVWR, holding all other variables constant. This finding implies that the patients with high PAD pressure are at the risk of developing DVWR after CABG surgery. The odds in favor of DVWR 
for patients with a decrease in mean PASP by $10 \mathrm{~mm} / \mathrm{Hg}$ were 3.053 times the probability of NVWR, holding all other variables constant. This finding implies that the patients with low PASP are at risk of developing DVWR after CABG surgery.

In summary, the antecedence-including COPD, CHF, MAP, RR, CO, PAD, and PASP — had a significant effect on DVWR after CABG surgery. These antecedence were found effective in building a prediction model for DVWR after CABG surgery. 


\section{CHAPTER 5: CONCLUSION}

\section{Discussion}

The purposes of this retrospective case control study were to describe the characteristics of cardiopulmonary indicators (CPI) among patients with normal ventilator weaning response (NVWR) and dysfunctional ventilator weaning response (DVWR) after CABG surgery, to find the differences in characteristics of cardiopulmonary indicators between patients with NVWR and DVWR after CABG surgery, and to build a prediction model for DVWR with significant antecedence. The CPI measurements were collected on an hourly basis for 12 hours postoperatively for cases and controls through a retrospective electronic patient file system (EPF) audit. A purposive sample of 300 subjects was recruited from the medical records of patients who underwent CABG surgery in a Mid-South private hospital, Memphis, TN, from May 2003 to February 2006. This study utilized descriptive and inferential statistical analyses, which were performed through SAS programs including PROC UNIVARIATE, PROC FREQ, PROC GLM, PROC REG, PROC MIXED REPEATED MEASURE ANOVA, and PROC LOGISTIC. The analysis consisted of preplanned comparisons of study variables including DVWR, NVWR, and CPI and post hoc comparisons of selected demographic variables. The independent variable of the study was CPI, which is an hourly time series measurement of selected CPI for 12 consecutive hours after CABG surgery. The outcome variables of this study were DVWR and NVWR. 
The demographic variables included age, sex, BSA, number of grafts, COPD, $\mathrm{CHF}$, and renal failure. The demographic variables were used to describe the study sample as well as included in the post hoc analysis as covariates.

The discussion of the study findings is based on the following research questions explored in the study:

1. What are the characteristics of cardiopulmonary indicators in patients with dysfunctional ventilator weaning response and normal ventilator weaning response?

2. What are the differences in the characteristics of cardiopulmonary indicators in patients with dysfunctional ventilator weaning response and normal ventilator weaning response?

3. What is the best predictive model for dysfunctional ventilator weaning response? The findings of this study based on the above listed research questions are discussed with the past literatures and organized under the following headings, discussion of study variables, discussion on demographic variables, conceptual framework, implications, and conclusion.

\section{Discussion of Study Variables and Findings}

In this section, findings from this research study are discussed under each independent study variable. Each independent variable's findings addressing research question —including description, differences, and prediction modeling — are compared and contrasted with findings from the published literature. 


\section{Cardiopulmonary Indicators and Dysfunctional Ventilator Weaning Response}

Heart rate and dysfunctional ventilator weaning response: This study found that HR is not a significant predictor for DVWR after CABG surgery. The results revealed that there were no significant differences in the hourly mean heart rate between cases and controls except at the first hour. The increase in heart rate among cases during the first hour was consistent with Estenssoro (2005), who reported that weaning from ventilation after CABG surgery involves stress to the heart, which is reflected as increased heart rate during the weaning process (89). However, this finding contrasts with Ely's (1996) report, which indicated that increases in HR are associated with DVWR (36, 90). This contrast finding may be attributable to the nature of the sample. Ely's study was conducted among medical, surgical, and coronary intensive-care-ventilated patients, whereas this study sample was limited to postoperative CABG patients only. Sampling technique might also have contributed to the difference in findings. Ely used a convenient sampling technique that had no exclusion criteria, whereas this study included exclusion criteria to exclude patients receiving chronotropic drugs. This exclusion contributed to the normal distribution of HR between cases and controls; in comparison, there were no significant differences noted. Further, the logistic prediction model revealed that HR is not a significant antecedence to predict DVWR after CABG surgery. Although this retrospective study concluded that HR is not a significant predictor for DVWR, further subgroup analysis based on the rhythm, preoperative treatment with beta-blockers and preoperative heart rate and rhythm may be useful in exploring the nature of heart rate after CABG surgery. 
Mean arterial pressure and dysfunctional ventilator weaning response: In this study, MAP is identified as a significant predictor for DVWR after CABG surgery. The univariate analysis results revealed that there were no significant differences in the hourly mean MAP between cases and controls except at the fifth hour. There were significant differences $(p=0.01)$ in mean MAP at fifth hour between cases and controls. The logistic prediction model revealed that patients who had low MAP had increased risk of developing DVWR after CABG surgery. The odds in favor of DVWR for patients with decrease $10 \mathrm{~mm} / \mathrm{Hg}$ mean MAP were 1.915 times the probability of NVWR, holding all other variables constant. This implies that hypotension increases risk of developing DVWR after CABG surgery. This finding is in contrast with Ely's (1996) report, which indicated that increases in MAP had association with DVWR $(36,90)$. This contrast finding may be attributable to the exclusion criteria of this study, which excluded patients receiving epinephrine, norepineprine, and dopamine. This exclusion resulted in the normally distributed comparable MAP between cases and controls. From these findings, this study concluded that MAP is a significant predictor for DVWR after CABG surgery. Further, prospective studies including subgroup analysis based on the preoperative blood pressure, preoperative medications, postoperative fluid loss, fluid replacements, and treatment with inotrops may be useful to explore and explain the above significant finding.

Central venous pressure and dysfunctional ventilator weaning response: This study found that CVP is not a significant predictor for DVWR after CABG surgery. A 
univariate analysis showed a significant difference between cases and controls in the mean CVP at third hour $(p=0.05)$, seventh hour $(p=0.004)$, eighth hour $(p=0.01)$, ninth hour $(p<0.0001)$, tenth hour $(p=0.05)$, and twelfth hour $(p=0.001)$. This finding implies that patients with DVWR have higher CVP than patients with NVWR. This finding is consistent with the literature which reported increased positive fluid balance reflected in increased CVP associated with prolonged mechanical ventilation (106). Significant differences in mean CVP between cases and controls at the third hour may be explained by the theory that reported changes in intrathoracic fluid volumes in patients after coronary artery bypass grafting after changing the ventilatory pattern from mechanical to spontaneous ventilation revealed that increased preload during weaning process is associated with weaning failure (107).

Although univariate analysis showed significant differences, the logistic prediction model revealed that CVP had no significant value to predict DVWR after CABG surgery - thus, suggesting that CVP is not a significant antecedence to predict the DVWR after CABG surgery. It implies that CVP in patients with DVWR is different from CVP in patients with NVWR; however, the difference is not significant enough to predict the DVWR. The controlled clinical environment may explain this. Clinically CVP is used to detect and treat the fluid status of patients. Measurement of CVP is a guide to assess the preload and fluid status postoperatively. In clinical practice, postoperative CABG surgery patients' fluid status is maintained at negative balance for the first 12 hours through the state of diuresis from the effect of postoperative diuretics in order to prevent pulmonary edema. Further, postoperative $\mathrm{CABG}$ patients with mechanical ventilation are at risk of developing altered preload and fluid status. Mechanical 
ventilation is associated with alterations in intrathoracic pressure; lung volume, and venous return, and changes in intrathoracic fluid volumes during weaning are associated with weaning failure. In patients with impaired cardiac function, the development of pulmonary edema during weaning has been described (58). Although CVP is not a predictive indicator for DVWR, it showed a significant difference between cases and controls indicating that CVP is a clinically sensitive parameter.

Respiratory rate and dysfunctional ventilator weaning response: In this study, $R R$ is identified as a significant predictor for DVWR after CABG surgery. The univariate analysis results revealed that there was no significant difference in the mean RR between cases and controls at the first hour. However, there was a statistically significant difference between cases and controls in mean RR in all the hours except first hour. The lack of differences in the first hour may be explained by the effect of anesthesia, sedations, and ventilator settings, which are common for both cases and controls. In addition, the logistic prediction model in this study revealed that $R R$ is a significant antecedence to predict DVWR. This finding is consistent with literature, which reported that $R R$ is a sensitive indicator to predict the weaning outcome (110). The odds in favor of DVWR for patients with decrease 5 points of mean RR is 2.978 times of the probability of NVWR holding all other variables constant. This finding implies that patients with lower RR are at risk of developing DVWR after CABG surgery. This finding contrasts with past literature, which reported that increased RR is associated with the cardiopulmonary stresses and compensatory effort to combat hypoxia $(36,60,109)$. 
Based on these findings, this study suggests that RR is a significant predictor for DVWR after CABG surgery.

Respiratory rate is a significant predictor for DVWR, as it reflects the neurochemical balance of oxygenation of the patient. Further, it implies that decrease in respiratory rate may be an indication to delay the weaning process. Future prospective studies, including subgroup analysis involving respiratory rate before, during, and after weaning process, may be useful to demonstrate the cause and effect relationship of the respiratory rate in prediction of DVWR. In addition, subgroup analysis including various grades of COPD may be useful to understand the pattern of respiratory rate during postoperative weaning process among these patient populations. Based on the findings, this study concludes that RR is a significant predictor to predict DVWR.

Oxygen saturation and dysfunctional ventilator weaning response: This study found that $\mathrm{SPO}_{2}$ is not a significant predictor for DVWR after CABG surgery. The univariate analysis results revealed that there was no significant difference in the hourly mean of $\mathrm{SPO}_{2}$ between cases and controls except fourth, fifth, and sixth hour. This finding may reflect the ventilator support in maintaining oxygenation postoperatively in both cases and controls. However, there was a significant difference between cases and controls in mean $\mathrm{SPO}_{2}$ at fourth hour $(\mathrm{p}=0.01)$, fifth hour $(\mathrm{p}=0.01)$, and sixth hour $(\mathrm{p}=$ 0.05). This finding is consistent with the past literature which reported that differences in $\mathrm{SPO}_{2}$ were associated with $\operatorname{PMV}(59,86)$. There was a significant decline in the controls noted after the fourth hour, which may be due to the weaning process and extubation in controls, whereas cases continued to receive ventilator support to maintain 
the oxygenation. To understand this trend and significance of this trend, a subgroup analysis may be conducted in future research comparing cases and controls in the context of $\mathrm{SPO}_{2}$ and ventilation hours. Further, the logistic regression prediction model revealed that $\mathrm{SPO}_{2}$ is not a significant antecedence to predict DVWR after CABG surgery.

Cardiac output dysfunctional ventilator weaning response: $\mathrm{In}$ this study, $\mathrm{CO}$ was identified as a significant predictor for DVWR after CABG surgery. There was a significant difference in the hourly mean of $\mathrm{CO}$ between cases and controls except in the ninth, tenth, and eleventh hours. It can be inferred that $\mathrm{CO}$ was significantly lower in patients with DVWR when compared with patients with NVWR. This finding is consistent with the literature, which reported that low CO is associated with PMV after CABG surgery $(20,37)$. The odds in favor of DVWR for patients with decrease in mean CO by 2 points is 1.943 times of the probability of NVWR holding all other variables as constant. This finding implies that patients with low $\mathrm{CO}$ are at risk of developing DVWR after CABG surgery. This finding is consistent with findings of Estenssoro (2005) who reported that low cardiac output has the highest association with DVWR (89). From these findings, this study suggests that $\mathrm{CO}$ is a significant predictor for DVWR after CABG surgery.

Increase or decrease in the cardiac output reflects the cardiac function involving rate, rhythm, contractility, volume, and systemic vascular resistance. The harmonization of these collective functions produces the cardiac output through the electromechanical action of the heart. This study finding suggests that decreased cardiac output is a significant predictor for DVWR after CABG surgery. Postoperatively, CO may be 
decreased by various factors, including blood or fluid loss during and after surgery, temporary myocardial depression after surgery, excessive diuresis, decreased fluid intake, and decreased systemic vascular resistance due to autonomic neuropathy, etc. Further prospective studies are needed to establish cause and effect relationship for DVWR. Exploring the causative factors for decreased cardiac output may be useful to find the cause and effect relationship between decreased CO and DVWR after CABG surgery. In addition, including the patients with the treatment of inotropics and performing the subgroup analysis may be helpful to understand the various causes and treatment of decreased cardiac output after CABG surgery.

\section{Cardiac index and dysfunctional ventilator weaning response: This study}

found that $\mathrm{CI}$ is not a significant predictor for DVWR after CABG surgery. The univariate analysis results revealed that there is significant difference in the hourly mean of CI between cases and controls in first, fifth, sixth, eighth and twelfth hours, with the CI of the control group significantly higher. This finding implies that the patients who had lower CI developed DVWR. This finding is consistent with past literature that reported an alteration in cardiac index during weaning was found to have association with weaning failure $(15,76-78)$. However, the logistic prediction model revealed that CI is not a significant antecedence to predict the DVWR after CABG surgery. This finding may be explained in consideration with BSA. Cardiac index is a measurement which is adjusted for BSA. 


\section{Pulmonary artery diastolic pressure and dysfunctional ventilator weaning}

response: In this study, PAD is identified as a significant predictor for DVWR after CABG surgery. There is significant difference in the hourly mean of PAD between cases and controls in all the hours except first and eighth hour. The odds (3.640) in favor of DVWR for patients with an increase in mean PAD implies that the patients with high PAD pressure are at the risk of developing DVWR after CABG surgery. This finding affirms past literature, which reported that PAD pressures may be used as a sensitive predictor of DVWR since pulmonary hypertension is associated with weaning failure after heart surgery (112-116).

Clinically, elevated PAD is seen in association with various conditions that lead to pulmonary artery hypertension. The common associated conditions include COPD, CHF, and left ventricular hypertrophy, pulmonary artery constriction, pulmonary hypoxemia, acute respiratory distress syndromes, and pulmonary edema. Knowledge based on this retrospective study finding indicates that elevated PAD is a significant predictor for DVWR. However, further analyzing the causative factors through a prospective study may reveal the cause effect relationship of elevated PAD with DVWR after CABG surgery. In addition, a subgroup analysis including subjects with COPD and CHF may be helpful in exploring the nature of PAD increase after CABG surgery. Further preoperative and postoperative comparison of the PAD may be necessary to demonstrate the changes in PAD in DVWR subjects during weaning process. Based on the finding, this study concludes that elevated PAD is a significant predictor for DVWR after CABG surgery. From this knowledge it can be implied that PAD may be included in the weaning protocol as it is s significant predictor of DVWR. Clinically, current 
weaning protocols contain the guidelines to treat low PAD after CABG surgery; however, there are no guidelines established to treat increased PAD after CABG surgery. Establishing guidelines to treat increased PAD after CABG surgery may be tested, followed by prospective studies to establish treatment guidelines for DVWR after CABG surgery.

\section{Pulmonary artery systolic pressure and dysfunctional ventilator weaning}

response: In this study, PASP was identified as a significant predictor for DVWR after CABG surgery. There was no significant difference between cases and controls in the hourly mean of PASP in all 12 consecutive hours. This finding supports past theory that suggested changes in pulmonary artery pressures are sensitive indicators of altered cardiopulmonary and fluid status within the circulatory system. Elevated pulmonary artery pressures are the indication of pulmonary hypertension, which is directly associated with DVWR (58). In contrast, the logistic regression prediction model in this study revealed that patients those who had lower PASP developed DVWR. The odds in favor of DVWR for patients with decrease in mean PASP by $10 \mathrm{~mm} / \mathrm{hg}$ were 3.053 times the probability of NVWR, holding all other variables constant. This implies that the patients with low PASP are at risk of developing DVWR after CABG surgery. This finding implies that PASP is a significant antecedence to predict DVWR after CABG surgery. Predictive ability of low PASP for DVWR may be explained, as PASP is an indicator of the filling pressure. Literature suggests that higher filling pressures are required during the first 4 hours after $\mathrm{CABG}$ surgery to enhance the coronary perfusion. This finding also affirms past literature, which reported that PASP pressure may be used 
as a sensitive predictor of DVWR as pulmonary hypertension is associated with weaning failure after heart surgery (112-116).

When the PASP is lower there is higher chance of developing DVWR. Decrease in PASP may be an indication of either decreased right ventricular preload or decreased pulmonary vascular resistance. The majority of the subjects among the DVWR sample contained preoperative morbidity such as COPD and CHF. In patients with COPD, CHF may tend to have higher PASP preoperatively and their hemodynamic homeostasis may adapt to the higher filling pressures preoperatively. This finding needs further exploration by doing a comorbidity specific subgroup analysis involving prospective studies in order to establish the cause and effect relationship. Clinically, increased PASP is treated postoperatively; however, there is no protocol to treat decreased PASP. Developing a protocol to low PASP after CABG surgery may be beneficial.

\section{Mixed venous oxygen saturation and dysfunctional ventilator weaning}

response: This study found that $\mathrm{SVO}_{2}$ is not a significant predictor for DVWR after CABG surgery. The results revealed no significant difference between cases and controls in the hourly mean of $\mathrm{SVO}_{2}$ in all hours except eleventh and twelfth hours. Past literature indicated that a progressive decrease in $\mathrm{SVO}_{2}$ during the weaning process is reported as associated with weaning failure $(87,88)$. In addition, some studies have found an association between a decrease in $\mathrm{SVO}_{2}$ after extubation and reintubation $(37,73,108)$. In contrast, in this study, $\mathrm{SVO}_{2}$ was significantly higher in patients with DVWR at eleventh and twelfth hour. However, the logistic prediction model in this study revealed that $\mathrm{SVO}_{2}$ is not a significant antecedence to predict DVWR after CABG surgery. This 
finding contrasts with past literature. This contrast finding may need further investigation to interpret the trend. Information such as body temperature and hemoglobulin levels are needed to understand this trend of increased $\mathrm{SVO}_{2}$ among cases. Increased $\mathrm{SVO}_{2}$ may be an indication of decreased peripheral oxygen conception due to hypothermia or peripheral shunting. Further studies are needed to explore the characteristics of this variable in the context of hypothermia and anemia.

\section{Discussion of Demographic and Clinical Variables}

Age and dysfunctional ventilator weaning response: This research study's findings revealed that age distribution had no significant difference between cases and controls. However, age had a significant effect on CPI, such as HR ( $p=0.008)$, MAP $(\mathrm{p}=0.0028), \mathrm{SPO}_{2}(\mathrm{p}=0.042), \mathrm{CO}(\mathrm{p}=0.015), \mathrm{CI}(\mathrm{p}=0.016)$, and PAD $(\mathrm{p}=0.04)$. These findings were consistent with the findings of Legare, et al. (2001) (14). Although age had a significant effect on the above CPI, a logistic regression analysis revealed that age did not have significant predictive value for DVWR after CABG surgery. This finding implies that age had no significant effect on DVWR after CABG surgery. This finding was consistent with Bezanson, et al. (2001) who reported that age has no effect on PMV after CABG surgery (12). The finding also agrees with current literature which indicates that increased age is not associated with PMV after CABG surgery $(1,2,7,12)$.

Sex and dysfunctional ventilator weaning response: The distribution of sex between cases and controls showed a significant $(p=0.01)$ difference. However, the odds 
ratio for sex was less than one, which implies that sex is not a significant risk factor for developing DVWR after CABG surgery. This finding is consistent with Athanasiou, et al., (2003), who reported that gender was not found to be an independent predictor for prolonged ventilation after CABG surgery (189). In addition, this study finding revealed that gender had significant effects on CPI such as MAP $(p=0.0012), C O(p<0.0001)$, and CI ( $\mathrm{p}<0.0001)$. Although, sex had a significant effect on these CPI, a logistic regression model revealed that gender had no significant predictive value for DVWR after CABG surgery. This finding contrasts with the findings of Legare, et al.(2001) who reported that females are at risk of developing DVWR after CABG surgery (14). This contrast finding may be explained by the sample size. Legare, et al.used a large sample size of 1,800 subjects when compared with this study, which had smaller sample size involving 300 subjects. The significance of female gender may be visible more in the larger sample size. Legare, et al. included all the subjects without exclusion criteria; this may be another factor that could contribute to the contrast finding. Although these study findings revealed that females are not at risk of developing DVWR, it is imperative to add sex as an independent variable in future studies in consideration with physiological differences between the genders.

\section{Renal failure and dysfunctional ventilator weaning response: This study} found that renal failure is not a significant predictor for DVWR after CABG surgery. There was no significant difference in the prevalence of renal failure between cases and controls in this study sample. A repeated measure ANOVA analysis revealed that renal failure had a significant $(\mathrm{p}=0.019)$ effect on $\mathrm{SPO}_{2}$. This finding is consistent with the literature, which indicates that renal anemia predisposes to alterations in $\mathrm{SPO}_{2}$ because it 
induces functional and organic alterations of cardiac-circulatory function (131). However, a logistic regression model in this study indicated that renal failure is not a significant predictive factor for DVWR after CABG surgery.

\section{Chronic obstructive pulmonary disease and dysfunctional ventilator weaning}

response: In this study, COPD is identified as a significant predictor for DVWR after CABG surgery. The prevalence of COPD was found to make a significant $(\mathrm{p}<0.0001)$ difference between cases and controls. The odds ratio of COPD was 5.11. It implies that the relative risk of patients with COPD developing DVWR was 5.11 times higher than for patients without COPD. This finding was consistent with Legare, et al.(2001) who indicated that COPD is a significant preoperative risk factor for PMV after CABG surgery (14). In addition, this study's finding revealed that COPD had no significant effect on any of the selected CPI, which implies that COPD is an independent risk factor for DVWR. This finding is consistent with the literature that indicates that COPD is associated with DVWR (26, 177-180). Further, a regression model of this study affirmed that COPD is a significant $(\mathrm{p}=0.0003)$ predictor for DVWR after CABG surgery. The odds in favor of DVWR for patients with COPD were 5.466 times higher than for patients without COPD, holding all other variables constant. From these findings, this study concludes that COPD is a significant predictor for DVWR after CABG surgery.

\section{Congestive heart failure and dysfunctional ventilator weaning response: In} this study, CHF is identified as a significant predictor for DVWR after CABG surgery. Prevalence of CHF resulted in a significant $(\mathrm{p}=0.0003)$ difference between cases and 
controls. The relative risk (odds ratio) of CHF was 6.75 for developing DVWR. This finding was consistent with the literature that indicated an association of congestive heart failure (CHF) with PMV after CABG surgery $(6,7,12,20,21,68)$. In this study, CHF had significant effect on CPI $(p=0.0034)$. This finding is consistent with the literature that indicates the effect of CHF on PASP is due to the cardiopulmonary dynamics, explained by Boldt and Hempelmann (2001) as follows: The pathophysiological changes due to left ventricular failure are associated with the increased pulmonary pressures and decreased cardiac out put resulting in delayed ventilator weaning. In addition, cardiac surgery depress the myocardial pump function (80). Other literature explained that right heart failure is associated with an increased right atrial pressure, and impeding venous return and venous congestion alter hemodynamics and pulmonary artery pressures (125, 126).

Furthermore, a logistic regression model in this study indicated that CHF was a significant $(p=0.04)$ predictor for DVWR after CABG surgery. The odds in favor of DVWR for patients with CHF were 3.930 times higher than for patients without CHF, holding all other variables constant. From these findings, this study concluded that CHF was a significant predictor for DVWR after CABG surgery.

\section{Number of grafts and dysfunctional ventilator weaning response: This} research study revealed that there was no significant difference in the number of grafts between cases and controls. In addition, the number of grafts had a significant $(p=0.05)$ effect on mean PASP. Past literature indicated that the number of grafts was associated with PMV after CABG surgery $(6,7,12,20,21,68)$. However, the logistic regression 
model in this study revealed that number of grafts was not a significant predictive factor for DVWR after CABG surgery.

Body surface area and dysfunctional ventilator weaning response: In this study, there was no significant difference in mean BSA between cases and controls. Repeated measure ANOVA analysis revealed that BSA had significant effect on CPI such as CVP ( $p=0.04), \mathrm{RR}(\mathrm{p}=0.0028), \mathrm{SPO}_{2}(\mathrm{p}=0.0034), \mathrm{CO}(\mathrm{p}<0.0001), \mathrm{CI}(\mathrm{p}<$ $0.0001)$, and PASP $(p=0.0059)$. Although BSA had significant effect on abovementioned CPI, the logistic regression model indicated that BSA is not a significant predictive factor for DVWR. This finding is consistent with the findings of Parlow (2006) reported that obesity is not associated with extubation failure after CABG surgery (190). However, including the body mass index in future prospective studies may be beneficial.

This study was guided by a conceptual framework, which was built upon past literature and theories. The conceptual model which contains significant predictors depicted in Figure 5.1 describes the association of the risk factors and CPI with DVWR and NVWR after CABG surgery. It explains that cardiac surgery is followed by ventilator weaning in postoperative critical care. Postoperatively, the mechanical ventilation and hemodynamic stabilization therapies help to restore cardiopulmonary function. The outcome of those therapies is either normal ventilator weaning response (NVWR) or DVWR. The above-mentioned outcomes influence the CPI. The changes in CPI reflect 


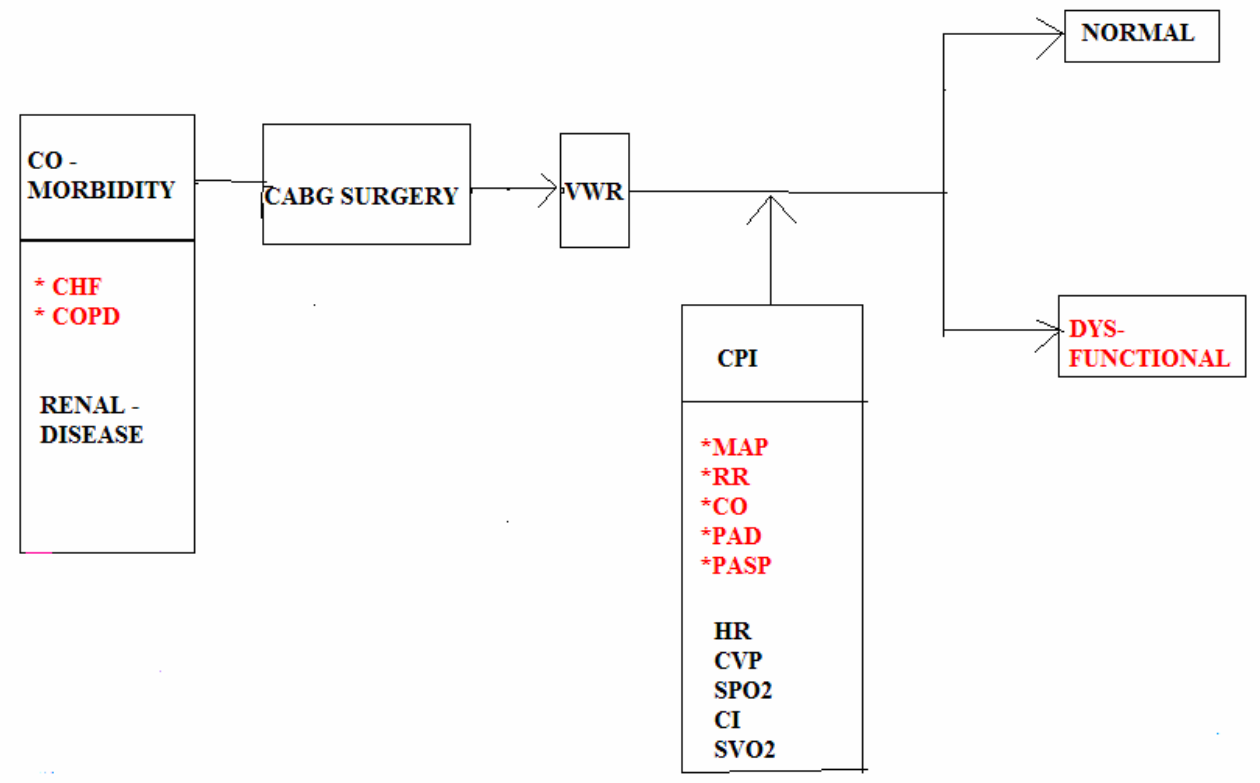

Figure 5.1. Conceptual Model Indicating Significant Predictors of DVWR.

Note. * Significant predictor for dysfunctional ventilator weaning response $\mathrm{CHF}$ : congestive heart failure

COPD: chronic obstructive pulmonary disease

CABG: coronary artery bypass surgery

VWR: ventilator weaning response

CPI: cardiopulmonary indicators

HR: heart rate

MAP: mean arterial pressure

CVP: central venous pressure

$\mathrm{RR}$ : respiratory rate

$\mathrm{SPO}_{2}$ : oxygen saturation

CO: cardiac output

$\mathrm{CI}$ : cardiac index

PAD: pulmonary diastolic pressure

PASP: pulmonary artery systolic pressure

$\mathrm{SVO}_{2}$ : mixed venous oxygen saturation 
changes in the patient's condition. Therefore, it can be assumed that changes in CPI precede DVWR. Thus CPI was set to predict NVWR and DVWR, which are associated with restored cardiopulmonary function and impaired cardiopulmonary function. In this study, the temporal associations of CPI and comorbidities were tested and their predictive value was assessed.

This study supported the research assumption which indicated that the clinical changes that preceded DVWR reflected on the changes on CPI. The research questions formulated guided by the conceptual framework were explored and the study results supported the temporal relationship of the variables as antecedence for DVWR after CABG surgery.

This study revealed that co-morbid risk factors including COPD and CHF had a significant predictive value for DVWR after CABG surgery. CPI, including MAP, RR, CO, PAD, and PASP, also had a significant predictive value for DVWR after CABG surgery. These findings of this study have a foundation in past theory and are well supported by past literature.

The theoretical underpinning of the conceptual framework has significance in predicting DVWR after CABG surgery. Among the selected antecedence-including comorbidities and CPI—many of them were found significant in predicting DVWR, which can be explained by the conceptual framework. The statistical model of this study revealed the significant co-morbidities that have predictive value for DVWR after CABG surgery, including COPD and CHF. In addition, significant CPI's predictive value was explained by the conceptual framework and tested through the statistical model of this study. Accordingly, CPI such as MAP, RR, CO, PAD, and PASP can be used to predict 
DVWR after CABG surgery through this model. The above-mentioned significant antecedence was tested through logistic regression statistical model.

The theory derived from this study offers the possibility of explaining DVWR after CABG surgery. The theory was found testable through statistical logistic model. All the concepts described in the conceptual framework were found measurable and were subjected for statistical analysis, and significance was tested through statistical hypothesis. The empirical findings of this study supported the conceptual framework and the model.

In conclusion, this research study identified the significant antecedence to predict DVWR after CABG surgery. Statistical significance was tested through a logistic prediction model. The significant predictors included COPD, CHF, mean MAP, mean RR, mean CO, mean PAD, and mean PASP. Therefore, this study concluded that the abovementioned significant predictors may be used to predict DVWR after CABG surgery.

\section{Implications}

This study's results have implications for the theory, practice, education, and research involving postoperative care after $\mathrm{CABG}$ surgery from the perspectives of early detection, prevention, and treatment of DVWR. The knowledge acquired through this study includes antecedence such as $\mathrm{COPD}, \mathrm{CHF}$, mean MAP, mean RR, mean CO, mean PAD, and mean PASP that were found significant in the prediction of DVWR after CABG surgery. This specific knowledge can be used to benefit the fields of critical care medicine, critical care nursing, and critical care administration in the aspects of theory, 
practice, education, research, quality improvement, and cost-effective care in postoperative critical care after CABG surgery.

Theory and practice: The findings of this research study have an implication for critical care medicine in the aspects of prevention, treatment, and health promotion. They may contribute to the prevention of ICU mortality and morbidity following CABG surgery. The finding of the significant predictors of DVWR after CABG surgery has added new knowledge to critical care medicine, critical care nursing, and respiratory medicine. Introduction of this new knowledge in the discipline's practice may enable the prevention of DVWR after CABG surgery. The description of the trends of the significant predictors has added new knowledge to the respective disciplines, which may in turn help in planning postoperative care for patients in the ICU who had CABG surgery. This knowledge may also aid in preventing premature weaning trials and in providing early treatment, which in turn can prevent mortality and morbidity associated with CABG surgery.

This study benefits critical care nursing, as it adds new knowledge to the discipline. The knowledge of significant antecedence for DVWR would contribute to the critical care nursing practice in the aspects of prevention of complications and providing quality care through hemodynamic monitoring and assessment skills. Postoperative ventilator weaning is the major task of critical care nursing following CABG surgery. The critical care nurse plays an important role in the postoperative monitoring of CPI, hemodynamic stabilization, assessing the patient's readiness for weaning, and in preventing postoperative complications. The trends of the CPI and prediction model 
derived from this research study could provide a reliable tool to guide the practice of monitoring, assessment, and ventilator weaning practice after CABG surgery.

This research study may contribute to critical care nursing education through the addition of new knowledge to nursing theory. The specific findings, such as trends of CPI and significant antecedence of DVWR, may contribute to the development of the nursing process for caring for patients after CABG surgery.

The findings of this research study may contribute to the quality of care in critical care nursing and medicine through the implications for patient safety after CABG surgery. The identification of reliable predictors for DVWR, the trends of CPI for DVWR, and the prediction model may help to identify a possible DVWR before it occurs, thereby it may help in preventing premature ventilator weaning trials that subject vulnerable patients to the risk of developing hypoxia, myocardial ischemia, and the complications that follow. In addition, this research study has implications for the quality improvement through the development of weaning protocols and policy after CABG surgery.

Research: This research study contributes to critical care research. The new knowledge derived from this study opens new avenues for future research, such as the development of a preoperative risk assessment tool using the significant antecedence. The knowledge of COPD and CHF has significant predictive value for DVWR after CABG surgery and can be used to study the comparative preoperative risk factors. In addition, interventional studies can be conducted with preoperative teaching and treatments among above risky populations. 
Further, studying the perspectives of development of a preoperative teaching tool and interventions focused on high-risk individuals may be useful. Next, incorporation of significant antecedence in weaning protocols and development of patient specific and risk specific weaning protocols may open the avenue for quality improvement of evidencebased projects. Testing the effectiveness of the weaning protocols, performance assessment and comorbidity-specific weaning practice may be planned as an evaluation research. In addition, this study may be conducted with a larger sample involving multiple sites before generalizing the results and to test the reliability and validity of the findings. Furthermore, the research design, methods, and analysis adopted and tested in this study could aid in guiding future research addressing similar problems in other populations, settings, and disciplines.

The findings of this research study may also contribute to cost-effective care in critical care after CABG surgery. Including ICU length of stay as a variable may help to study the cost involved in treating high-risk population. The significant antecedence may help predict and prevent DVWR after CABG surgery, which in turn can result in reduced ICU length of stay after CABG surgery. Thereby, it can reduce the postoperative ICU cost.

Based on this study's findings, the following further research questions arise:

1. This study found that there was a significant effect of time on RR, which leads to the question regarding whether there are differences in the effect of time on RR between cases and controls. 
2. This study found that there was a significant effect of the number of grafts on RR and $\mathrm{SPO}_{2}$. This leads to the question regarding whether there are differences in the effect of number of grafts on RR and $\mathrm{SPO}_{2}$.

3. This study found that there were differences in the effects of time on PAD, which leads to the question about what are the differences in the effect of time on PAD.

4. This study found significant differences in the means of certain CPI including RR, $\mathrm{CO}, \mathrm{CI}, \mathrm{PAD}$, and $\mathrm{SPO}_{2}$, that lead to the question about what are the differences in trends of above mentioned CPI.

5. This study found that COPD has a significant predictive value for DVWR, which leads to questions such as:

- What are the preoperative pulmonary indicators (volumes and compliances) that exist with patients with COPD?

- What are the preoperative treatments that can maximize the lung functions to enhance normal weaning?

- What are the other attributes that coexist with this disease condition that affect the weaning outcome?

- What is the clinical model that can guide in the postoperative management of patients with COPD?

\section{Conclusion}

This retrospective case control study set out to find the values of CPI in the prediction of DVWR after CABG surgery. The study was guided by three research questions, which included describing the characteristics of CPI among patients with 
NVWR and DVWR after CABG surgery, finding the differences in characteristics of cardiopulmonary indicators between patients with NVWR and DVWR after CABG surgery, and building a prediction model for DVWR with significant antecedence. A retrospective case control study was utilized with a time series design using a sample consisting of 300 subjects who underwent CABG surgery. The results of the study were derived from the statistical analysis by SAS performing a univariate analysis, repeated measure ANOVA mixed procedure, and logistic regression analysis. The results of this study revealed significant antecedence to predict DVWR after CABG surgery, which includes COPD, CHF, MAP, RR, CO, PAD, and PASP. Therefore, this study concludes with a recommendation that the above-mentioned significant antecedence may be used to predict DVWR after CABG surgery in critical care. Further prospective studies are needed to establish the cause and effect relationship of these predictors to DVWR after CABG surgery. 


\section{LIST OF REFERENCES}

1. Branca P, McGaw P, Light R. Factors associated with prolonged mechanical ventilation following coronary artery bypass surgery. Chest 2001;119(2):537-46.

2. Brucek PJ, Straka Z, Vanek T, Jares M. Less invasive cardiac anesthesia: an ultrafast-track procedure avoiding thoracic epidural analgesia. Heart Surg Forum 2003;6(6):E107-10.

3. Capdeville M, Lee JH, Taylor AL. Effect of gender on fast-track recovery after coronary artery bypass graft surgery. J Cardiothorac Vasc Anesth 2001;15(2):14651 .

4. Nichols JD, Stammers AH, Kmiecik SA, Liu JL, Kohtz RJ, Mills NJ, et al. Effects of increasing $\mathrm{FiO} 2$ on venous saturation during cardiopulmonary bypass in the swine model. J Extra Corpor Technol 2002;34(2):118-24.

5. Sulzer CF, Chiolero R, Chassot PG, Mueller XM, Revelly JP. Adaptive support ventilation for fast tracheal extubation after cardiac surgery: a randomized controlled study. Anesthesiology 2001;95(6):1339-45.

6. Alexander WA, Cooper JR, Jr. Preoperative risk stratification identifies low-risk candidates for early extubation after aortocoronary bypass grafting. Tex Heart Inst $\mathbf{J}$ 1996;23(4):267-9.

7. Bezanson JL, Weaver M, Kinney MR, Waldrum M, Weintraub WS. Presurgical risk factors for late extubation in Medicare recipients after cardiac surgery. Nurs Res 2004;53(1):46-52.

8. Doering LV, Esmailian F, Laks H. Perioperative predictors of ICU and hospital costs in coronary artery bypass graft surgery. Chest 2000;118(3):736-43.

9. Guller U, Anstrom KJ, Holman WL, Allman RM, Sansom M, Peterson ED. Outcomes of early extubation after bypass surgery in the elderly. Ann Thorac Surg 2004;77(3):781-8.

10. Tokmakoglu H, Farsak B, Gunaydin S, Kandemir O, Aydin H, Yorgancioglu C, et al. Effectiveness of intraaortic balloon pumping in patients who were not able to be weaned from cardiopulmonary bypass after coronary artery bypass surgery and mortality predictors in the perioperative and early postoperative period. Anadolu Kardiyol Derg 2003;3(2):124-8.

11. Modine T, Decoene C, Al-Ruzzeh S, Athanasiou T, Poivre P, Pol A, et al. Dobutamine improves thoracic aortic blood flow during off-pump coronary artery bypass surgery: results of a prospective randomised controlled trial. Eur J Cardiothorac Surg 2005;27(2):289-95.

12. Bezanson JL, Deaton C, Craver J, Jones E, Guyton RA, Weintraub WS. Predictors and outcomes associated with early extubation in older adults undergoing coronary artery bypass surgery. Am J Crit Care 2001;10(6):383-90.

13. Habib RH, Zacharias A, Engoren M. Determinants of prolonged mechanical ventilation after coronary artery bypass grafting. Ann Thorac Surg 1996;62(4):1164-71. 
14. Legare JF, Hirsch GM, Buth KJ, MacDougall C, Sullivan JA. Preoperative prediction of prolonged mechanical ventilation following coronary artery bypass grafting. Eur J Cardiothorac Surg 2001;20(5):930-6.

15. Nickerson NJ, Murphy SF, Davila-Roman VG, Schechtman KB, Kouchoukos NT. Obstacles to early discharge after cardiac surgery. Am J Manag Care 1999;5(1):2934.

16. Plumer H, Markewitz A, Marohl K, Bernutz C, Weinhold C. Early extubation after cardiac surgery: a prospective clinical trial including patients at risk. Thorac Cardiovasc Surg 1998;46(5):275-80.

17. Serrano N, Garcia C, Villegas J, Huidobro S, Henry CC, Santacreu R, et al. Prolonged intubation rates after coronary artery bypass surgery and ICU risk stratification score. Chest 2005;128(2):595-601.

18. Spivack SD, Shinozaki T, Albertini JJ, Deane R. Preoperative prediction of postoperative respiratory outcome. Coronary artery bypass grafting. Chest 1996;109(5):1222-30.

19. Doering LV, Imperial-Perez F, Monsein S, Esmailian F. Preoperative and postoperative predictors of early and delayed extubation after coronary artery bypass surgery. Am J Crit Care 1998;7(1):37-44.

20. Yende S, Wunderink R. Validity of scoring systems to predict risk of prolonged mechanical ventilation after coronary artery bypass graft surgery. Chest 2002;122(1):239-44.

21. Yende $\mathrm{S}$, Wunderink $\mathrm{R}$. Causes of prolonged mechanical ventilation after coronary artery bypass surgery. Chest 2002;122(1):245-52.

22. Ferguson JJ, 3rd, Cohen M, Freedman RJ, Jr., Stone GW, Miller MF, Joseph DL, et al. The current practice of intra-aortic balloon counterpulsation: results from the Benchmark Registry. J Am Coll Cardiol 2001;38(5):1456-62.

23. Insler SR, O'Connor MS, Leventhal MJ, Nelson DR, Starr NJ. Association between postoperative hypothermia and adverse outcome after coronary artery bypass surgery. Ann Thorac Surg 2000;70(1):175-81.

24. Anderson RJ, O'Brien M, MaWhinney S, VillaNueva CB, Moritz TE, Sethi GK, et al. Renal failure predisposes patients to adverse outcome after coronary artery bypass surgery. VA Cooperative Study \#5. Kidney Int 1999;55(3):1057-62.

25. Leon-Valles M, Suarez-Pinilla MA, Abad-Diez JM, Carreras-Gargallo L, Trujillano-Cabello JJ, Sanz-Gonzalo T. Identification of patients with a high risk of needing prolonged mechanical ventilation after coronary surgery. Rev Esp Anestesiol Reanim 1996;43(3):82-8.

26. Currey J, Botti M. Naturalistic decision making: a model to overcome methodological challenges in the study of critical care nurses' decision making about patients' hemodynamic status. Am J Crit Care 2003;12(3):206-11.

27. Nakai Y, Bando M, Nishimura T, Kataoka Y. Coronary artery bypass surgery in patients aged 75 years or older. Kyobu Geka 1995;48(6):477-80.

28. Westaby S, Pillai R, Parry A, O'Regan D, Giannopoulos N, Grebenik K, et al. Does modern cardiac surgery require conventional intensive care? Eur J Cardiothorac Surg 1993;7(6):313-8; discussion 318. 
29. Kollef M, Wragge T, Pasque C. Determinants of mortality and multiorgan dysfunction in cardiac surgery patients requiring prolonged mechanical ventilation. Chest 1995;107:1395-1401.

30. Kollef MH, Horst HM, Prang L, Brock WA. Reducing the duration of mechanical ventilation: three examples of change in the intensive care unit. New Horiz 1998;6(1):52-60.

31. Kollef MH, Silver P. Ventilator-associated pneumonia: an update for clinicians. Respir Care 1995;40(11):1130-40.

32. Vijay V, McCusker K. Recent advances in biocompatible surface-modifying additives for cardiopulmonary bypass. Perfusion 2003;18 Suppl 1:41-5.

33. Vijay V, Gold J. Late Complications of Cardiac Surgery. In: Cohn L, Edmunds LJ, editors. Cardiac Surgery in the Adult. New York: McGraw-Hill; 2003. p. 521-537.

34. Suematsu Y, Sato H, Ohtsuka T, Kotsuka Y, Araki S, Takamoto S. Predictive risk factors for delayed extubation in patients undergoing coronary artery bypass grafting. Heart Vessels 2000;15(5):214-20.

35. Bardell T, Legare JF, Buth KJ, Hirsch GM, Ali IS. ICU readmission after cardiac surgery. Eur J Cardiothorac Surg 2003;23(3):354-9.

36. Ely EW, Baker AM, Dunagan DP, Burke HL, Smith AC, Kelly PT, et al. Effect on the duration of mechanical ventilation of identifying patients capable of breathing spontaneously. N Engl J Med 1996;335(25):1864-9.

37. Meade M, Guyatt G, Cook D, Griffith L, Sinuff T, Kergl C, et al. Predicting success in weaning from mechanical ventilation. Chest 2001;120(6 Suppl):400S-24S.

38. Schonhofer B. Predictors of weanability. Monaldi Arch Chest Dis 2000;55(4):33944.

39. Suematsu Y, Sato H, Ohtsuka T, Kotsuka Y, Araki S, Takamoto S. Predictive risk factors for pulmonary oxygen transfer in patients undergoing coronary artery bypass grafting. Jpn Heart J 2001;42(2):143-53.

40. Rady MY, Ryan T. Perioperative predictors of extubation failure and the effect on clinical outcome after cardiac surgery. Crit Care Med 1999;27(2):340-7.

41. Epstein SK, Ciubotaru RL. Independent effects of etiology of failure and time to reintubation on outcome for patients failing extubation. Am J Respir Crit Care Med 1998;158(2):489-93.

42. Epstein SK, Ciubotaru RL. Influence of gender and endotracheal tube size on preextubation breathing pattern. Am J Respir Crit Care Med 1996;154(6 Pt 1):164752.

43. Epstein SK. Etiology of extubation failure and the predictive value of the rapid shallow breathing index. Am J Respir Crit Care Med 1995;152(2):545-9.

44. Epstein SK. Extubation failure: an outcome to be avoided. Crit Care 2004;8(5):3102 .

45. Rothaar RC, Epstein SK. Extubation failure: magnitude of the problem, impact on outcomes, and prevention. Curr Opin Crit Care 2003;9(1):59-66.

46. Afessa B, Hogans L, Murphy R. Predicting 3-day and 7-day outcomes of weaning from mechanical ventilation. Chest 1999;116(2):456-61.

47. Rothenburger M, Soeparwata R, Deng MC, Schmid C, Berendes E, Tjan TD, et al. Prediction of clinical outcome after cardiac surgery: the role of cytokines, endotoxin, and anti-endotoxin core antibodies. Shock 2001;16 Suppl 1:44-50. 
48. Keinanen O, Takala J, Kari A. Continuous measurement of cardiac output by the Fick principle: clinical validation in intensive care. Crit Care Med 1992;20(3):360-5.

49. Poelaert J, Heerman J, Schupfer G, Moerman A, Reyntjens K, Roosens C. Estimation of myocardial performance in $\mathrm{CABG}$ patients. Acta Anaesthesiol Scand 2004;48(8):973-9.

50. Kruger AD, Francke A, Emmrich K. Enoximone--clinical experiences in heart surgery. Anaesthesiol Reanim 1996;21(3):60-8.

51. Epstein SK. Decision to extubate. Intensive Care Med 2002;28(5):535-46.

52. Bezanson JL, Strickland OL, Kinney MR, Weintraub WS. Assessing data adequacy for clinical research: reliability and validity of a surgical database. J Nurs Meas 2002;10(2):155-64.

53. Clavey M, Mattei MF, Hubert T, Peiffert B, Retournard JL, Zamorano J, et al. Value of continuous venous blood oxygen measurement during external circulatory assistance. Ann Fr Anesth Reanim 1990;9(1):83-6.

54. SPO2.COM. SPO2.COM ${ }^{\mathrm{TM}}$ vs. Nellcor ${ }^{\circledR}$ Oximax ${ }^{\circledR}$ SpO2 sensors* 2004 [cited 2005 March 30]; Available from: http://www.spo2.com/white\%20paper.htm

55. Gimenez A, Fernandez-Reyes I, Marin B, Alvarez MD, Andorra M, Duque FJ, et al. Validation in Spain of the dysfunctional ventilatory response to weaning. Enferm Intensiva 1997;8(3):121-8.

56. Gimenez AM, Serrano P, Marin B. Clinical validation of dysfunctional ventilatory weaning response: the Spanish experience. Int J Nurs Terminol Classif 2003;14(2):53-64.

57. Logan J, Jenny J. Deriving a new nursing diagnosis through qualitative research: dysfunctional ventilatory weaning response. Nurs Diagn 1990;1(1):37-43.

58. Thelan Lynne A. Critical care nursing diagnosis and management. Third ed. Philadelphia: Mosby; 1998.

59. Noll ML, Byers JF. Usefulness of measures of Svo2, Spo2, vital signs, and derived dual oximetry parameters as indicators of arterial blood gas variables during weaning of cardiac surgery patients from mechanical ventilation. Heart Lung 1995;24(3):220-7.

60. Kjaergaard S, Rees SE, Gronlund J, Nielsen EM, Lambert P, Thorgaard P, et al. Hypoxaemia after cardiac surgery: clinical application of a model of pulmonary gas exchange. Eur J Anaesthesiol 2004;21(4):296-301.

61. Andrejaitiene J, Sirvinskas E, Bolys R. The influence of cardiopulmonary bypass on respiratory dysfunction in early postoperative period. Medicina (Kaunas) 2004;40 Suppl 1:7-12.

62. Anjou-Lindskog E, Broman L, Broman M, Holmgren A. Effects of oxygen on central haemodynamics and VA/Q distribution after coronary bypass surgery. Acta Anaesthesiol Scand 1983;27(5):378-84.

63. Yende S, Quasney MW, Tolley EA, Wunderink RG. Clinical relevance of angiotensin-converting enzyme gene polymorphisms to predict risk of mechanical ventilation after coronary artery bypass graft surgery. Crit Care Med 2004;32(4):922-7.

64. Zabeeda D, Gefen R, Medalion B, Khazin V, Shachner A, Ezri T. The effect of high-frequency ventilation of the lungs on postbypass oxygenation: a comparison 
with other ventilation methods applied during cardiopulmonary bypass. J

Cardiothorac Vasc Anesth 2003;17(1):40-4.

65. Roediger L, Larbuisson R, Senard M, Hubert B, Damas P, Lamy M. New anesthetic and resuscitation techniques in adult cardiac surgery. Rev Med Liege 2004;59(1):35-45.

66. Hirai S, Hamanaka Y, Mitsui N, Morifuji K, Sutoh M. Clinical evaluation of risk factors for respiratory failure after coronary artery bypass grafting with cardiopulmonary bypass. Kyobu Geka 2004;57(9):851-5.

67. Konstantakos AK, Lee JH. Optimizing timing of early extubation in coronary artery bypass surgery patients. Ann Thorac Surg 2000;69(6):1842-5.

68. Calzia E, Koch M, Stahl W, Radermacher P, Brinkmann A. Stress response during weaning after cardiac surgery. Br J Anaesth 2001;87(3):490-3.

69. Kollef MH, Wragge T, Pasque C. Determinants of mortality and multiorgan dysfunction in cardiac surgery patients requiring prolonged mechanical ventilation. Chest 1995;107(5):1395-401.

70. Chong CF, Li YC, Wang TL, Chang H. Stratification of adverse outcomes by preoperative risk factors in coronary artery bypass graft patients: an artificial neural network prediction model. AMIA Annu Symp Proc 2003:160-4.

71. Dias FS, Milius G, Posenato AA, Palombini DV, Bodanese LC, Petracco JB. Prolonged mechanical ventilation following heart surgery. Arq Bras Cardiol 1992;59(4):269-73.

72. Saldias F, Castellon JM, Garayar B, Blacutt M. Predictor indices of early extubation in mechanical ventilation in patients treated with heart surgery. Rev Med Chil 1996;124(8):959-66.

73. Meade MO, Guyatt GH, Cook DJ. Weaning from mechanical ventilation: the evidence from clinical research. Respir Care 2001;46(12):1408-15; discussion 1415-7.

74. Epstein CD, El-Mokadem N, Peerless JR. Weaning older patients from long-term mechanical ventilation: a pilot study. Am J Crit Care 2002;11(4):369-77.

75. De Backer D, El Haddad P, Preiser JC, Vincent JL. Hemodynamic responses to successful weaning from mechanical ventilation after cardiovascular surgery. Intensive Care Med 2000;26(9):1201-6.

76. Doering LV, Esmailian F, Imperial-Perez F, Monsein S. Determinants of intensive care unit length of stay after coronary artery bypass graft surgery. Heart Lung 2001;30(1):9-17.

77. Paulissian R, Salem MR, Joseph NJ, Braverman B, Cohen HC, Crystal GJ, et al. Hemodynamic responses to endotracheal extubation after coronary artery bypass grafting. Anesth Analg 1991;73(1):10-5.

78. Hudson RJ, Thomson IR, Henderson BT, Singh K, Harding G, Peterson DJ. Validation of fentanyl pharmacokinetics in patients undergoing coronary artery bypass grafting. Can J Anaesth 2002;49(4):388-92.

79. Albage A, van der Linden J, Bengtsson L, Lindblom D, Kenneback G, Berglund H. Elevations in antidiuretic hormone and aldosterone as possible causes of fluid retention in the Maze procedure. Ann Thorac Surg 2001;72(1):58-64.

80. Boldt J, Hempelmann G. Hemodynamic effects of enoximone--comparative studies of heart surgery patients. Z Kardiol 1991;80 Suppl 4:41-6. 
81. Cote D, Martin R, Tetrault JP. Haemodynamic interactions of muscle relaxants and sufentanil in coronary artery surgery. Can J Anaesth 1991;38(3):324-9.

82. Weiskopf RB, Viele MK, Feiner J, Kelley S, Lieberman J, Noorani M, et al. Human cardiovascular and metabolic response to acute, severe isovolemic anemia. Jama 1998;279(3):217-21.

83. Wolfel EE, Selland MA, Cymerman A, Brooks GA, Butterfield GE, Mazzeo RS, et al. O2 extraction maintains $\mathrm{O} 2$ uptake during submaximal exercise with betaadrenergic blockade at 4,300 m. J Appl Physiol 1998;85(3):1092-102.

84. Frazier SK, Moser DK, Stone KS. Cardiac power output during transition from mechanical to spontaneous ventilation in canines. J Cardiovasc Nurs 2001;15(2):2332.

85. Jubran A, Mathru M, Dries D, Tobin MJ. Continuous recordings of mixed venous oxygen saturation during weaning from mechanical ventilation and the ramifications thereof. Am J Respir Crit Care Med 1998;158(6):1763-9.

86. Cason CL, DeSalvo SK, Ray WT. Changes in oxygen saturation during weaning from short-term ventilator support after coronary artery bypass graft surgery. Heart Lung 1994;23(5):368-75.

87. Kirkeby-Garstad I, Skogvoll E, Sellevold FM. Mixed venous oxygen saturation during mobilization after cardiac surgery: are reflectance oximetry catheters reliable? Acta Anaesthesiol Scand 2000;44(9):1103-8.

88. Kirkeby-Garstad I, Stenseth R, Sellevold OF. Post-operative myocardial dysfunction does not affect the physiological response to early mobilization after coronary artery bypass grafting. Acta Anaesthesiol Scand 2005;49(9):1241-7.

89. Estenssoro E, Gonzalez F, Laffaire E, Canales H, Saenz G, Reina R, et al. Shock on admission day is the best predictor of prolonged mechanical ventilation in the ICU. Chest 2005;127(2):598-603.

90. Ely EW, Meade MO, Haponik EF, Kollef MH, Cook DJ, Guyatt GH, et al. Mechanical ventilator weaning protocols driven by nonphysician health-care professionals: evidence-based clinical practice guidelines. Chest 2001;120(6 Suppl):454S-63S.

91. Baraka AS, Haroun-Bizri S, Shabb BR, Khoury SS, Chehab IR, Jalbout MI. Haemodynamic and EKG changes in patients undergoing minimally invasive direct coronary artery bypass. Middle East J Anesthesiol 2002;16(4):387-96.

92. Reich DL, Bennett-Guerrero E, Bodian CA, Hossain S, Winfree W, Krol M. Intraoperative tachycardia and hypertension are independently associated with adverse outcome in noncardiac surgery of long duration. Anesth Analg 2002;95(2):273-7.

93. Lieberman RW, Orkin FK, Jobes DR, Schwartz AJ. Hemodynamic predictors of myocardial ischemia during halothane anesthesia for coronary-artery revascularization. Anesthesiology 1983;59(1):36-41.

94. Hibbard MD, Holmes DR, Jr., Bailey KR, Reeder GS, Bresnahan JF, Gersh BJ. Percutaneous transluminal coronary angioplasty in patients with cardiogenic shock. J Am Coll Cardiol 1992;19(3):639-46.

95. Poeze M, Ramsay G, Greve JW, Singer M. Prediction of postoperative cardiac surgical morbidity and organ failure within 4 hours of intensive care unit admission using esophageal Doppler ultrasonography. Crit Care Med 1999;27(7):1288-94. 
96. David TE, Puschmann R, Ivanov J, Bos J, Armstrong S, Feindel CM, et al. Aortic valve replacement with stentless and stented porcine valves: a case-match study. J Thorac Cardiovasc Surg 1998;116(2):236-41.

97. Givertz MM, Hare JM, Loh E, Gauthier DF, Colucci WS. Effect of bolus milrinone on hemodynamic variables and pulmonary vascular resistance in patients with severe left ventricular dysfunction: a rapid test for reversibility of pulmonary hypertension. J Am Coll Cardiol 1996;28(7):1775-80.

98. Gavazzi A, Ghio S, Scelsi L, Campana C, Klersy C, Serio A, et al. Response of the right ventricle to acute pulmonary vasodilation predicts the outcome in patients with advanced heart failure and pulmonary hypertension. Am Heart J 2003;145(2):310-6.

99. Abalos A, Leibowitz AB, Distefano D, Halpern N, Iberti TJ. Myocardial ischemia during the weaning period. Am J Crit Care 1992;1(3):32-6.

100. Quatrini U, Mazzone M. Studies on the cardiovascular response to hypoxia. Arch Fisiol 1973;70(3-4):271-82.

101. Mehta S, Jay GD, Woolard RH, Hipona RA, Connolly EM, Cimini DM, et al. Randomized, prospective trial of bilevel versus continuous positive airway pressure in acute pulmonary edema. Crit Care Med 1997;25(4):620-8.

102. Combes A, Costa MA, Trouillet JL, Baudot J, Mokhtari M, Gibert C, et al. Morbidity, mortality, and quality-of-life outcomes of patients requiring $>$ or $=14$ days of mechanical ventilation. Crit Care Med 2003;31(5):1373-81.

103. Karakurt S, Fanfulla F, Nava S. Is it safe for patients with chronic hypercapnic respiratory failure undergoing home noninvasive ventilation to discontinue ventilation briefly? Chest 2001;119(5):1379-86.

104. Fullerton DA, Kirson LE, St Cyr JA, Albert JD, Whitman GJ. The influence of respiratory acid-base status on adult pulmonary vascular resistance before and after cardiopulmonary bypass. Chest 1993;103(4):1091-5.

105. Dryden CM, Smith DC, McLintic AJ, Pace NA. The effect of preoperative betablocker therapy on cardiovascular responses to weaning from mechanical ventilation and extubation after coronary artery bypass grafting. J Cardiothorac Vasc Anesth 1993;7(5):547-50.

106. Bueno R, Resende AC, Melo R, Neto VA, Stolf NA. Effects of hypertonic salinedextran solution in cardiac valve surgery with cardiopulmonary bypass. Ann Thorac Surg 2004;77(2):604-11.

107. Schmidt H, Rohr D, Bauer H, Bohrer H, Motsch J, Martin E. Changes in intrathoracic fluid volumes during weaning from mechanical ventilation in patients after coronary artery bypass grafting. J Crit Care 1997;12(1):22-7.

108. Meade M, Guyatt G, Griffith L, Booker L, Randall J, Cook DJ. Introduction to a series of systematic reviews of weaning from mechanical ventilation. Chest 2001;120(6 Suppl):396S-9S.

109. Bertolissi M, Bassi F, Cecotti R, Capelli C, Giordano F. Pruritus: a useful sign for predicting the haemodynamic changes that occur following administration of vancomycin. Crit Care 2002;6(3):234-9.

110. Krieger BP, Isber J, Breitenbucher A, Throop G, Ershowsky P. Serial measurements of the rapid-shallow-breathing index as a predictor of weaning outcome in elderly medical patients. Chest 1997;112(4):1029-34. 
111. Pappagallo M, Abbasi S, Bhutani VK. Respiratory and systemic effects of inhaled dexamethasone on ventilator dependant preterm infants at risk for bronchopulmonary dysplasia. Indian J Pediatr 1998;65(2):273-82.

112. Song JM, Kang DH, Song JK, Jeong YH, Lee CW, Hong MK, et al. Outcome of significant functional tricuspid regurgitation after percutaneous mitral valvuloplasty. Am Heart J 2003;145(2):371-6.

113. Palacios IF, Sanchez PL, Harrell LC, Weyman AE, Block PC. Which patients benefit from percutaneous mitral balloon valvuloplasty? Prevalvuloplasty and postvalvuloplasty variables that predict long-term outcome. Circulation 2002;105(12):1465-71.

114. Dighero H, Zepeda F, Sepulveda P, Soto JR, Aranda W. Percutaneous mitral balloon valvotomy: six-year follow-up. J Invasive Cardiol 2001;13(12):795-9.

115. Murillo H, Ayala F, Badui E, Almazan A, Solorio S, Enciso R, et al. Percutaneous mitral commissurotomy using Inoue's balloon during pregnancy. Arch Inst Cardiol Mex 1996;66(4):350-5.

116. Cardaioli P, Zennaro M, Ramondo A, Isabella G, Razzolini R, Marchiori MC, et al. Regression of pulmonary hypertension in mitral stenosis: an echo-hemodynamic study in patients who underwent mitral balloon valvuloplasty. G Ital Cardiol 1994;24(4):381-9.

117. Greenough A. Respiratory support techniques for prematurely born infants: new advances and perspectives. Acta Paediatr Taiwan 2001;42(4):201-6.

118. Patole S, Lee J, Whitehall J. Adenosine infusion in the management of a micropremi neonate with pulmonary hypertension. Indian Pediatr 1998;35(12):1221-4.

119. Schulze-Neick I, Werner H, Penny DJ, Alexi-Meskishvili V, Lange PE. Acute ventilatory restriction in children after weaning off inhaled nitric oxide: relation to rebound pulmonary hypertension. Intensive Care Med 1999;25(1):76-80.

120. Maxey TS, Smith CD, Kern JA, Tribble CG, Jones DR, Kron IL, et al. Beneficial effects of inhaled nitric oxide in adult cardiac surgical patients. Ann Thorac Surg 2002;73(2):529-32; discussion 532-3.

121. Chhabra SK, De S. Cardiovascular autonomic neuropathy in chronic obstructive pulmonary disease. Respir Med 2005;99(1):126-33.

122. Georgopoulos D, Mitrouska I, Markopoulou K, Patakas D, Anthonisen NR. Effects of breathing patterns on mechanically ventilated patients with chronic obstructive pulmonary disease and dynamic hyperinflation. Intensive Care Med 1995;21(11):880-6.

123. Anand IS, Chandrashekhar Y, Ferrari R, Sarma R, Guleria R, Jindal SK, et al. Pathogenesis of congestive state in chronic obstructive pulmonary disease. Studies of body water and sodium, renal function, hemodynamics, and plasma hormones during edema and after recovery. Circulation 1992;86(1):12-21.

124. Butler J, Schrijen F, Henriquez A, Polu JM, Albert RK. Cause of the raised wedge pressure on exercise in chronic obstructive pulmonary disease. Am Rev Respir Dis 1988;138(2):350-4.

125. Gel'tser BI, Frisman MV. Assessment of the quality of life of cardiological patients: current approach. Klin Med (Mosk) 2002;80(9):4-9. 
126. Kavarana MN, Pessin-Minsley MS, Urtecho J, Catanese KA, Flannery M, Oz MC, et al. Right ventricular dysfunction and organ failure in left ventricular assist device recipients: a continuing problem. Ann Thorac Surg 2002;73(3):745-50.

127. Binkley PF, Nunziata E, Haas GJ, Starling RC, Leier CV, Cody RJ. Dissociation between ACE activity and autonomic response to ACE inhibition in patients with heart failure. Am Heart J 2000;140(1):34-42.

128. Guerin AP, Adda H, London GM, Marchais SJ. Cardiovascular disease in renal failure. Minerva Urol Nefrol 2004;56(3):279-88.

129. Alpert MA. Cardiac performance and morphology in end-stage renal disease. Am J Med Sci 2003;325(4):168-78.

130. London GM. Left ventricular alterations and end-stage renal disease. Nephrol Dial Transplant 2002;17 Suppl 1:29-36.

131. Schafer GE, Rehbein C, Stiegler T, Hampl H. Renal anemia and its hemodynamic response--findings invasively determined over a period of 20 years. Clin Nephrol 2002;58 Suppl 1:S52-7.

132. Amabile N, Guerin AP, Leroyer A, Mallat Z, Nguyen C, Boddaert J, et al. Circulating endothelial microparticles are associated with vascular dysfunction in patients with end-stage renal failure. J Am Soc Nephrol 2005;16(11):3381-8.

133. Rundback JH, Murphy TP, Cooper C, Weintraub JL. Chronic renal ischemia: pathophysiologic mechanisms of cardiovascular and renal disease. J Vasc Interv Radiol 2002;13(11):1085-92.

134. Zhou X, Matavelli L, Frohlich ED. Uric acid: its relationship to renal hemodynamics and the renal renin-angiotensin system. Curr Hypertens Rep 2006;8(2):120-4.

135. Kotik L, Fabian J, Horak K, Firt P. Haemodynamic changes immediately following myocardial revascularization. Cor Vasa 1983;25(2):100-7.

136. Dries DJ, Kumar P, Mathru M, Mayer R, Zecca A, Rao TL, et al. Hemodynamic effects of pressure support ventilation in cardiac surgery patients. Am Surg 1991;57(2):122-5.

137. Lehot JJ, Arvieux CC, Viale JP, Foex P. Myocardial ischemia and anesthesia. Ann Fr Anesth Reanim 1995;14(2):176-97.

138. Gardner SC, Grunwald GK, Rumsfeld JS, Mackenzie T, Gao D, Perlin JB, et al. Risk factors for intermediate-term survival after coronary artery bypass grafting. Ann Thorac Surg 2001;72(6):2033-7.

139. Cox CE, Carson SS, Holmes GM, Howard A, Carey TS. Increase in tracheostomy for prolonged mechanical ventilation in North Carolina, 1993-2002. Crit Care Med 2004;32(11):2219-26.

140. Epstein SK, Ciubotaru RL, Wong JB. Effect of failed extubation on the outcome of mechanical ventilation. Chest 1997;112(1):186-92.

141. Epstein SK, Nevins ML, Chung J. Effect of unplanned extubation on outcome of mechanical ventilation. Am J Respir Crit Care Med 2000;161(6):1912-6.

142. Epstein SK, Vuong V. Lack of influence of gender on outcomes of mechanically ventilated medical ICU patients. Chest 1999;116(3):732-9.

143. Nevins ML, Epstein SK. Predictors of outcome for patients with COPD requiring invasive mechanical ventilation. Chest 2001;119(6):1840-9. 
144. Jesurum JT, Alexander WA, Anderson JJ, Houston S. Fast Track recovery after aortocoronary bypass surgery: early extubation and intensive care unit transfer. Semin Perioper Nurs 1996;5(1):12-22.

145. Cohen AJ, Katz MG, Frenkel G, Medalion B, Geva D, Schachner A. Morbid Results of Prolonged Intubation After Coronary Artery Bypass Surgery. Chest 2000;118:1724-1731.

146. Arom KV, Emery RW, Petersen RJ, Schwartz M. Cost-effectiveness and predictors of early extubation. Ann Thorac Surg 1995;60(1):127-32.

147. Alexander W. Nursing challenge: early extubation after cardiac surgery. Todays OR Nurse 1994;16(6):47-9.

148. Cohen AJ, Katz MG, Frenkel G, Medalion B, Geva D, Schachner A. Morbid results of prolonged intubation after coronary artery bypass surgery. Chest 2000;118(6):1724-31.

149. Doering LV. Relationship of age, sex, and procedure type to extubation outcome after heart surgery. Heart Lung 1997;26(6):439-47.

150. Djaiani GN, Ali M, Heinrich L, Bruce J, Carroll J, Karski J, et al. Ultra-fast-track anesthetic technique facilitates operating room extubation in patients undergoing off-pump coronary revascularization surgery. J Cardiothorac Vasc Anesth 2001;15(2):152-7.

151. Gluck EH. Predicting eventual success or failure to wean in patients receiving longterm mechanical ventilation. Chest 1996;110(4):1018-24.

152. Souza MHL, Elias DO. Weaning from cardiopulmonary bypass. Indian Journal of Extracorporeal Technology 1998;6(2).

153. Epstein SK. Predicting extubation failure: is it in (on) the cards? Chest 2001;120(4):1061-3.

154. Leitch EA, Moran JL, Grealy B. Weaning and extubation in the intensive care unit. Clinical or index-driven approach? Intensive Care Med 1996;22(8):752-9.

155. Celkan MA, Ustunsoy H, Daglar B, Kazaz H, Kocoglu H. Readmission and mortality in patients undergoing off-pump coronary artery bypass surgery with fasttrack recovery protocol. Heart Vessels 2005;20(6):251-5.

156. Myles PS, McIlroy D. Fast-track cardiac anesthesia: choice of anesthetic agents and techniques. Semin Cardiothorac Vasc Anesth 2005;9(1):5-16.

157. Watanabe Y, Kosaka M, Kusume Y, Suga T, Hatakenaka T, Omachi N, et al. Fasttrack cardiac anesthesia and perioperative management appropriate for early rehabilitation after coronary artery bypass graft (CABG) surgery. Masui 2004;53(8):898-902.

158. Murphy GS, Szokol JW, Marymont JH, Vender JS, Avram MJ, Rosengart TK, et al. Recovery of neuromuscular function after cardiac surgery: pancuronium versus rocuronium. Anesth Analg 2003;96(5):1301-7.

159. Bowler I, Djaiani G, Abel R, Pugh S, Dunne J, Hall J. A combination of intrathecal morphine and remifentanil anesthesia for fast-track cardiac anesthesia and surgery. J Cardiothorac Vasc Anesth 2002;16(6):709-14.

160. Reis J, Mota JC, Ponce P, Costa-Pereira A, Guerreiro M. Early extubation does not increase complication rates after coronary artery bypass graft surgery with cardiopulmonary bypass. Eur J Cardiothorac Surg 2002;21(6):1026-30. 
161. Cheng DC, Newman MF, Duke P, Wong DT, Finegan B, Howie M, et al. The efficacy and resource utilization of remifentanil and fentanyl in fast-track coronary artery bypass graft surgery: a prospective randomized, double-blinded controlled, multi-center trial. Anesth Analg 2001;92(5):1094-102.

162. Howie MB, Cheng D, Newman MF, Pierce ET, Hogue C, Hillel Z, et al. A randomized double-blinded multicenter comparison of remifentanil versus fentanyl when combined with isoflurane/propofol for early extubation in coronary artery bypass graft surgery. Anesth Analg 2001;92(5):1084-93.

163. Montes FR, Sanchez SI, Giraldo JC, Rincon JD, Rincon IE, Vanegas MV, et al. The lack of benefit of tracheal extubation in the operating room after coronary artery bypass surgery. Anesth Analg 2000;91(4):776-80.

164. Royston D. Patient selection and anesthetic management for early extubation and hospital discharge: CABG. J Cardiothorac Vasc Anesth 1998;12(6 Suppl 2):11-9.

165. Silbert BS, Santamaria JD, O'Brien JL, Blyth CM, Kelly WJ, Molnar RR. Early extubation following coronary artery bypass surgery: a prospective randomized controlled trial. The Fast Track Cardiac Care Team. Chest 1998;113(6):1481-8.

166. Liu LL, Gropper MA. Respiratory and Hemodynamic Management After Cardiac Surgery. Curr Treat Options Cardiovasc Med 2002;4(2):161-169.

167. Vedrinne C, Bastien O, De Varax R, Blanc P, Durand PG, Du Gres B, et al. Predictive factors for usefulness of fiberoptic pulmonary artery catheter for continuous oxygen saturation in mixed venous blood monitoring in cardiac surgery. Anesth Analg 1997;85(1):2-10.

168. Van den Berghe G, Wouters P, Weekers F, al. e. Intensive insulin therapy in the critically ill patients. New England Journal of Medicine 2001;345(1359-1367).

169. Lemaire F. Respiratory muscle fatigue and acute respiratory failure, role during weaning failure. Monaldi Arch Chest Dis 1993;48(5):494-7.

170. Lemaire F. Difficult weaning. Intensive Care Med 1993;19 Suppl 2:S69-73.

171. Frazier SK, Stone KS, Schertel ER, Moser DK, Pratt JW. A comparison of hemodynamic changes during the transition from mechanical ventilation to T-piece, pressure support, and continuous positive airway pressure in canines. Biol Res Nurs 2000;1(4):253-64.

172. Troche $G$, Moine $P$. Is the duration of mechanical ventilation predictable? Chest 1997;112(3):745-51.

173. Capdevila XJ, Perrigault PF, Perey PJ, Roustan JP, d'Athis F. Occlusion pressure and its ratio to maximum inspiratory pressure are useful predictors for successful extubation following T-piece weaning trial. Chest 1995;108(2):482-9.

174. Kollef MH, Shapiro SD, Silver P, St John RE, Prentice D, Sauer S, et al. A randomized, controlled trial of protocol-directed versus physician-directed weaning from mechanical ventilation. Crit Care Med 1997;25(4):567-74.

175. De Souza RL, de Carvalho WB. Preliminary study about the utility of gastric tonometry during the weaning from mechanical ventilation. Rev Assoc Med Bras 2002;48(1):66-72.

176. Hurst JM, Branson RD, Davis K, Jr., Barrette RR. Cardiopulmonary effects of pressure support ventilation. Arch Surg 1989;124(9):1067-70. 
177. Savino JS, Ley C, Boisvert D, Friedman A, Mathew J, Koch C, et al. Practice pattern variability for myocardial revascularization: impact on resource use across 24 centers. J Cardiothorac Vasc Anesth 2002;16(2):149-56.

178. Brum G, Melo R, Valenca J, Vizcaino J, Monteiro F, Cardim P, et al. Prolonged mechanical ventilation in a respiratory ICU. Rev Port Pneumol 2003;9(5 Suppl):278.

179. Chao DC, Scheinhorn DJ, Stearn-Hassenpflug M. Patient-ventilator trigger asynchrony in prolonged mechanical ventilation. Chest 1997;112(6):1592-9.

180. Chailleux E, Fauroux B, Binet F, Dautzenberg B, Polu JM. Predictors of survival in patients receiving domiciliary oxygen therapy or mechanical ventilation. A 10-year analysis of ANTADIR Observatory. Chest 1996;109(3):741-9.

181. Shang M, Wang C, Dai H, Yang Y, Jiang C. Changes in respiratory and circulatory functions during sequential invasive-noninvasive mechanical ventilation. Chin Med J (Engl) 2003;116(8):1253-6.

182. Shang $\mathrm{M}$, Wang $\mathrm{C}$, Dai $\mathrm{H}$. Changes in respiratory and circulatory function during sequential invasive-noninvasive mechanical ventilation. Zhonghua Jie $\mathrm{He} \mathrm{He} \mathrm{Hu} \mathrm{Xi}$ Za Zhi 2001;24(8):487-9.

183. Epstein SK. Weaning from mechanical ventilation. Respir Care 2002;47(4):454-66; discussion 466-8.

184. Smyrnios NA, Connolly A, Wilson MM, Curley FJ, French CT, Heard SO, et al. Effects of a multifaceted, multidisciplinary, hospital-wide quality improvement program on weaning from mechanical ventilation. Crit Care Med 2002;30(6):122430 .

185. Gordis L. Epidemiology. Third ed. Maryland: Elsevier; 2004.

186. Pedhazur EJ. Measurement, Design, and Analysis; An Integrated Approach. First ed. New Jersey: Lawrence Erlbaum Associates Publishers; 1991.

187. Rosner B. Fundamentals of Biostatistics. Fifth ed. United States: Duxbury; 2000.

188. Saunder R. An introduction to biostatistics. New Delhi: Prentice Hall of India; 1996.

189. Athanasiou T, Al-Ruzzeh S, Del Stanbridge R, Casula RP, Glenville BE, Amrani M. Is the female gender an independent predictor of adverse outcome after off-pump coronary artery bypass grafting? Ann Thorac Surg 2003;75(4):1153-60.

190. Parlow JL, Ahn R, Milne B. Obesity is a risk factor for failure of "fast track" extubation following coronary artery bypass surgery. Can J Anaesth 2006;53(3):288-94. 
APPENDIX A: UNIVERSITY OF TENNESSEE HEALTH SCIENCE CENTER IRB APPROVAL LETTER 


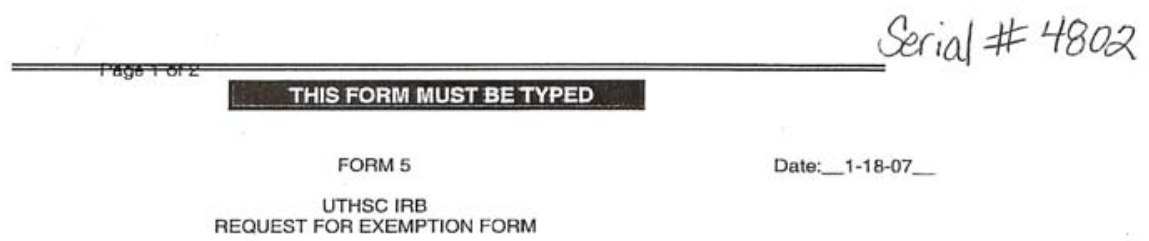

PROPOSAL TITLE:__ Cardio Pulmonary Indicators in Prediction of Dysfunctional Ventilator Weaning Response after Coronary Artery Bypass Graft surgery _

INVESTIGATOR: ANNAPOORNA MARY

DEPARTMENT \& SCHOOL: _ Graduate College - NURSING

TELEPHONE \#: _ $901-680-0137 \_$FAX \#: _901-448-4121

ADDRESS:_ UTHSC College of Nursing

877 Madison Avenue

Room 631

Memphis, TN 38163

E-MAIL: _amary@utmem.edu

FUNDING SOURCE (SPONSOR):

STUDY SITE(S):

St. Francis Hospital, Memphis TN

(Answer all items, attach separate sheets as necessary) PURPOSE OF THE STUDY:

Dysfunctional ventilator weaning response (DVWR) is a major problem among postoperative coronary artery bypass graft surgery $(\mathrm{CABG})$ patients. Although majority of the patients are extubated within six to eight hours, 20 to $40 \%$ of patients remain intubated twelve hours after surgery due to dysfunctional ventilator weaning response (DVWR) [1, 6-25].

Dysfunctional ventilator weaning response( DVWR ) is associated with increased morbidity, and mortality following CABG surgery $[2,15,22,44-46]$. The mortality related to DVWR is reported as 30 to $43 \%[20,21]$. It is resulting from many complications of DVWR such as acute respiratory distress syndrome 10 to $15 \%[20,21]$, multi organ dysfunction syndrome 15 to $19 \%$, deep vein thrombosis 10 to $12 \%$ [ $41-43]$ and ventilator associated pneumonia 30 to $50 \%[47,48]$. The other health care problems resulting from DVWR are increased ICU length of stay and ICU cost [8, 15, $49,50]$. Early detection of DVWR is imperative to prevent the postoperative complication after CABG surgery. Finding the reliable predictive factors for DVWR could help in early detection of DVWR before the occurrence, may guide in planning the early treatment for DVWR, and may aid in preventing complications after CABG surgery. 
data. All personal information in the medical records that may reveal the identity of the

individuals will be removed after coding, and the anonymity of the data will be maintained.

EXEMPTION CATEGORY: Indicate the appropriate exemption category.

a. The study does not involve "research" as defined in the regulations.

b. The study does not use "human subjects" as defined in the regulations.

(i) All data or specimens are from deceased persons.

(ii) No data or specimens can be linked to identifiable individuals.

c. The study qualifies for exempt status under 45CFR46.101(b). (Indicate category (b)(1) or (b)(2), etc.)

INFORMED CONSENT: Informed consent is generally required for an exempt study unless it cannot practicably be carried out without a waiver of consent. Please check the appropriate box.

a. The study cannot practicably be carried out without a waiver of consent.

b. The study can be undertaken even if informed consent is required.

If line (a) is checked, briefly explain below why the study cannot practicably be carried out without a waiver of consent. If the study involves the use of protected health information, also complete Form 8 to request the use of protected health information without subject authorization.

A waiver of all the consent requirements is requested because this research doesn't pose any risk to the individuals listed in the medical records. All identifying information will be coded and removed to protect their rights and privacy. The researchers will access pre-existing medical records through EPF system after obtaining the security PIN form the mid south private hospital. The hospital HIPPA privacy policy will be maintained in handling the data. It would be impossible to re-contact former patients to secure their consent for a retrospective medical record review; especially this study involves reviewing more than a thousand medical records to select sample size of 300
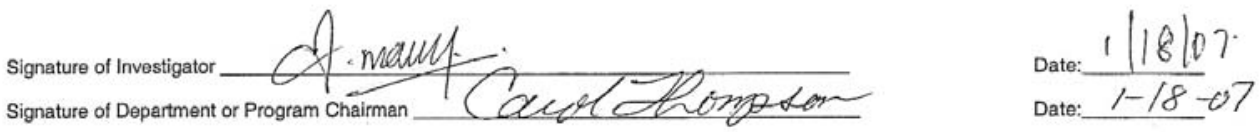

Typewritten Name of Department or Program Chairman

Carol Thompson, $\mathrm{PhD}$ 
Principal Investigator: ___ Annapoorna Mary
Brief Project Title: $\quad$ Cardio Pulmonary Indicators in Prediction of Dysfunctional

Ventilator Weaning Response After Coronary Artery Bypass Graft Surgery

IRB ACTION:

Approved ___ ___ Approved w/proviso(s)

Referred For Board Review

COMMENTS: This study qualifies for exempt status under 45CFR46.101(b)(4). Informed consent is waived in accord with 45CFR46.116(d).

The study is also approved for waiver of the HIPAA authorization for the conduct of the study itself. The waiver applies to the medical records of patients undergoing CABG at St. Francis Hospital, Memphis between January 2002 and February 28, 2006.

Consent Required: No: $\mathcal{C}$ Yes: Not Applicable: Written

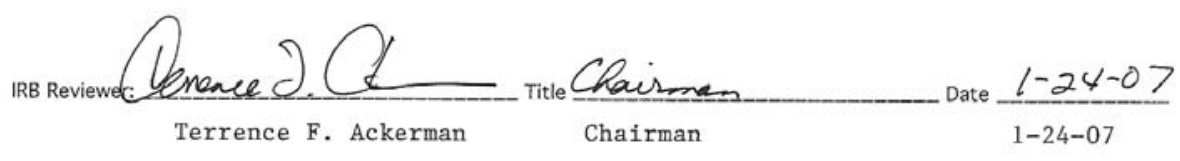


APPENDIX B: ST. FRANCIS HOSPITAL, MEMPHIS, IRB APPROVAL LETTER 


\section{Saint Francis Hospital}

Memphis

5959 Park Avenue

Memphis, TN 38119.5198

Tel 901.765 .1000

hitp://www.tenetheolth.com

February 3, 2007

Ms Annapoorna Mary

1528 Mount Moriah Road

Memphis, TN 38117

RE Cardio Pulmonary Indications in Prediction of Dysfunctional Ventilator Weaning Response after Coronary Artery Bypass Graft Surgery

Dear Ms Mary,

I have reviewed this proposal and find it has scientific merit as well as meeting the requirements for Expedited Review as set down in the Saint Francis Hospital Institutional Review policies.

You are granted permission to initiate this investigation. This protocol will be mentioned as an information item at our next IRB meeting in April. Please ensure that you are in compliance with all hospital policies concerning privacy are confidentiality.

This protocol requires annual reports on beginning February 2008 and a final report when completed. Thank you for your support of our research program.

If you have any questions please feel free to contact me through our Medical Staff Office or our IRB Administrator at 7651393.

Yours very truly

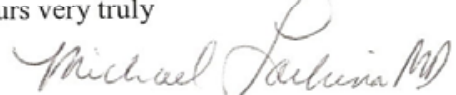

F Michael Lachina, MD, CMO

Chairman, Institutional Review Board

Saint Francis Hospital. 


\section{VITA}

Annapoorna Mary was born in south India on April 29,1963. She obtained a Diploma in General Nursing and Certification in Midwifery from St. Ann's School of Nursing, affiliated with Tamil Nadu State Nursing Council, in 1984. She graduated from Madras Medical College (affiliated with Tamil Nadu Dr. MGR Medical University) with a Bachelor of Science in Nursing in 1999. She received a Master of Science in Nursing, specializing in Medical Surgical Nursing and Critical Care Nursing, from Sri.

Ramachandra Medical College and Research Institute, Madras, in 2002. She has functioned as a nurse for the past 23 years in various vital areas of healthcare such as Emergency Department, Medical Intensive Care Unit, Neonatal Intensive Care Unit, Cardiovascular Intensive Care Unit as a clinician, care provider, administrator, educator, and researcher. In addition, she has experience working in healthcare involving various levels such as rural, urban, corporate, governmental, nongovernmental, and international. She investigated the "Effectiveness of Standard Endotracheal Suctioning Technique" in 2001, which was published in Trained Nurses Association of India Journal in 2005. She received certification in CCRN in 2003. She also received certification in various qualifying exams such as CGFNS, CPR, ACLS, PALS, Trauma Care, and Chemotherapy Provider. She is an active member of TNAI (India), AACCN, AHA, and Sigma Theta Tau. 